\title{
2.DATA REPORT: MAJOR AND TRACE ELEMENT GEOCHEMISTRY OF SHIPBOARD SAMPLES FROM SITE 957, TAG HYDROTHERMAL FIELD, MID-ATLANTIC RIDGE ${ }^{1}$
}

\author{
Mark D. Hannington, ${ }^{2}$ Peter M. Herzig, ${ }^{3}$ Sven Petersen,${ }^{3}$ D. Conrad Gregoire,,${ }^{2}$ Peter Belanger ${ }^{2}$
}

\section{INTRODUCTION}

Representative samples of drill core were collected from each of the five main areas drilled on the TAG (Trans-Atlantic Geotraverse) mound during Leg 158 (Humphris, Herzig, Miller, et al., 1996). In this report, we present the results of chemical analyses of 66 samples previously analyzed for $\mathrm{Cu}, \mathrm{Fe}, \mathrm{Zn}, \mathrm{Pb}, \mathrm{Ag}$, and $\mathrm{Cd}$ by atomic absorption during Leg 158. Data are presented for an additional 38 elements plus total sulfur, loss on ignition, and the rare earth elements by a combination of optical emission spectrometry (ICP-ES), mass spectrometry (ICP-MS), and neutron activation (INAA). These data are discussed in detail in other chapters in this volume.

\section{METHODS}

\section{Bulk Samples}

Sulfide-bearing samples were collected for shipboard chemical analyses from each section of core. Detailed descriptions of the sampling methods and sample preparation were previously reported by the Shipboard Scientific Party (Humphris, Herzig, Miller, et al., 1996). Nine different lithologies from the five areas are represented in the bulk samples, including (1) cherty sulfide breccias and related surface materials, (2) red and gray cherts, (3) porous massive pyrite \pm sphalerite, (4) massive granular pyrite, (5) massive pyrite breccias, (6) pyrite-anhydrite breccias, (7) pyrite-silica breccias, (8) silicified wallrock breccias, (9) chloritized basalt breccias. These sample types are described in detail in Humphris, Herzig, Miller, et al. (1996) and elsewhere in this volume.

All samples were crushed and pulverized to $<63 \mu \mathrm{m}$ in a hardened steel mill. This may have introduced a contamination of up to $75 \mathrm{ppm}$ $\mathrm{Cr}$ in some samples (cf. Hickson and Juras, 1986). From about $50 \mathrm{~cm}^{3}$ of material that was prepared, $1 \mathrm{~g}$ was taken for shipboard analyses, and the remainder was kept for more comprehensive shore-based analyses described here. The reported data include three duplicate analyses (laboratory duplicate) and three duplicate samples (sampling duplicates) for comparison of results. Three samples were prepared at different mesh sizes $(-63 \mu \mathrm{m}, 63-420 \mu \mathrm{m},+420 \mu \mathrm{m})$ to compare the grain-size distribution of different components.

Analysis of trace elements by inductively coupled plasma (ICP) spectrochemical techniques was based on the total dissolution of $1 \mathrm{~g}$ of sample using a mixed-acid digestion of nitric, hydrochloric, perchloric and hydrofluoric acids followed by a lithium metaborate fusion of any residual material. The dissolved samples and fusion melt were combined and diluted to a final volume of $100 \mathrm{~mL}$ in $10 \%$ hydrochloric acid. Analysis for the major elements by ICP was based on the fusion of $0.5 \mathrm{~g}$ of sample with a mixed lithium metaborate-tetraborate flux. The fusion melt was dissolved to give a final solution of $100 \mathrm{~mL}$ in $4 \%$ nitric acid. The dissolution method is modified from

${ }^{1}$ Herzig, P.M., Humphris, S.E., Miller, D.J., and Zierenberg, R.A. (Eds.), 1998 Proc. ODP, Sci. Results, 158: College Station, TX (Ocean Drilling Program).

${ }^{2}$ Geological Survey of Canada, 601 Booth Street, Ottawa, Ontario K1A 0E8, Canada. markh@gsc.emr.ca

${ }^{3}$ Institut für Mineralogie, TU Bergakademie Freiberg, Brennhausgasse 14, 09596 Freiberg, Federal Republic of Germany.
Bouvier (1991). The major elements and selected trace metals $(\mathrm{Cu}$, $\mathrm{Zn}, \mathrm{Ni}, \mathrm{Co}, \mathrm{V}, \mathrm{Ba}, \mathrm{Sr}, \mathrm{Be}, \mathrm{Sc}, \mathrm{Cr}$ ) were analyzed by emission spectrometry (ICP-ES). These data are listed in Tables 1 and 2. Total S was analyzed by combustion followed by infrared spectrophotometry (LECO). Loss on ignition (LOI) is determined by gravimetry at $900^{\circ} \mathrm{C}$. For low-level traces ( $\mathrm{Ag}, \mathrm{Pb}, \mathrm{Cd}, \mathrm{In}, \mathrm{Mo}, \mathrm{Tl}, \mathrm{Ga}, \mathrm{Rb}, \mathrm{Cs}, \mathrm{Nb}$, $\mathrm{Ta}, \mathrm{Zr}, \mathrm{Hf}, \mathrm{U}, \mathrm{Th}$ ) analysis was performed by mass spectrometry (ICP-MS). These data are listed in Table 3. Determination limits for each method were calculated from a large number of replicate analyses of reference materials but may vary with sample matrix. The limits indicated for each element in the tables take into account errors arising from all aspects of the analytical methods.

Analysis of trace elements by neutron activation was carried out on 1-g samples, irradiated by thermal neutrons in a high-flux reactor. Concentrations of Au, Ag, As, Sb, Fe, Zn, Mo, Co, Se, Hg, Sn, W, $\mathrm{Br}, \mathrm{Ir}, \mathrm{U}$ and $\mathrm{Th}$ were determined by gamma-ray spectrometry using a solid-state detector and are reported in Table 4. This method is particularly sensitive for trace metals such as $\mathrm{Au}, \mathrm{Ag}, \mathrm{As}, \mathrm{Sb}, \mathrm{Co}$, and $\mathrm{Se}$, however, certain elements such as $\mathrm{Ni}, \mathrm{Sn}, \mathrm{Ca}, \mathrm{Sr}$, and $\mathrm{Rb}$ are not well activated and have reduced sensitivity (e.g., Hannington and Gorton, 1991, and references therein). INAA results for $\mathrm{Ca}, \mathrm{Ba}, \mathrm{Na}$, $\mathrm{Cr}$, Cs, Hf, Ni, Rb, Sc, Sr, Ta, Th, and the REEs (La, Ce, Nd, Sm, Eu, $\mathrm{Tb}, \mathrm{Yb}, \mathrm{Lu}$ ) were at or below detection limits for this method in nearly all samples and are not reported. Several elements (Fe, Zn, Ag, Co, Mo, and U) were determined both by ICP and INAA with the same sensitivity, and both results are reported for comparison of the different methods.

Rare earth element concentrations (REE) were determined by ICP-MS, following the method used for trace elements, outlined above. These data are listed in Table 5.

\section{Mineral Separates}

Hand-picked concentrates of pyrite and chalcopyrite were prepared on board from intact drill core and analyzed separately by INAA, employing sample weights of 500-1000 mg. These data are listed in Table 6 . Samples of pyrite and chalcopyrite were collected from eight different lithologies, mainly in the TAG-1 area (chalcopyrite from section 957B-1R-2, sample 1, is from a near-surface sample in the TAG-2 area). The lithologies sampled include (1) massive granular pyrite, (2) massive pyrite breccias, (3) pyrite-anhydrite breccias, (4) pyrite-silica breccias, (5) pyrite-silica-anhydrite breccias, (6) silicified wallrock breccias, (7) chloritized basalt breccias, and (8) coarse anhydrite veins, which crosscut all of the other lithologies. Results for selected trace elements are reported in Table 2. The quality of the separations is indicated by the high $\mathrm{Fe}(33.8-53.6 \mathrm{wt} \%$ Fe for pyrite and $28.0-49.9 \mathrm{wt} \%$ $\mathrm{Fe}$ for chalcopyrite) and the uniformly low $\mathrm{Ca}$ contents of the samples.

\section{ACKNOWLEDGMENTS}

This research was carried out jointly by the Geological Survey of Canada (GSC) and by the Technical University Bergakademie Freiberg (TUBAF). ICP-ES and ICP-MS analyses were conducted in the laboratories of the GSC under the direction of D.C. Gregoire and

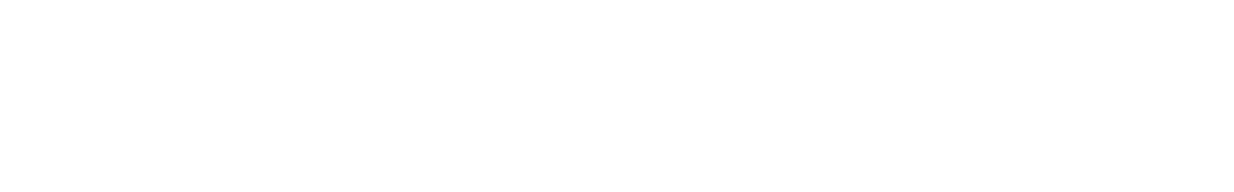


P. Belanger. INAA analyses were carried out by Activation Laboratories, Ancaster, Ontario, supported by a grant from the German Ocean Drilling Program to P.M. Herzig (TUBAF). This is GSC Contribution \#1996367.

\section{REFERENCES}

Bouvier, J.-L., 1991. Total dissolution method for rock samples. MRD/ACS, Geol. Surv. Can., Internal Tech. Rep.

Hannington, M.D., and Gorton, M.P., 1991. Analysis of sulfides for gold and associated trace metals by direct neutron activation with a low-flux reactor. Geostand. Newsl., 15:145-154.

Hickson, C.J., and Juras, S.J., 1986. Sample contamination by grinding. Can. Mineral., 24:585-589.

Humphris, S.E., Herzig, P.M., Miller, D.J., et al., 1996. Proc. ODP, Init. Repts., 158: College Station, TX (Ocean Drilling Program).

Date of initial receipt: 29 May 1996

Date of acceptance: 23 December 1996

Ms 158SR-203
Table 1. Major element composition of samples from Site 957.

\begin{tabular}{|c|c|c|c|c|}
\hline $\begin{array}{l}\text { Core, section, } \\
\text { interval }(\mathrm{cm})\end{array}$ & Piece & $\begin{array}{l}\text { Depth } \\
\text { (mbsf) }\end{array}$ & Area & Description \\
\hline $\begin{array}{l}158-957 \mathrm{~A}- \\
3 \mathrm{X}-1,4-8\end{array}$ & 1 & 10.00 & TAG-2 & Chert-sulfide breccia \\
\hline $\begin{array}{l}\text { 158-957B- } \\
1 \mathrm{R}-1,3-95 \\
1 \mathrm{R}-1,3-95 \\
1 \mathrm{R}-1,3-95 \\
1 \mathrm{R}-1,3-95 \\
4 \mathrm{R}-1,0-10\end{array}$ & $\begin{array}{c}-63 \\
63-420 \\
+420 \\
+420 \\
1\end{array}$ & $\begin{array}{r}0.00 \\
0.00 \\
0.00 \\
0.00 \\
19.90\end{array}$ & $\begin{array}{l}\text { TAG-2 } \\
\text { TAG-2 } \\
\text { TAG-2 } \\
\text { TAG-2 } \\
\text { TAG-2 }\end{array}$ & $\begin{array}{l}\text { Surface material } \\
\text { Surface material } \\
\text { Surface material } \\
\text { (Laboratory duplicate) } \\
\text { Altered basalt }\end{array}$ \\
\hline $\begin{array}{l}158-957 \mathrm{C}- \\
5 \mathrm{~N}-1,15-48 \\
5 \mathrm{~N}-1,15-48 \\
7 \mathrm{~N}-1,52-56 \\
7 \mathrm{~N}-2,63-69 \\
10 \mathrm{~N}-1,21-22 \\
11 \mathrm{~N}-1,58-61 \\
11 \mathrm{~N}-3,90-94 \\
12 \mathrm{~N}-1,53-59 \\
12 \mathrm{~N}-2,68-75 \\
12 \mathrm{~N}-3,86-100 \\
13 \mathrm{~N}-1,12-20 \\
14 \mathrm{~N}-1,33-41 \\
14 \mathrm{~N}-2,76.5-88 \\
15 \mathrm{~N}-1,36-40 \\
15 \mathrm{~N}-2,12-15 \\
15 \mathrm{~N}-3,132-150 \\
15 \mathrm{~N}-4,8-10 \\
16 \mathrm{~N}-1,118-125 \\
16 \mathrm{~N}-2,14-23 \\
16 \mathrm{~N}-2,94-95 \\
16 \mathrm{~N}-2,94-95\end{array}$ & $\begin{array}{c}3,5,6 \\
3,5,6 \\
6 \mathrm{~F} \\
1 \mathrm{E} \\
4 \\
3 \mathrm{D} \\
8 \mathrm{C} \\
6 \mathrm{~A} \\
9 \\
9 \\
4 \\
5 \\
6 \\
5 \\
1 \mathrm{~B} \\
11 \\
1 \\
15 \\
2 \\
9 \mathrm{~B} \\
9 \mathrm{~B}\end{array}$ & $\begin{array}{l}15.25 \\
15.25 \\
20.04 \\
21.45 \\
28.86 \\
31.18 \\
34.50 \\
35.45 \\
36.16 \\
36.94 \\
37.31 \\
40.53 \\
41.67 \\
42.50 \\
43.28 \\
45.54 \\
45.70 \\
47.39 \\
47.75 \\
48.56 \\
48.56\end{array}$ & $\begin{array}{l}\text { TAG-1 } \\
\text { TAG-1 } \\
\text { TAG-1 } \\
\text { TAG-1 } \\
\text { TAG-1 } \\
\text { TAG-1 } \\
\text { TAG-1 } \\
\text { TAG-1 } \\
\text { TAG-1 } \\
\text { TAG-1 } \\
\text { TAG-1 } \\
\text { TAG-1 } \\
\text { TAG-1 } \\
\text { TAG-1 } \\
\text { TAG-1 } \\
\text { TAG-1 } \\
\text { TAG-1 } \\
\text { TAG-1 } \\
\text { TAG-1 } \\
\text { TAG-1 } \\
\text { TAG-1 }\end{array}$ & $\begin{array}{l}\text { Pyrite-anhydrite breccia } \\
\text { (Sampling duplicate) } \\
\text { Pyrite-anhydrite breccia } \\
\text { Pyrite-anhydrite breccia } \\
\text { Pyrite-anhydrite breccia } \\
\text { Pyrite-silica breccia } \\
\text { Pyrite-silica breccia } \\
\text { Pyrite-silica breccia } \\
\text { Pyrite-silica breccia } \\
\text { Pyrite-silica breccia } \\
\text { Pyrite-silica breccia } \\
\text { Pyrite-silica breccia } \\
\text { Pyrite-silica breccia } \\
\text { Pyrite-silica breccia } \\
\text { Silicified wallrock breccia } \\
\text { Silicified wallrock breccia } \\
\text { Silicified wallrock breccia } \\
\text { Silicified wallrock breccia } \\
\text { Silicified wallrock breccia } \\
\text { Silicified wallrock breccia } \\
\text { (Laboratory duplicate) }\end{array}$ \\
\hline $\begin{array}{l}158-957 \mathrm{E}- \\
1 \mathrm{R}-1,23-26 \\
9 \mathrm{R}-1,16-19 \\
12 \mathrm{R}-1,16-20 \\
15 \mathrm{R}-1,27-30\end{array}$ & $\begin{array}{l}4 \\
4 \\
3 \\
6\end{array}$ & $\begin{array}{r}31.70 \\
78.16 \\
91.95 \\
106.77\end{array}$ & $\begin{array}{l}\text { TAG-1 } \\
\text { TAG-1 } \\
\text { TAG-1 } \\
\text { TAG-1 }\end{array}$ & $\begin{array}{l}\text { Pyrite-silica breccia } \\
\text { Silicified wallrock breccia } \\
\text { Silicified wallrock breccia } \\
\text { Chloritized basalt breccia }\end{array}$ \\
\hline $\begin{array}{l}158-957 \mathrm{~F}- \\
1 \mathrm{~N}-1,32-35 \\
2 \mathrm{~N}-1,11-19\end{array}$ & $\begin{array}{l}8 \\
3\end{array}$ & $\begin{array}{l}1.41 \\
5.60\end{array}$ & $\begin{array}{l}\text { TAG-1 } \\
\text { TAG-1 }\end{array}$ & $\begin{array}{l}\text { Massive pyrite breccia } \\
\text { Massive pyrite breccia }\end{array}$ \\
\hline $\begin{array}{l}158-957 \mathrm{H}- \\
1 \mathrm{~N}-1,60-73 \\
5 \mathrm{~N}-1,61-64 \\
6 \mathrm{~N}-1,14-19\end{array}$ & $\begin{array}{l}13 \\
6 \\
3\end{array}$ & $\begin{array}{r}9.30 \\
27.30 \\
31.34\end{array}$ & $\begin{array}{l}\text { TAG-2 } \\
\text { TAG-2 } \\
\text { TAG-2 }\end{array}$ & $\begin{array}{l}\text { Massive pyrite breccia } \\
\text { Pyrite-silica breccia } \\
\text { Pyrite-silica breccia }\end{array}$ \\
\hline $\begin{array}{l}\text { 158-957I- } \\
1 \mathrm{~N}-1,33-41\end{array}$ & 6 & 9.33 & TAG-4 & Massive porous pyrite+sphalerite \\
\hline $\begin{array}{l}158-957 \mathrm{~K}- \\
1 \mathrm{X}-1,24-29 \\
1 \mathrm{~N}-1,37-41 \\
3 \mathrm{X}-1,36-38\end{array}$ & $\begin{array}{l}4 \\
9 \\
7\end{array}$ & $\begin{array}{r}0.21 \\
10.37 \\
14.82\end{array}$ & $\begin{array}{l}\text { TAG-4 } \\
\text { TAG-4 } \\
\text { TAG-4 }\end{array}$ & $\begin{array}{l}\text { Porous pyrite, red and grey chert } \\
\text { Massive porous pyrite+sphalerite } \\
\text { Massive pyrite breccia }\end{array}$ \\
\hline $\begin{array}{l}\text { 158-957M- } \\
\text { 1R-1, 16-47 } \\
\text { 1R-1, 16-47 } \\
\text { 1R-1, 16-47 } \\
\text { 1R-2, 8-12 } \\
\text { 2R-1, 2-5 } \\
\text { 3R-1, 57-60 } \\
\text { 3R-1, 123-125 } \\
\text { 3R-2, } 1-4 \\
\text { 5R-1, 90-92 } \\
\text { 7R-1, 0-4 } \\
\text { 8R-1, 0-4 }\end{array}$ & $\begin{array}{c}-63 \\
420-63 \\
+420 \\
3 \\
1 \\
14 \\
28 \\
1 \\
15 \\
1 \\
1\end{array}$ & $\begin{array}{r}0.00 \\
0.00 \\
0.00 \\
0.63 \\
9.30 \\
14.87 \\
15.49 \\
15.80 \\
25.18 \\
34.30 \\
38.30\end{array}$ & $\begin{array}{l}\text { TAG-4 } \\
\text { TAG-4 } \\
\text { TAG-4 } \\
\text { TAG-4 } \\
\text { TAG-4 } \\
\text { TAG-4 } \\
\text { TAG-4 } \\
\text { TAG-4 } \\
\text { TAG-4 } \\
\text { TAG-4 } \\
\text { TAG-4 }\end{array}$ & $\begin{array}{l}\text { Porous pyrite, red and grey chert } \\
\text { Porous pyrite, red and grey chert } \\
\text { Porous pyrite, red and grey chert } \\
\text { Red chert } \\
\text { Massive porous pyrite+sphalerite } \\
\text { Massive pyrite, pyrite-silica breccias } \\
\text { Pyrite-silica breccia } \\
\text { Pyrite-silica breccia } \\
\text { Pyrite-silica breccia } \\
\text { Grey chert fragment } \\
\text { Massive granular pyrite }\end{array}$ \\
\hline $\begin{array}{l}158-957 \mathrm{O}- \\
2 \mathrm{R}-1,22-24 \\
4 \mathrm{R}-1,45-50\end{array}$ & $\begin{array}{l}5 \\
9\end{array}$ & $\begin{array}{r}8.10 \\
16.35\end{array}$ & $\begin{array}{l}\text { TAG-5 } \\
\text { TAG-5 }\end{array}$ & $\begin{array}{l}\text { Nodular, pyrite-anhydrite breccia } \\
\text { Pyrite-anhydrite breccia }\end{array}$ \\
\hline $\begin{array}{l}\text { 158-957P- } \\
\text { 1R-1, 44-46 } \\
\text { 5R-1, 9-13 } \\
\text { 5R-1, 9-13 } \\
9 \mathrm{R}-1,11-13 \\
\text { 10R-1, 1-3 } \\
\text { 12R-1, 15-100 } \\
\text { 12R-1, 15-100 } \\
\text { 12R-1, 15-100 } \\
\text { 12R-2, 25-28 } \\
\text { 12R-4, 53-57 } \\
13 \mathrm{~W}-1,50-58 \\
13 \mathrm{~W} 1+2,0-150\end{array}$ & $\begin{array}{c}8 \\
2 \\
2 \\
2 \\
1 \\
-63 \\
420-63 \\
+420 \\
6 \\
5 \\
- \\
-\end{array}$ & $\begin{array}{r}0.43 \\
21.57 \\
21.57 \\
40.19 \\
45.10 \\
54.40 \\
54.40 \\
54.40 \\
55.89 \\
57.61 \\
15.00 \\
15.00\end{array}$ & $\begin{array}{l}\text { TAG-5 } \\
\text { TAG-5 } \\
\text { TAG-5 } \\
\text { TAG-5 } \\
\text { TAG-5 } \\
\text { TAG-5 } \\
\text { TAG-5 } \\
\text { TAG-5 } \\
\text { TAG-5 } \\
\text { TAG-5 } \\
\text { TAG-5 } \\
\text { TAG-5 }\end{array}$ & $\begin{array}{l}\text { Pyrite-anhydrite breccia } \\
\text { Massive granular pyrite } \\
\text { (Laboratory duplicate) } \\
\text { Nodular, pyrite-silica breccia } \\
\text { Massive granular pyrite } \\
\text { Pyrite, silica drill cuttings } \\
\text { Pyrite, silica drill cuttings } \\
\text { Pyrite, silica drill cuttings } \\
\text { Pyrite-silica breccia } \\
\text { Massive granular pyrite } \\
\text { Pyrite, anhydrite, chert cuttings } \\
\text { (Sampling duplicate) }\end{array}$ \\
\hline $\begin{array}{l}\text { 158-957Q- } \\
\text { 1R-1, 13-18 } \\
\text { 1R-3, 70-100 } \\
\text { 1R-3, 119-121 } \\
\text { 2R-1, } 0-4\end{array}$ & $\frac{-}{1}$ & $\begin{array}{l}0.13 \\
3.75 \\
4.10 \\
9.50\end{array}$ & $\begin{array}{l}\text { TAG-3 } \\
\text { TAG-3 } \\
\text { TAG-3 } \\
\text { TAG-3 }\end{array}$ & $\begin{array}{l}\text { Fe-oxides and chert cuttings } \\
\text { Fe-oxides and chert cuttings } \\
\text { (Sampling duplicate) } \\
\text { Red chert fragment }\end{array}$ \\
\hline
\end{tabular}

Notes: * = major elements reported as oxides. ND = element not determined, $<=$ values below reported detection, $\mathrm{Fe}_{2} \mathrm{O}_{3}(\mathrm{~T})=$ total $\mathrm{Fe}$ reported as $\mathrm{Fe}_{2} \mathrm{O}_{3}, \mathrm{~S}(\mathrm{~T})=$ total sulfur, $\mathrm{LOI}=$ loss on ignition. 
Table 1 (continued).

\begin{tabular}{|c|c|c|c|c|c|c|c|c|c|c|c|c|c|}
\hline $\begin{array}{l}\text { Core, section, } \\
\text { interval }(\mathrm{cm})\end{array}$ & $\begin{array}{l}\text { Element*: } \\
\text { Method: } \\
\text { Detection } \\
\text { limit: }\end{array}$ & $\begin{array}{c}\mathrm{SiO}_{2} \\
\text { ICP-ES } \\
0.50 \%\end{array}$ & $\begin{array}{c}\mathrm{TiO}_{2} \\
\text { ICP-ES }\end{array}$ & $\begin{array}{c}\mathrm{Al}_{2} \mathrm{O}_{3} \\
\text { ICP-ES }\end{array}$ & $\begin{array}{c}\mathrm{Fe}_{2} \mathrm{O}_{3}(\mathrm{~T}) \\
\text { ICP-ES }\end{array}$ & $\begin{array}{c}\mathrm{MnO} \\
\text { ICP-ES }\end{array}$ & $\begin{array}{c}\mathrm{MgO} \\
\text { ICP-ES }\end{array}$ & $\begin{array}{c}\mathrm{CaO} \\
\text { ICP-ES }\end{array}$ & $\begin{array}{c}\mathrm{Na}_{2} \mathrm{O} \\
\text { ICP-ES } \\
0.01 \%\end{array}$ & $\begin{array}{c}\mathrm{K}_{2} \mathrm{O} \\
\text { ICP-ES } \\
0.05 \%\end{array}$ & $\begin{array}{c}\mathrm{P}_{2} \mathrm{O}_{5} \\
\text { ICP-ES }\end{array}$ & $\begin{array}{c}\mathrm{S}(\mathrm{T}) \\
\mathrm{LECO}\end{array}$ & $0.1 \%$ \\
\hline $\begin{array}{l}158-957 \mathrm{~A}- \\
3 \mathrm{X}-1,4-8\end{array}$ & & 54.00 & $<0.02$ & 0.31 & 26.4 & 0.01 & 0.26 & 0.62 & 0.62 & 0.11 & 0.32 & ND & 13.6 \\
\hline $\begin{array}{l}\text { 158-957B- } \\
1 \mathrm{R}-1,3-95 \\
1 \mathrm{R}-1,3-95 \\
1 \mathrm{R}-1,3-95 \\
1 \mathrm{R}-1,3-95 \\
4 \mathrm{R}-1,0-10\end{array}$ & & $\begin{array}{r}13.50 \\
8.40 \\
35.00 \\
34.80 \\
31.60\end{array}$ & $\begin{array}{r}0.03 \\
<0.02 \\
<0.02 \\
<0.02 \\
1.92\end{array}$ & $\begin{array}{r}0.68 \\
0.33 \\
0.28 \\
0.27 \\
16.80\end{array}$ & $\begin{array}{l}42.9 \\
44.0 \\
28.5 \\
28.4 \\
15.6\end{array}$ & $\begin{array}{l}0.01 \\
0.01 \\
0.01 \\
0.01 \\
0.06\end{array}$ & $\begin{array}{r}0.54 \\
0.31 \\
0.41 \\
0.40 \\
23.10\end{array}$ & $\begin{array}{l}0.44 \\
0.80 \\
1.66 \\
1.63 \\
0.13\end{array}$ & $\begin{array}{l}1.02 \\
0.64 \\
0.73 \\
0.42 \\
0.51\end{array}$ & $\begin{array}{l}0.09 \\
0.05 \\
0.07 \\
0.13 \\
0.06\end{array}$ & $\begin{array}{l}0.10 \\
0.06 \\
0.05 \\
0.05 \\
0.02\end{array}$ & $\begin{array}{l}33.0 \\
39.0 \\
25.5 \\
26.0 \\
\text { ND }\end{array}$ & $\begin{array}{l}20.5 \\
22.7 \\
16.4 \\
14.1 \\
11.0\end{array}$ \\
\hline $\begin{array}{l}158-957 \mathrm{C}- \\
5 \mathrm{~N}-1,15-48 \\
5 \mathrm{~N}-1,15-48 \\
7 \mathrm{~N}-1,52-56 \\
7 \mathrm{~N}-2,63-69 \\
10 \mathrm{~N}-1,21-22 \\
11 \mathrm{~N}-1,58-61 \\
11 \mathrm{~N}-3,90-94 \\
12 \mathrm{~N}-1,53-59 \\
12 \mathrm{~N}-2,68-75 \\
12 \mathrm{~N}-3,86-100 \\
13 \mathrm{~N}-1,12-20 \\
14 \mathrm{~N}-1,33-41 \\
14 \mathrm{~N}-2,76.5-88 \\
15 \mathrm{~N}-1,36-40 \\
15 \mathrm{~N}-2,12-15 \\
15 \mathrm{~N}-3,132-150 \\
15 \mathrm{~N}-4,8-10 \\
16 \mathrm{~N}-1,118-125 \\
16 \mathrm{~N}-2,14-23 \\
16 \mathrm{~N}-2,94-95 \\
16 \mathrm{~N}-2,94-95\end{array}$ & & $\begin{array}{r}0.88 \\
0.54 \\
1.21 \\
2.18 \\
12.20 \\
6.21 \\
3.60 \\
5.66 \\
1.65 \\
24.60 \\
1.06 \\
4.11 \\
15.10 \\
13.50 \\
42.10 \\
23.60 \\
19.60 \\
7.82 \\
16.00 \\
41.30 \\
41.00\end{array}$ & $\begin{array}{r}<0.02 \\
<0.02 \\
<0.02 \\
<0.02 \\
<0.02 \\
<0.02 \\
<0.02 \\
0.04 \\
<0.02 \\
0.04 \\
<0.02 \\
<0.02 \\
<0.02 \\
0.02 \\
0.08 \\
<0.02 \\
0.03 \\
<0.02 \\
0.03 \\
<0.02 \\
<0.02\end{array}$ & $\begin{array}{r}<0.20 \\
<0.20 \\
<0.20 \\
<0.20 \\
<0.20 \\
<0.20 \\
<0.20 \\
0.45 \\
<0.20 \\
0.48 \\
<0.20 \\
0.38 \\
0.26 \\
0.31 \\
0.70 \\
<0.20 \\
0.41 \\
<0.20 \\
0.45 \\
<0.20 \\
<0.20\end{array}$ & $\begin{array}{l}58.7 \\
60.5 \\
47.0 \\
35.6 \\
46.8 \\
46.1 \\
53.5 \\
51.1 \\
48.9 \\
32.9 \\
57.3 \\
52.9 \\
50.9 \\
48.4 \\
29.6 \\
40.3 \\
23.9 \\
51.6 \\
50.8 \\
37.0 \\
36.8\end{array}$ & $\begin{array}{r}0.01 \\
0.01 \\
<0.01 \\
<0.01 \\
<0.01 \\
<0.01 \\
0.01 \\
0.01 \\
<0.01 \\
<0.01 \\
0.01 \\
0.01 \\
0.01 \\
0.01 \\
<0.01 \\
<0.01 \\
<0.01 \\
0.01 \\
0.01 \\
<0.01 \\
<0.01\end{array}$ & $\begin{array}{r}0.05 \\
0.05 \\
<0.04 \\
<0.04 \\
<0.04 \\
<0.04 \\
0.09 \\
0.27 \\
0.08 \\
0.34 \\
0.15 \\
0.38 \\
0.13 \\
0.15 \\
0.12 \\
0.09 \\
0.17 \\
0.10 \\
0.15 \\
<0.04 \\
<0.04\end{array}$ & $\begin{array}{r}0.30 \\
0.31 \\
7.26 \\
14.90 \\
4.45 \\
6.96 \\
1.98 \\
3.43 \\
7.03 \\
6.39 \\
2.49 \\
3.85 \\
1.87 \\
3.00 \\
3.84 \\
4.31 \\
15.80 \\
1.89 \\
0.62 \\
0.29 \\
0.29\end{array}$ & $\begin{array}{l}0.09 \\
0.10 \\
0.06 \\
0.10 \\
0.12 \\
0.09 \\
0.08 \\
0.15 \\
0.13 \\
0.23 \\
0.07 \\
0.10 \\
0.12 \\
0.11 \\
0.16 \\
0.14 \\
0.13 \\
0.12 \\
0.16 \\
0.06 \\
0.08\end{array}$ & $\begin{array}{l}<0.05 \\
<0.05 \\
<0.05 \\
<0.05 \\
<0.05 \\
<0.05 \\
<0.05 \\
<0.05 \\
<0.05 \\
<0.05 \\
<0.05 \\
<0.05 \\
<0.05 \\
<0.05 \\
<0.05 \\
<0.05 \\
<0.05 \\
<0.05 \\
<0.05 \\
<0.05 \\
<0.05\end{array}$ & $\begin{array}{r}0.01 \\
0.02 \\
0.01 \\
0.02 \\
<0.01 \\
0.01 \\
0.02 \\
0.01 \\
0.01 \\
<0.01 \\
<0.01 \\
0.02 \\
0.02 \\
0.02 \\
0.01 \\
<0.01 \\
0.01 \\
0.01 \\
0.01 \\
<0.01 \\
0.01\end{array}$ & $\begin{array}{l}53.3 \\
53.3 \\
45.8 \\
41.2 \\
44.2 \\
45.1 \\
47.9 \\
46.5 \\
47.6 \\
33.6 \\
51.9 \\
48.4 \\
44.5 \\
44.2 \\
27.5 \\
37.7 \\
31.4 \\
45.8 \\
43.4 \\
31.8 \\
32.9\end{array}$ & $\begin{array}{l}33.4 \\
33.2 \\
25.3 \\
18.9 \\
25.8 \\
26.0 \\
28.4 \\
27.7 \\
26.9 \\
19.7 \\
30.2 \\
28.6 \\
27.6 \\
25.9 \\
16.4 \\
21.6 \\
13.2 \\
27.5 \\
26.8 \\
19.4 \\
19.2\end{array}$ \\
\hline $\begin{array}{l}158-957 \mathrm{E}- \\
1 \mathrm{R}-1,23-26 \\
9 \mathrm{R}-1,16-19 \\
12 \mathrm{R}-1,16-20 \\
15 \mathrm{R}-1,27-30\end{array}$ & & $\begin{array}{r}49.80 \\
29.60 \\
4.57 \\
32.70\end{array}$ & $\begin{array}{r}<0.02 \\
0.02 \\
0.03 \\
0.02\end{array}$ & $\begin{array}{r}<0.20 \\
0.22 \\
0.51 \\
0.26\end{array}$ & $\begin{array}{l}27.8 \\
43.1 \\
57.1 \\
41.6\end{array}$ & $\begin{array}{r}<0.01 \\
0.01 \\
<0.01 \\
<0.01\end{array}$ & $\begin{array}{l}<0.04 \\
<0.04 \\
<0.04 \\
<0.04\end{array}$ & $\begin{array}{l}2.91 \\
0.09 \\
0.14 \\
0.26\end{array}$ & $\begin{array}{l}0.09 \\
0.12 \\
0.13 \\
0.10\end{array}$ & $\begin{array}{l}<0.05 \\
<0.05 \\
<0.05 \\
<0.05\end{array}$ & $\begin{array}{l}0.01 \\
0.01 \\
0.02 \\
0.01\end{array}$ & $\begin{array}{l}25.7 \\
35.4 \\
48.9 \\
37.1\end{array}$ & $\begin{array}{l}14.7 \\
23.1 \\
29.4 \\
22.1\end{array}$ \\
\hline $\begin{array}{l}158-957 \mathrm{~F}- \\
1 \mathrm{~N}-1,32-35 \\
2 \mathrm{~N}-1,11-19\end{array}$ & & $\begin{array}{l}<0.50 \\
<0.50\end{array}$ & $\begin{array}{l}<0.02 \\
<0.02\end{array}$ & $\begin{array}{r}<0.20 \\
0.20\end{array}$ & $\begin{array}{l}43.0 \\
52.5\end{array}$ & $\begin{array}{r}<0.01 \\
0.01\end{array}$ & $\begin{array}{r}0.09 \\
<0.04\end{array}$ & $\begin{array}{l}7.98 \\
4.45\end{array}$ & $\begin{array}{l}0.11 \\
0.08\end{array}$ & $\begin{array}{l}<0.05 \\
<0.05\end{array}$ & $\begin{array}{l}0.01 \\
0.01\end{array}$ & $\begin{array}{l}43.8 \\
48.5\end{array}$ & $\begin{array}{l}25.3 \\
27.3\end{array}$ \\
\hline $\begin{array}{l}158-957 \mathrm{H}- \\
1 \mathrm{~N}-1,60-73 \\
5 \mathrm{~N}-1,61-64 \\
6 \mathrm{~N}-1,14-19\end{array}$ & & $\begin{array}{l}<0.50 \\
19.40 \\
33.80\end{array}$ & $\begin{array}{r}<0.02 \\
0.03 \\
<0.02\end{array}$ & $\begin{array}{r}<0.20 \\
0.46 \\
<0.20\end{array}$ & $\begin{array}{l}42.5 \\
48.0 \\
39.1\end{array}$ & $\begin{array}{r}<0.01 \\
0.01 \\
0.01\end{array}$ & $\begin{array}{r}<0.04 \\
0.06 \\
0.05\end{array}$ & $\begin{array}{l}9.04 \\
0.79 \\
0.09\end{array}$ & $\begin{array}{l}0.11 \\
0.16 \\
0.08\end{array}$ & $\begin{array}{l}<0.05 \\
<0.05 \\
<0.05\end{array}$ & $\begin{array}{r}0.01 \\
0.01 \\
<0.01\end{array}$ & $\begin{array}{l}44.4 \\
42.3 \\
33.5\end{array}$ & $\begin{array}{l}22.0 \\
25.6 \\
19.5\end{array}$ \\
\hline $\begin{array}{l}158-957 \mathrm{I}- \\
1 \mathrm{~N}-1,33-41\end{array}$ & & $<0.50$ & $<0.02$ & $<0.20$ & 59.0 & 0.01 & $<0.04$ & 0.08 & 0.08 & $<0.05$ & 0.02 & 50.7 & 33.5 \\
\hline $\begin{array}{l}158-957 \mathrm{~K}- \\
1 \mathrm{X}-1,24-29 \\
1 \mathrm{~N}-1,37-41 \\
3 \mathrm{X}-1,36-38\end{array}$ & & $\begin{array}{r}6.28 \\
<0.50 \\
0.61\end{array}$ & $\begin{array}{l}<0.02 \\
<0.02 \\
<0.02\end{array}$ & $\begin{array}{l}<0.20 \\
<0.20 \\
<0.20\end{array}$ & $\begin{array}{l}54.1 \\
59.3 \\
56.4\end{array}$ & $\begin{array}{l}0.01 \\
0.01 \\
0.01\end{array}$ & $\begin{array}{l}<0.04 \\
<0.04 \\
<0.04\end{array}$ & $\begin{array}{l}0.09 \\
0.10 \\
0.07\end{array}$ & $\begin{array}{l}0.10 \\
0.04 \\
0.06\end{array}$ & $\begin{array}{l}<0.05 \\
<0.05 \\
<0.05\end{array}$ & $\begin{array}{l}0.03 \\
0.02 \\
0.01\end{array}$ & $\begin{array}{l}46.4 \\
52.4 \\
51.9\end{array}$ & $\begin{array}{l}31.9 \\
33.1 \\
33.6\end{array}$ \\
\hline $\begin{array}{c}\text { 158-957M- } \\
\text { 1R-1, 16-47 } \\
\text { 1R-1, 16-47 } \\
\text { 1R-1, 16-47 } \\
\text { 1R-2, 8-12 } \\
\text { 2R-1, 2-5 } \\
\text { 3R-1, 57-60 } \\
\text { 3R-1, 123-125 } \\
\text { 3R-2, 1-4 } \\
\text { 5R-1, 90-92 } \\
\text { 7R-1, 0-4 } \\
\text { 8R-1, 0-4 }\end{array}$ & & $\begin{array}{r}6.04 \\
6.63 \\
6.87 \\
77.40 \\
0.55 \\
3.09 \\
22.00 \\
32.80 \\
45.60 \\
76.60 \\
14.00\end{array}$ & $\begin{array}{r}<0.02 \\
<0.02 \\
<0.02 \\
0.03 \\
<0.02 \\
<0.02 \\
<0.02 \\
<0.02 \\
0.04 \\
0.03 \\
<0.02\end{array}$ & $\begin{array}{r}<0.20 \\
<0.20 \\
<0.20 \\
<0.20 \\
<0.20 \\
<0.20 \\
<0.20 \\
<0.20 \\
0.48 \\
0.42 \\
<0.20\end{array}$ & $\begin{array}{l}68.8 \\
67.6 \\
68.9 \\
16.1 \\
59.1 \\
56.3 \\
45.3 \\
39.1 \\
32.3 \\
15.2 \\
51.4\end{array}$ & $\begin{array}{r}0.04 \\
0.04 \\
0.05 \\
<0.01 \\
<0.01 \\
0.01 \\
0.01 \\
<0.01 \\
<0.01 \\
<0.01 \\
0.01\end{array}$ & $\begin{array}{r}0.56 \\
0.57 \\
0.60 \\
0.12 \\
0.24 \\
<0.04 \\
<0.04 \\
<0.04 \\
<0.04 \\
0.10 \\
<0.04\end{array}$ & $\begin{array}{l}0.35 \\
0.36 \\
0.34 \\
0.07 \\
0.06 \\
0.08 \\
0.07 \\
0.07 \\
0.07 \\
0.06 \\
0.08\end{array}$ & $\begin{array}{l}0.70 \\
0.56 \\
0.76 \\
0.06 \\
0.05 \\
0.08 \\
0.04 \\
0.07 \\
0.12 \\
0.03 \\
0.06\end{array}$ & $\begin{array}{r}0.19 \\
0.17 \\
0.18 \\
<0.05 \\
<0.05 \\
<0.05 \\
<0.05 \\
<0.05 \\
<0.05 \\
<0.05 \\
<0.05\end{array}$ & $\begin{array}{r}0.60 \\
0.62 \\
0.61 \\
<0.01 \\
0.02 \\
0.01 \\
0.02 \\
<0.01 \\
<0.01 \\
<0.01 \\
0.01\end{array}$ & $\begin{array}{l}5.01 \\
1.33 \\
0.64 \\
\text { ND } \\
\text { ND } \\
46.0 \\
39.8 \\
34.0 \\
28.0 \\
\text { ND } \\
44.9\end{array}$ & $\begin{array}{c}11.8 \\
12.1 \\
10.2 \\
5.9 \\
30.8 \\
31.0 \\
24.8 \\
20.1 \\
16.8 \\
7.8 \\
27.2\end{array}$ \\
\hline $\begin{array}{l}\text { 158-957O- } \\
\text { 2R-1, 22-24 } \\
4 \mathrm{R}-1,45-50\end{array}$ & & $\begin{array}{l}<0.50 \\
<0.50\end{array}$ & $\begin{array}{l}<0.02 \\
<0.02\end{array}$ & $\begin{array}{r}<0.20 \\
0.24\end{array}$ & $\begin{array}{l}42.7 \\
54.2\end{array}$ & $\begin{array}{l}<0.01 \\
<0.01\end{array}$ & $\begin{array}{l}<0.04 \\
<0.04\end{array}$ & $\begin{array}{r}10.80 \\
3.33\end{array}$ & $\begin{array}{l}0.10 \\
0.06\end{array}$ & $\begin{array}{l}<0.05 \\
<0.05\end{array}$ & $\begin{array}{r}0.01 \\
<0.01\end{array}$ & $\begin{array}{l}44.6 \\
50.6\end{array}$ & $\begin{array}{l}22.1 \\
28.7\end{array}$ \\
\hline $\begin{array}{l}\text { 158-957P- } \\
1 \mathrm{R}-1,44-46 \\
5 \mathrm{R}-1,9-13 \\
5 \mathrm{R}-1,9-13 \\
9 \mathrm{R}-1,11-13 \\
10 \mathrm{R}-1,1-3 \\
12 \mathrm{R}-1,15-100 \\
12 \mathrm{R}-1,15-100 \\
\text { 12R-1, 15-100 } \\
\text { 12R-2, 25-28 } \\
\text { 12R-4, 53-57 } \\
\text { 13W-1, 50-58 } \\
13 \mathrm{~W} 1+2,0-150\end{array}$ & & $\begin{array}{r}<0.50 \\
<0.50 \\
<0.50 \\
16.40 \\
<0.50 \\
3.12 \\
4.03 \\
11.30 \\
48.20 \\
5.26 \\
2.88 \\
3.82\end{array}$ & $\begin{array}{c}<0.02 \\
<0.02 \\
<0.02 \\
<0.02 \\
<0.02 \\
<0.02 \\
<0.02 \\
0.03 \\
0.13 \\
<0.0 \\
<0.02 \\
<0.02\end{array}$ & $\begin{array}{r}<0.20 \\
<0.20 \\
<0.20 \\
0.21 \\
0.20 \\
<0.20 \\
<0.20 \\
0.33 \\
1.13 \\
20.44 \\
<0.20 \\
0.23\end{array}$ & $\begin{array}{l}32.9 \\
54.4 \\
54.7 \\
51.7 \\
58.7 \\
26.5 \\
30.7 \\
43.7 \\
31.6 \\
56.6 \\
55.6 \\
52.4\end{array}$ & $\begin{array}{r}<0.01 \\
<0.01 \\
<0.01 \\
<0.01 \\
0.01 \\
0.01 \\
0.01 \\
0.01 \\
<0.01 \\
0.01 \\
0.01 \\
<0.01\end{array}$ & $\begin{array}{r}<0.04 \\
<0.04 \\
<0.04 \\
0.08 \\
0.06 \\
0.10 \\
0.09 \\
0.10 \\
0.05 \\
0.09 \\
0.07 \\
0.27\end{array}$ & $\begin{array}{r}16.10 \\
4.59 \\
4.65 \\
0.07 \\
0.33 \\
0.98 \\
2.67 \\
2.71 \\
0.08 \\
0.11 \\
0.30 \\
0.45\end{array}$ & $\begin{array}{l}0.09 \\
0.08 \\
0.07 \\
0.06 \\
0.08 \\
0.40 \\
0.33 \\
0.16 \\
0.18 \\
0.09 \\
0.21 \\
0.27\end{array}$ & $\begin{array}{r}<0.05 \\
<0.05 \\
<0.05 \\
<0.05 \\
<0.05 \\
<0.05 \\
<0.05 \\
0.05 \\
0.05 \\
<0.05 \\
0.06 \\
<0.05\end{array}$ & $\begin{array}{r}0.01 \\
0.01 \\
0.01 \\
0.01 \\
0.02 \\
0.02 \\
0.01 \\
0.01 \\
<0.01 \\
0.02 \\
0.01 \\
0.03\end{array}$ & $\begin{array}{l}41.4 \\
51.7 \\
52.4 \\
44.9 \\
52.8 \\
44.2 \\
44.9 \\
41.3 \\
27.5 \\
49.6 \\
49.4 \\
\text { ND }\end{array}$ & $\begin{array}{l}17.7 \\
30.3 \\
30.1 \\
26.7 \\
30.9 \\
23.9 \\
24.1 \\
22.3 \\
16.7 \\
29.9 \\
28.2 \\
28.7\end{array}$ \\
\hline $\begin{array}{l}\text { 158-957Q- } \\
\text { 1R-1, 13-18 } \\
\text { 1R-3, 70-100 } \\
\text { 1R-3, 119-121 } \\
\text { 2R-1, 0-4 }\end{array}$ & & $\begin{array}{l}59.90 \\
16.40 \\
80.10 \\
92.70\end{array}$ & $\begin{array}{r}0.05 \\
<0.02 \\
<0.02 \\
<0.02\end{array}$ & $\begin{array}{l}<0.20 \\
<0.20 \\
<0.20 \\
<0.20\end{array}$ & $\begin{array}{l}20.8 \\
46.5 \\
13.5 \\
5.49\end{array}$ & $\begin{array}{r}0.13 \\
0.01 \\
<0.01 \\
<0.01\end{array}$ & $\begin{array}{l}0.29 \\
0.06 \\
0.20 \\
0.15\end{array}$ & $\begin{array}{l}0.20 \\
0.19 \\
0.15 \\
0.06\end{array}$ & $\begin{array}{l}0.38 \\
0.19 \\
0.54 \\
0.06\end{array}$ & $\begin{array}{r}0.07 \\
0.05 \\
0.07 \\
<0.05\end{array}$ & $\begin{array}{r}0.08 \\
0.03 \\
<0.01 \\
<0.01\end{array}$ & $\begin{array}{l}\text { ND } \\
41.2 \\
\text { ND } \\
\text { ND }\end{array}$ & $\begin{array}{c}10.0 \\
23.0 \\
5.9 \\
2.8\end{array}$ \\
\hline
\end{tabular}


Table 2. Trace element composition of samples from Site 957, TAG hydrothermal field, Mid-Atlantic Ridge.

\begin{tabular}{|c|c|c|c|c|c|c|c|c|c|c|c|c|c|}
\hline $\begin{array}{l}\text { Core, section, } \\
\text { interval }(\mathrm{cm})\end{array}$ & Piece & $\begin{array}{l}\text { Depth } \\
\text { (mbsf) }\end{array}$ & $\begin{array}{l}\text { Element: } \\
\text { Method: } \\
\text { Detection } \\
\text { limit: }\end{array}$ & $\begin{array}{c}\mathrm{Cu} \\
\text { ICP-ES } \\
10 \mathrm{ppm}\end{array}$ & $\begin{array}{c}\mathrm{Zn} \\
\text { ICP-ES } \\
5 \mathrm{ppm}\end{array}$ & $\begin{array}{c}\mathrm{Ni} \\
\text { ICP-ES } \\
10 \mathrm{ppm}\end{array}$ & $\begin{array}{c}\text { Co } \\
\text { ICP-ES } \\
5 \mathrm{ppm}\end{array}$ & $\begin{array}{c}\mathrm{Ba} \\
\text { ICP-ES } \\
10 \mathrm{ppm}\end{array}$ & $\begin{array}{c}\mathrm{Sr} \\
\text { ICP-ES } \\
20 \mathrm{ppm}\end{array}$ & $\begin{array}{c}\mathrm{V} \\
\text { ICP-ES } \\
5 \mathrm{ppm}\end{array}$ & $\begin{array}{c}\mathrm{Be} \\
\text { ICP-ES } \\
0.5 \mathrm{ppm}\end{array}$ & $\begin{array}{c}\mathrm{Sc} \\
\text { ICP-ES } \\
0.5 \mathrm{ppm}\end{array}$ & $\begin{array}{c}\mathrm{Cr}^{*} \\
\text { ICP-ES } \\
10 \mathrm{ppm}\end{array}$ \\
\hline $\begin{array}{r}\text { 158-957A- } \\
3 \mathrm{X}-1,4-8\end{array}$ & 1 & 10.00 & & 3300 & 19000 & $<10$ & 10 & 40 & $<20$ & 240 & 0.5 & 0.8 & 21 \\
\hline $\begin{array}{c}\text { 158-957B- } \\
1 \mathrm{R}-1,3-95 \\
\text { 1R-1, 3-95 } \\
\text { 1R-1, 3-95 } \\
\text { 1R-1, 3-95 } \\
\text { 4R-1, 0-10 }\end{array}$ & $\begin{array}{c}-63 \\
63-420 \\
+420 \\
+420 \\
1\end{array}$ & $\begin{array}{r}0.00 \\
0.00 \\
0.00 \\
0.00 \\
19.90\end{array}$ & & $\begin{array}{r}120000 \\
118000 \\
73000 \\
73000 \\
34\end{array}$ & $\begin{array}{r}15000 \\
30000 \\
26000 \\
26000 \\
210\end{array}$ & $\begin{array}{l}<10 \\
<10 \\
<10 \\
<10 \\
150\end{array}$ & $\begin{array}{l}68 \\
51 \\
40 \\
39 \\
45\end{array}$ & $\begin{array}{r}80 \\
90 \\
60 \\
60 \\
<30\end{array}$ & $\begin{array}{r}24 \\
38 \\
73 \\
72 \\
<20\end{array}$ & $\begin{array}{l}400 \\
200 \\
150 \\
150 \\
220\end{array}$ & $\begin{array}{r}<0.5 \\
<0.5 \\
<0.5 \\
<0.5 \\
0.9\end{array}$ & $\begin{array}{l}4.5 \\
3.8 \\
2.4 \\
2.5 \\
37\end{array}$ & $\begin{array}{r}63 \\
51 \\
43 \\
31 \\
330\end{array}$ \\
\hline $\begin{array}{c}158-957 \mathrm{C}- \\
5 \mathrm{~N}-1,15-48 \\
5 \mathrm{~N}-1,15-48 \\
7 \mathrm{~N}-1,52-56 \\
7 \mathrm{~N}-2,63-69 \\
10 \mathrm{~N}-1,21-22 \\
11 \mathrm{~N}-1,58-61 \\
11 \mathrm{~N}-3,90-94 \\
12 \mathrm{~N}-1,53-59 \\
12 \mathrm{~N}-2,68-75 \\
12 \mathrm{~N}-3,86-100 \\
13 \mathrm{~N}-1,12-20 \\
14 \mathrm{~N}-1,33-41 \\
14 \mathrm{~N}-2,76.5-88 \\
15 \mathrm{~N}-1,36-40 \\
15 \mathrm{~N}-2,12-15 \\
15 \mathrm{~N}-3,132-150 \\
15 \mathrm{~N}-4,8-10 \\
16 \mathrm{~N}-1,118-125 \\
16 \mathrm{~N}-2,14-23 \\
16 \mathrm{~N}-2,94-95 \\
16 \mathrm{~N}-2,94-95\end{array}$ & $\begin{array}{c}3,5,6 \\
3,5,6 \\
6 \mathrm{~F} \\
1 \mathrm{E} \\
4 \\
3 \mathrm{D} \\
8 \mathrm{C} \\
6 \mathrm{~A} \\
9 \\
9 \\
4 \\
5 \\
6 \\
5 \\
1 \mathrm{~B} \\
11 \\
1 \\
15 \\
2 \\
9 \mathrm{~B} \\
9 \mathrm{~B}\end{array}$ & $\begin{array}{l}15.25 \\
15.25 \\
20.04 \\
21.45 \\
28.86 \\
31.18 \\
34.50 \\
35.45 \\
36.16 \\
36.94 \\
37.31 \\
40.53 \\
41.67 \\
42.50 \\
43.28 \\
45.54 \\
45.70 \\
47.39 \\
47.75 \\
48.56 \\
48.56\end{array}$ & & $\begin{array}{r}7800 \\
7900 \\
45000 \\
34000 \\
19000 \\
23000 \\
54000 \\
31000 \\
18000 \\
24000 \\
19000 \\
17000 \\
2100 \\
5700 \\
96 \\
12000 \\
14000 \\
33000 \\
14000 \\
1100 \\
1100\end{array}$ & $\begin{array}{r}490 \\
490 \\
270 \\
300 \\
260 \\
180 \\
640 \\
650 \\
1600 \\
73 \\
730 \\
320 \\
410 \\
500 \\
31 \\
44 \\
40 \\
350 \\
130 \\
41 \\
40\end{array}$ & $\begin{array}{l}<10 \\
<10 \\
<10 \\
<10 \\
<10 \\
<10 \\
<10 \\
<10 \\
<10 \\
<10 \\
<10 \\
<10 \\
<10 \\
<10 \\
<10 \\
<10 \\
<10 \\
<10 \\
<10 \\
<10 \\
<10\end{array}$ & $\begin{array}{l}420 \\
420 \\
270 \\
130 \\
160 \\
130 \\
170 \\
220 \\
170 \\
260 \\
160 \\
160 \\
250 \\
230 \\
380 \\
300 \\
150 \\
150 \\
370 \\
240 \\
230\end{array}$ & $\begin{array}{r}130 \\
120 \\
90 \\
70 \\
80 \\
80 \\
100 \\
100 \\
90 \\
70 \\
100 \\
100 \\
90 \\
90 \\
60 \\
80 \\
60 \\
90 \\
100 \\
70 \\
70\end{array}$ & $\begin{array}{r}<20 \\
<20 \\
350 \\
570 \\
180 \\
250 \\
83 \\
160 \\
290 \\
290 \\
100 \\
140 \\
69 \\
110 \\
160 \\
160 \\
620 \\
64 \\
20 \\
<20 \\
<20\end{array}$ & $\begin{array}{r}11 \\
12 \\
5 \\
6 \\
<5 \\
6 \\
65 \\
11 \\
<5 \\
12 \\
6 \\
9 \\
<5 \\
6 \\
6 \\
13 \\
<5 \\
6 \\
<5 \\
8 \\
8 \\
<5 \\
<5\end{array}$ & $\begin{array}{l}<0.5 \\
<0.5 \\
<0.5 \\
<0.5 \\
<0.5 \\
<0.5 \\
<0.5 \\
<0.5 \\
<0.5 \\
<0.5 \\
<0.5 \\
<0.5 \\
<0.5 \\
<0.5 \\
<0.5 \\
<0.5 \\
<0.5 \\
<0.5 \\
<0.5 \\
<0.5 \\
<0.5\end{array}$ & $\begin{array}{l}4.4 \\
4.4 \\
3.5 \\
2.9 \\
3.3 \\
3.4 \\
3.9 \\
3.7 \\
3.3 \\
2.5 \\
4.3 \\
4.0 \\
3.8 \\
3.8 \\
2.4 \\
3.2 \\
2.4 \\
4.2 \\
4.1 \\
2.9 \\
2.8\end{array}$ & $\begin{array}{l}29 \\
26 \\
27 \\
18 \\
22 \\
21 \\
19 \\
30 \\
22 \\
32 \\
22 \\
22 \\
28 \\
23 \\
48 \\
24 \\
17 \\
25 \\
32 \\
27 \\
25\end{array}$ \\
\hline $\begin{array}{c}\text { 158-957E- } \\
1 \mathrm{R}-1,23-26 \\
9 \mathrm{R}-1,16-19 \\
12 \mathrm{R}-1,16-20 \\
15 \mathrm{R}-1,27-30\end{array}$ & $\begin{array}{l}4 \\
4 \\
3 \\
6\end{array}$ & $\begin{array}{r}31.70 \\
78.16 \\
91.95 \\
106.77\end{array}$ & & $\begin{array}{r}580 \\
6100 \\
38000 \\
300\end{array}$ & $\begin{array}{r}45 \\
120 \\
150 \\
120\end{array}$ & $\begin{array}{l}<10 \\
<10 \\
<10 \\
<10\end{array}$ & $\begin{array}{l}240 \\
350 \\
310 \\
380\end{array}$ & $\begin{array}{r}60 \\
80 \\
110 \\
80\end{array}$ & $\begin{array}{l}130 \\
<20 \\
<20 \\
<20\end{array}$ & $\begin{array}{r}<5 \\
5 \\
11 \\
<5\end{array}$ & $\begin{array}{l}<0.5 \\
<0.5 \\
<0.5 \\
<0.5\end{array}$ & $\begin{array}{l}2.4 \\
3.4 \\
4.7 \\
3.4\end{array}$ & $\begin{array}{l}30 \\
28 \\
28 \\
20\end{array}$ \\
\hline $\begin{array}{l}158-957 \mathrm{~F}- \\
1 \mathrm{~N}-1,32-35 \\
2 \mathrm{~N}-1,11-19\end{array}$ & $\begin{array}{l}8 \\
3\end{array}$ & $\begin{array}{l}1.41 \\
5.60\end{array}$ & & $\begin{array}{l}49000 \\
40000\end{array}$ & $\begin{array}{l}290 \\
300\end{array}$ & $\begin{array}{l}<10 \\
<10\end{array}$ & $\begin{array}{l}350 \\
210\end{array}$ & $\begin{array}{r}80 \\
100\end{array}$ & $\begin{array}{l}450 \\
190\end{array}$ & $\begin{array}{l}<5 \\
13\end{array}$ & $\begin{array}{l}<0.5 \\
<0.5\end{array}$ & $\begin{array}{l}4.1 \\
4.3\end{array}$ & $\begin{array}{r}<10 \\
18\end{array}$ \\
\hline $\begin{array}{l}158-957 \mathrm{H}- \\
1 \mathrm{~N}-1,60-73 \\
5 \mathrm{~N}-1,61-64 \\
6 \mathrm{~N}-1,14-19\end{array}$ & $\begin{array}{c}13 \\
6 \\
3\end{array}$ & $\begin{array}{r}9.30 \\
27.30 \\
31.34\end{array}$ & & $\begin{array}{r}63000 \\
4500 \\
38000\end{array}$ & $\begin{array}{l}150 \\
200 \\
150\end{array}$ & $\begin{array}{l}<10 \\
<10 \\
<10\end{array}$ & $\begin{array}{l}170 \\
350 \\
220\end{array}$ & $\begin{array}{l}80 \\
90 \\
80\end{array}$ & $\begin{array}{r}460 \\
39 \\
<20\end{array}$ & $\begin{array}{r}11 \\
10 \\
5\end{array}$ & $\begin{array}{l}<0.5 \\
<0.5 \\
<0.5\end{array}$ & $\begin{array}{l}3.9 \\
3.7 \\
3.3\end{array}$ & $\begin{array}{l}12 \\
35 \\
28\end{array}$ \\
\hline $\begin{array}{l}158-957 \mathrm{I}- \\
1 \mathrm{~N}-1,33-41\end{array}$ & 6 & 9.33 & & 1300 & 34000 & $<10$ & 30 & 100 & $<20$ & $<5$ & $<0.5$ & 4.4 & 22 \\
\hline $\begin{array}{l}158-957 \mathrm{~K}- \\
1 \mathrm{X}-1,24-29 \\
1 \mathrm{~N}-1,37-41 \\
3 \mathrm{X}-1,36-38\end{array}$ & $\begin{array}{l}4 \\
9 \\
7\end{array}$ & $\begin{array}{r}0.21 \\
10.37 \\
14.82\end{array}$ & & $\begin{array}{r}390 \\
10000 \\
710\end{array}$ & $\begin{array}{r}29000 \\
7200 \\
37000\end{array}$ & $\begin{array}{r}<10 \\
<10 \\
19\end{array}$ & $\begin{array}{l}15 \\
51 \\
49\end{array}$ & $\begin{array}{l}100 \\
130 \\
110\end{array}$ & $\begin{array}{l}<20 \\
<20 \\
<20\end{array}$ & $\begin{array}{r}<5 \\
8 \\
<5\end{array}$ & $\begin{array}{l}<0.5 \\
<0.5 \\
<0.5\end{array}$ & $\begin{array}{l}4.2 \\
4.7 \\
4.6\end{array}$ & $\begin{array}{l}19 \\
24 \\
27\end{array}$ \\
\hline $\begin{array}{l}\text { 158-957M- } \\
\text { 1R-1, 16-47 } \\
\text { 1R-1, 16-47 } \\
\text { 1R-1, 16-47 } \\
\text { 1R-2, 8-12 } \\
\text { 2R-1, 2-5 } \\
\text { 3R-1, 57-60 } \\
\text { 3R-1, 123-125 } \\
\text { 3R-2, 1-4 } \\
\text { 5R-1, 90-92 } \\
\text { 7R-1, }-4 \\
\text { 8R-1, }-4\end{array}$ & $\begin{array}{c}-63 \\
420-63 \\
+420 \\
3 \\
1 \\
14 \\
28 \\
1 \\
15 \\
1 \\
1\end{array}$ & $\begin{array}{r}0.00 \\
0.00 \\
0.00 \\
0.63 \\
9.30 \\
14.87 \\
15.49 \\
15.80 \\
25.18 \\
34.30 \\
38.30\end{array}$ & & $\begin{array}{r}2400 \\
1700 \\
860 \\
74 \\
44000 \\
3500 \\
15000 \\
36000 \\
13000 \\
1900 \\
18000\end{array}$ & $\begin{array}{r}2100 \\
590 \\
500 \\
190 \\
450 \\
41000 \\
7600 \\
330 \\
410 \\
380 \\
6300\end{array}$ & $\begin{array}{l}<10 \\
<10 \\
<10 \\
<10 \\
<10 \\
<10 \\
<10 \\
<10 \\
<10 \\
14 \\
<10\end{array}$ & $\begin{array}{r}14 \\
12 \\
8 \\
<5 \\
120 \\
74 \\
200 \\
370 \\
250 \\
68 \\
220\end{array}$ & $\begin{array}{r}560 \\
160 \\
380 \\
<30 \\
<30 \\
170 \\
110 \\
80 \\
70 \\
<30 \\
150\end{array}$ & $\begin{array}{r}58 \\
48 \\
52 \\
<20 \\
<20 \\
<20 \\
<20 \\
<20 \\
<20 \\
<20 \\
<20\end{array}$ & $\begin{array}{r}140 \\
160 \\
160 \\
7 \\
9 \\
7 \\
12 \\
<5 \\
26 \\
<5 \\
16\end{array}$ & $\begin{array}{r}0.6 \\
0.8 \\
0.7 \\
0.9 \\
<0.5 \\
<0.5 \\
<0.5 \\
<0.5 \\
<0.5 \\
0.5 \\
<0.5\end{array}$ & $\begin{array}{r}1.1 \\
0.9 \\
0.9 \\
<0.5 \\
0.5 \\
4.8 \\
3.8 \\
3.0 \\
2.5 \\
<0.5 \\
3.9\end{array}$ & $\begin{array}{r}14 \\
<10 \\
<10 \\
<10 \\
<10 \\
24 \\
28 \\
19 \\
36 \\
<10 \\
26\end{array}$ \\
\hline $\begin{array}{l}158-957 \mathrm{O}- \\
2 \mathrm{R}-1,22-24 \\
4 \mathrm{R}-1,45-50\end{array}$ & $\begin{array}{l}5 \\
9\end{array}$ & $\begin{array}{r}8.10 \\
16.35\end{array}$ & & $\begin{array}{l}34000 \\
28000\end{array}$ & $\begin{array}{l}330 \\
360\end{array}$ & $\begin{array}{l}<10 \\
<10\end{array}$ & $\begin{array}{l}200 \\
240\end{array}$ & $\begin{array}{r}80 \\
110\end{array}$ & $\begin{array}{l}680 \\
140\end{array}$ & $\begin{array}{l}<5 \\
27\end{array}$ & $\begin{array}{l}<0.5 \\
<0.5\end{array}$ & $\begin{array}{l}3.7 \\
4.6\end{array}$ & $\begin{array}{l}13 \\
18\end{array}$ \\
\hline $\begin{array}{l}\text { 158-957P- } \\
\text { 1R-1, 44-46 } \\
\text { 5R-1, 9-13 } \\
\text { 5R-1, 9-13 } \\
\text { 9R-1,11-13 } \\
\text { 10R-1, } 1-3 \\
12 \mathrm{R}-1,15-100 \\
12 \mathrm{R}-1,15-100 \\
12 \mathrm{R}-1,15-100 \\
12 \mathrm{R}-2,25-28 \\
12 \mathrm{R}-4,53-57 \\
13 \mathrm{~W}-1,50-58 \\
13 \mathrm{~W} 1+2,0-150\end{array}$ & $\begin{array}{c}8 \\
2 \\
2 \\
2 \\
1 \\
-63 \\
420-63 \\
+420 \\
6 \\
5 \\
- \\
-\end{array}$ & $\begin{array}{r}0.43 \\
21.57 \\
21.57 \\
40.19 \\
45.10 \\
54.40 \\
54.40 \\
54.40 \\
55.89 \\
57.61 \\
15.00 \\
15.00\end{array}$ & & $\begin{array}{r}42000 \\
1700 \\
1600 \\
15000 \\
33000 \\
122000 \\
92000 \\
76000 \\
360 \\
25000 \\
64000 \\
67000\end{array}$ & $\begin{array}{r}96 \\
720 \\
700 \\
280 \\
260 \\
4500 \\
3100 \\
2500 \\
85 \\
160 \\
1500 \\
1800\end{array}$ & $\begin{array}{l}<10 \\
<10 \\
<10 \\
<10 \\
<10 \\
<10 \\
<10 \\
<10 \\
<10 \\
<10 \\
<10 \\
<10\end{array}$ & $\begin{array}{l}190 \\
310 \\
300 \\
310 \\
380 \\
200 \\
230 \\
240 \\
500 \\
510 \\
350 \\
270\end{array}$ & $\begin{array}{r}70 \\
100 \\
100 \\
100 \\
120 \\
270 \\
140 \\
100 \\
60 \\
100 \\
110 \\
<30\end{array}$ & $\begin{array}{r}830 \\
170 \\
170 \\
<20 \\
24 \\
50 \\
140 \\
150 \\
<20 \\
<20 \\
<20 \\
<20\end{array}$ & $\begin{array}{r}<5 \\
<5 \\
<5 \\
<5 \\
<5 \\
20 \\
13 \\
19 \\
21 \\
35 \\
8 \\
15\end{array}$ & $\begin{array}{l}<0.5 \\
<0.5 \\
<0.5 \\
<0.5 \\
<0.5 \\
<0.5 \\
<0.5 \\
<0.5 \\
<0.5 \\
<0.5 \\
<0.5 \\
<0.5\end{array}$ & $\begin{array}{l}3.3 \\
4.1 \\
3.8 \\
3.7 \\
4.9 \\
4.1 \\
3.9 \\
3.6 \\
2.4 \\
4.2 \\
4.2 \\
<0.5\end{array}$ & $\begin{array}{l}11 \\
15 \\
14 \\
18 \\
16 \\
50 \\
32 \\
26 \\
31 \\
21 \\
18 \\
15\end{array}$ \\
\hline $\begin{array}{l}\text { 158-957Q- } \\
\text { 1R-1, 13-18 } \\
\text { 1R-3, 70-100 } \\
\text { 1R-3, 119-121 } \\
\text { 2R-1, 0-4 }\end{array}$ & $\frac{\bar{z}}{\overline{1}}$ & $\begin{array}{l}0.13 \\
3.75 \\
4.10 \\
9.50\end{array}$ & & $\begin{array}{r}32000 \\
63000 \\
5400 \\
130\end{array}$ & $\begin{array}{r}6000 \\
3600 \\
2800 \\
820\end{array}$ & $\begin{array}{l}<10 \\
<10 \\
<10 \\
35\end{array}$ & $\begin{array}{r}19 \\
120 \\
8 \\
<5\end{array}$ & $\begin{array}{r}<30 \\
90 \\
<30 \\
<30\end{array}$ & $\begin{array}{l}24 \\
<20 \\
<20 \\
<20\end{array}$ & $\begin{array}{r}54 \\
12 \\
17 \\
6\end{array}$ & $\begin{array}{r}0.8 \\
<0.5 \\
1.4 \\
1.5\end{array}$ & $\begin{array}{l}<0.5 \\
3.6 \\
<0.5 \\
<0.5\end{array}$ & $\begin{array}{r}11 \\
29 \\
<10 \\
14\end{array}$ \\
\hline
\end{tabular}

Notes: * = high values for $\mathrm{Cr}$ indicate contamination during grinding in steel mill (see text for discussion). $\mathrm{ND}=$ element not determined; $<=$ values below reported detection. 
Table 3. Trace element composition of samples from Site 957.

\begin{tabular}{|c|c|c|c|c|c|c|c|c|c|}
\hline & & & $\begin{array}{l}\text { Element: } \\
\text { Method: }\end{array}$ & $\begin{array}{c}\mathrm{Pb} \\
\text { ICP-MS }\end{array}$ & $\begin{array}{c}\mathrm{Cd} \\
\text { ICP-MS }\end{array}$ & $\begin{array}{c}\mathrm{Ag} \\
\text { ICP-MS }\end{array}$ & $\begin{array}{c}\text { In } \\
\text { ICP-MS }\end{array}$ & $\begin{array}{c}\text { Mo } \\
\text { ICP-MS }\end{array}$ & $\begin{array}{c}\text { Tl } \\
\text { ICP-MS }\end{array}$ \\
\hline $\begin{array}{l}\text { Core, section, } \\
\text { interval }(\mathrm{cm})\end{array}$ & Piece & $\begin{array}{l}\text { Depth } \\
\text { (mbsf) }\end{array}$ & $\begin{array}{l}\text { Detection } \\
\text { limit: }\end{array}$ & $0.5 \mathrm{ppm}$ & $0.5 \mathrm{ppm}$ & $0.1 \mathrm{ppm}$ & $0.05 \mathrm{ppm}$ & $0.1 \mathrm{ppm}$ & $0.05 \mathrm{ppm}$ \\
\hline $\begin{array}{l}\text { 158-957A- } \\
3 \mathrm{X}-1,4-8\end{array}$ & 1 & 10.00 & & 300 & 47 & 69 & 2.5 & 47 & 4.4 \\
\hline $\begin{array}{l}\text { 158-957B- } \\
1 \mathrm{R}-1,3-95 \\
1 \mathrm{R}-1,3-95 \\
1 \mathrm{R}-1,3-95 \\
1 \mathrm{R}-1,3-95 \\
4 \mathrm{R}-1,0-10\end{array}$ & $\begin{array}{c}-63 \\
63-420 \\
+420 \\
+420 \\
1\end{array}$ & $\begin{array}{r}0.00 \\
0.00 \\
0.00 \\
0.00 \\
19.90\end{array}$ & & $\begin{array}{r}430 \\
580 \\
300 \\
310 \\
6\end{array}$ & $\begin{array}{l}46 \\
110 \\
100 \\
110 \\
<0.5\end{array}$ & $\begin{array}{c}160 \\
210 \\
190 \\
200 \\
0.4\end{array}$ & $\begin{array}{c}15 \\
18 \\
11 \\
12 \\
0.4\end{array}$ & $\begin{array}{c}75 \\
55 \\
41 \\
45 \\
0.8\end{array}$ & $\begin{array}{l}3.6 \\
5.0 \\
3.8 \\
3.8 \\
0.11\end{array}$ \\
\hline $\begin{array}{l}158-957 \mathrm{C}- \\
5 \mathrm{~N}-1,15-48 \\
5 \mathrm{~N}-1,15-48 \\
7 \mathrm{~N}-1,52-56 \\
7 \mathrm{~N}-2,63-69 \\
10 \mathrm{~N}-1,21-22 \\
11 \mathrm{~N}-1,58-61 \\
11 \mathrm{~N}-3,90-94 \\
12 \mathrm{~N}-1,53-59 \\
12 \mathrm{~N}-2,68-75 \\
12 \mathrm{~N}-3,86-100 \\
13 \mathrm{~N}-1,12-20 \\
14 \mathrm{~N}-1,33-41 \\
14 \mathrm{~N}-2,76.5-88 \\
15 \mathrm{~N}-1,36-40 \\
15 \mathrm{~N}-2,12-15 \\
15 \mathrm{~N}-3,132-150 \\
15 \mathrm{~N}-4,8-10 \\
16 \mathrm{~N}-1,118-125 \\
16 \mathrm{~N}-2,14-23 \\
16 \mathrm{~N}-2,94-95 \\
16 \mathrm{~N}-2,94-95\end{array}$ & $\begin{array}{c}3,5,6 \\
3,5,6 \\
6 \mathrm{~F} \\
1 \mathrm{E} \\
4 \\
3 \mathrm{D} \\
8 \mathrm{C} \\
6 \mathrm{~A} \\
9 \\
9 \\
4 \\
5 \\
6 \\
5 \\
1 \mathrm{~B} \\
11 \\
1 \\
15 \\
2 \\
9 \mathrm{~B} \\
9 \mathrm{~B}\end{array}$ & $\begin{array}{l}15.25 \\
15.25 \\
20.04 \\
21.45 \\
28.86 \\
31.18 \\
34.50 \\
35.45 \\
36.16 \\
36.94 \\
37.31 \\
40.53 \\
41.67 \\
42.50 \\
43.28 \\
45.54 \\
45.70 \\
47.39 \\
47.75 \\
48.56 \\
48.56\end{array}$ & & $\begin{array}{r}33 \\
33 \\
27 \\
22 \\
30 \\
22 \\
23 \\
21 \\
32 \\
7 \\
32 \\
17 \\
19 \\
16 \\
8 \\
14 \\
8 \\
45 \\
32 \\
7 \\
6\end{array}$ & $\begin{array}{r}0.7 \\
0.6 \\
<0.5 \\
0.7 \\
<0.5 \\
<0.5 \\
0.8 \\
0.6 \\
1.7 \\
<0.5 \\
1.0 \\
<0.5 \\
0.5 \\
0.8 \\
<0.5 \\
<0.5 \\
<0.5 \\
<0.5 \\
<0.5 \\
<0.5 \\
<0.5\end{array}$ & $\begin{array}{l}2.2 \\
2.3 \\
1.3 \\
2.2 \\
1.4 \\
0.9 \\
1.3 \\
1.2 \\
1.2 \\
0.6 \\
0.7 \\
0.4 \\
0.7 \\
1.2 \\
1.1 \\
0.7 \\
0.7 \\
1.4 \\
0.9 \\
0.5 \\
0.4\end{array}$ & $\begin{array}{c}0.32 \\
0.33 \\
0.49 \\
0.40 \\
0.24 \\
0.36 \\
0.58 \\
0.35 \\
0.72 \\
0.23 \\
0.22 \\
0.27 \\
0.13 \\
0.18 \\
<0.05 \\
0.08 \\
0.14 \\
0.37 \\
0.20 \\
<0.5 \\
<0.5\end{array}$ & $\begin{array}{r}69 \\
70 \\
72 \\
52 \\
49 \\
54 \\
100 \\
76 \\
79 \\
49 \\
85 \\
85 \\
62 \\
80 \\
19 \\
42 \\
32 \\
65 \\
52 \\
37 \\
41\end{array}$ & $\begin{array}{l}2.3 \\
2.3 \\
2.2 \\
1.3 \\
1.4 \\
1.6 \\
2.5 \\
2.8 \\
3.6 \\
0.55 \\
4.4 \\
2.1 \\
1.8 \\
1.6 \\
0.34 \\
0.79 \\
0.83 \\
3.5 \\
2.0 \\
0.49 \\
0.48\end{array}$ \\
\hline $\begin{array}{l}158-957 \mathrm{E}- \\
1 \mathrm{R}-1,23-26 \\
9 \mathrm{R}-1,16-19 \\
12 \mathrm{R}-1,16-20 \\
15 \mathrm{R}-1,27-30\end{array}$ & $\begin{array}{l}4 \\
4 \\
3 \\
6\end{array}$ & $\begin{array}{r}31.70 \\
78.16 \\
91.95 \\
106.77\end{array}$ & & $\begin{array}{r}4 \\
26 \\
11 \\
18\end{array}$ & $\begin{array}{l}<0.5 \\
<0.5 \\
<0.5 \\
<0.5\end{array}$ & $\begin{array}{l}0.3 \\
0.6 \\
1.3 \\
0.6\end{array}$ & $\begin{array}{l}0.08 \\
0.07 \\
0.34 \\
<0.5\end{array}$ & $\begin{array}{r}22 \\
118 \\
90 \\
62\end{array}$ & $\begin{array}{l}0.22 \\
1.4 \\
2.1 \\
0.94\end{array}$ \\
\hline $\begin{array}{l}158-957 \mathrm{~F}- \\
1 \mathrm{~N}-1,32-35 \\
2 \mathrm{~N}-1,11-19\end{array}$ & $\begin{array}{l}8 \\
3\end{array}$ & $\begin{array}{l}1.41 \\
5.60\end{array}$ & & $\begin{array}{l}24 \\
31\end{array}$ & $\begin{array}{r}0.7 \\
<0.5\end{array}$ & $\begin{array}{l}3.5 \\
3.0\end{array}$ & $\begin{array}{l}0.85 \\
0.72\end{array}$ & $\begin{array}{l}73 \\
69\end{array}$ & $\begin{array}{l}1.7 \\
2.0\end{array}$ \\
\hline $\begin{array}{l}158-957 \mathrm{H}- \\
1 \mathrm{~N}-1,60-73 \\
5 \mathrm{~N}-1,61-64 \\
6 \mathrm{~N}-1,14-19\end{array}$ & $\begin{array}{c}13 \\
6 \\
3\end{array}$ & $\begin{array}{r}9.30 \\
27.30 \\
31.34\end{array}$ & & $\begin{array}{l}11 \\
13 \\
14\end{array}$ & $\begin{array}{r}<0.5 \\
<0.5 \\
0.6\end{array}$ & $\begin{array}{l}4.6 \\
1.0 \\
5.8\end{array}$ & $\begin{array}{l}1.9 \\
0.32 \\
1.8\end{array}$ & $\begin{array}{l}43 \\
60 \\
51\end{array}$ & $\begin{array}{l}0.78 \\
2.7 \\
0.93\end{array}$ \\
\hline $\begin{array}{l}\text { 158-957I- } \\
1 \mathrm{~N}-1,33-41\end{array}$ & 6 & 9.33 & & 150 & 55 & 19 & 0.50 & 170 & 20 \\
\hline $\begin{array}{l}158-957 \mathrm{~K}- \\
1 \mathrm{X}-1,24-29 \\
1 \mathrm{~N}-1,37-41 \\
3 \mathrm{X}-1,36-38\end{array}$ & $\begin{array}{l}4 \\
9 \\
7\end{array}$ & $\begin{array}{r}0.21 \\
10.37 \\
14.82\end{array}$ & & $\begin{array}{l}270 \\
170 \\
230\end{array}$ & $\begin{array}{r}70 \\
18 \\
200\end{array}$ & $\begin{array}{l}24 \\
18 \\
19\end{array}$ & $\begin{array}{l}0.09 \\
1.2 \\
2.1\end{array}$ & $\begin{array}{r}110 \\
100 \\
86\end{array}$ & $\begin{array}{l}30 \\
18 \\
17\end{array}$ \\
\hline $\begin{array}{l}\text { 158-957M- } \\
\text { 1R-1, 16-47 } \\
\text { 1R-1, 16-47 } \\
\text { 1R-1, 16-47 } \\
\text { 1R-2, 8-12 } \\
\text { 2R-1, 2-5 } \\
\text { 3R-1, 57-60 } \\
\text { 3R-1, 123-125 } \\
\text { 3R-2, 1-4 } \\
\text { 5R-1, 90-92 } \\
\text { 7R-1, 0-4 } \\
\text { 8R-1, 0-4 }\end{array}$ & $\begin{array}{c}-63 \\
420-63 \\
+420 \\
3 \\
1 \\
14 \\
28 \\
1 \\
15 \\
1 \\
1\end{array}$ & $\begin{array}{r}0.00 \\
0.00 \\
0.00 \\
0.63 \\
9.30 \\
14.87 \\
15.49 \\
15.80 \\
25.18 \\
34.30 \\
38.30\end{array}$ & & $\begin{array}{r}160 \\
130 \\
65 \\
71 \\
83 \\
150 \\
54 \\
32 \\
22 \\
16 \\
83\end{array}$ & $\begin{array}{c}5.8 \\
1.0 \\
0.8 \\
0.3 \\
4.3 \\
56 \\
27 \\
0.8 \\
0.7 \\
2.2 \\
12\end{array}$ & $\begin{array}{c}4.1 \\
1.0 \\
0.5 \\
2.4 \\
22 \\
20 \\
11 \\
1.4 \\
9.3 \\
0.5 \\
7.5\end{array}$ & $\begin{array}{l}0.30 \\
0.11 \\
<0.5 \\
0.19 \\
4.4 \\
0.33 \\
0.53 \\
1.0 \\
0.68 \\
0.38 \\
1.5\end{array}$ & $\begin{array}{l}39 \\
32 \\
30 \\
17 \\
71 \\
93 \\
89 \\
76 \\
37 \\
12 \\
97\end{array}$ & $\begin{array}{l}1.6 \\
0.39 \\
0.19 \\
2.7 \\
3.2 \\
10 \\
3.7 \\
2.4 \\
1.0 \\
0.53 \\
3.7\end{array}$ \\
\hline $\begin{array}{l}\text { 158-957O- } \\
\text { 2R-1, 22-24 } \\
\text { 4R-1, 45-50 }\end{array}$ & $\begin{array}{l}5 \\
9\end{array}$ & $\begin{array}{r}8.10 \\
16.35\end{array}$ & & $\begin{array}{l}21 \\
36\end{array}$ & $\begin{array}{l}0.5 \\
0.6\end{array}$ & $\begin{array}{l}0.9 \\
1.3\end{array}$ & $\begin{array}{l}0.44 \\
1.1\end{array}$ & $\begin{array}{l}70 \\
86\end{array}$ & $\begin{array}{l}1.4 \\
2.3\end{array}$ \\
\hline $\begin{array}{l}\text { 158-957P- } \\
\text { 1R-1, 44-46 } \\
5 \mathrm{R}-1,9-13 \\
5 \mathrm{R}-1,9-13 \\
\text { 9R-1, 11-13 } \\
10 \mathrm{R}-1,1-3 \\
12 \mathrm{R}-1,15-100 \\
12 \mathrm{R}-1,15-100 \\
12 \mathrm{R}-1,15-100 \\
\text { 12R-2, 25-28 } \\
\text { 12R-4, 53-57 } \\
13 \mathrm{~W}-1,50-58 \\
13 \mathrm{~W}-1+2,0-150\end{array}$ & $\begin{array}{c}8 \\
2 \\
2 \\
2 \\
1 \\
-63 \\
420-63 \\
+420 \\
6 \\
5 \\
- \\
-\end{array}$ & $\begin{array}{r}0.43 \\
21.57 \\
21.57 \\
40.19 \\
45.10 \\
54.40 \\
54.40 \\
54.40 \\
55.89 \\
57.61 \\
15.00 \\
15.00\end{array}$ & & $\begin{array}{l}11 \\
24 \\
22 \\
27 \\
20 \\
93 \\
77 \\
57 \\
49 \\
15 \\
45 \\
51\end{array}$ & $\begin{array}{r}<0.5 \\
0.7 \\
0.8 \\
0.5 \\
<0.5 \\
16 \\
11 \\
8.8 \\
<0.5 \\
<0.5 \\
4.2 \\
8.8\end{array}$ & $\begin{array}{c}0.6 \\
0.6 \\
0.6 \\
1.2 \\
1.7 \\
11 \\
8.7 \\
7.4 \\
0.4 \\
2.0 \\
5.8 \\
5.4\end{array}$ & $\begin{array}{c}0.39 \\
<0.5 \\
0.09 \\
0.19 \\
0.41 \\
4.7 \\
3.2 \\
2.5 \\
<0.5 \\
0.73 \\
1.9 \\
2.4\end{array}$ & $\begin{array}{r}54 \\
90 \\
94 \\
92 \\
84 \\
100 \\
87 \\
82 \\
29 \\
210 \\
94 \\
91\end{array}$ & $\begin{array}{l}0.84 \\
4.4 \\
4.4 \\
2.0 \\
2.7 \\
3.7 \\
3.9 \\
3.7 \\
2.3 \\
1.6 \\
4.2 \\
3.4\end{array}$ \\
\hline $\begin{array}{l}\text { 158-957Q- } \\
\text { 1R-1, 13-18 } \\
\text { 1R-3, 70-100 } \\
\text { 1R-3, 119-121 } \\
\text { 2R-1, 0-4 }\end{array}$ & $\frac{-}{1}$ & $\begin{array}{l}0.13 \\
3.75 \\
4.10 \\
9.50\end{array}$ & & $\begin{array}{r}150 \\
44 \\
12 \\
29\end{array}$ & $\begin{array}{c}24 \\
13 \\
21 \\
2.5\end{array}$ & $\begin{array}{r}4.8 \\
4.4 \\
3.3 \\
24\end{array}$ & $\begin{array}{l}2.8 \\
2.6 \\
2.9 \\
0.16\end{array}$ & $\begin{array}{c}30 \\
54 \\
8.1 \\
15\end{array}$ & $\begin{array}{l}2.1 \\
2.0 \\
0.60 \\
0.18\end{array}$ \\
\hline
\end{tabular}

Note: $\mathrm{ND}=$ element not determined; $<=$ indicates values below reported detection. 
Table 3 (continued).

\begin{tabular}{|c|c|c|c|c|c|c|c|c|c|c|c|c|}
\hline & & & $\begin{array}{l}\text { Element: } \\
\text { Method: }\end{array}$ & $\begin{array}{c}\mathrm{Ga} \\
\text { ICP-MS }\end{array}$ & $\begin{array}{c}\mathrm{Rb} \\
\mathrm{ICP}-\mathrm{MS}\end{array}$ & $\begin{array}{c}\text { Cs } \\
\text { ICP-MS }\end{array}$ & $\begin{array}{c}\mathrm{Nb} \\
\text { ICP-MS }\end{array}$ & $\begin{array}{c}\text { Ta } \\
\text { ICP-MS }\end{array}$ & $\begin{array}{c}\mathrm{Zr} \\
\text { ICP-MS }\end{array}$ & $\begin{array}{c}\mathrm{Hf} \\
\text { ICP-MS }\end{array}$ & $\begin{array}{c}\text { U } \\
\text { ICP-MS }\end{array}$ & $\begin{array}{c}\text { Th } \\
\text { ICP-MS }\end{array}$ \\
\hline $\begin{array}{l}\text { Core, section, } \\
\text { interval }(\mathrm{cm})\end{array}$ & Piece & $\begin{array}{l}\text { Depth } \\
\text { (mbsf) }\end{array}$ & $\begin{array}{l}\text { Detection } \\
\text { limit: }\end{array}$ & $0.1 \mathrm{ppm}$ & $0.05 \mathrm{ppm}$ & $0.02 \mathrm{ppm}$ & $0.05 \mathrm{ppm}$ & $0.2 \mathrm{ppm}$ & $0.5 \mathrm{ppm}$ & $0.05 \mathrm{ppm}$ & $0.2 \mathrm{ppm}$ & $0.02 \mathrm{ppm}$ \\
\hline $\begin{array}{r}158-957 \mathrm{~A}- \\
3 \mathrm{X}-1,4-8\end{array}$ & 1 & 10.00 & & 46 & 0.81 & 0.04 & 0.39 & $<0.2$ & 6.4 & 0.10 & 10 & 0.18 \\
\hline $\begin{array}{c}\text { 158-957B- } \\
\text { 1R-1,3-95 } \\
\text { 1R-1, 3-95 } \\
\text { 1R-1, 3-95 } \\
\text { 1R-1, 3-95 } \\
\text { 4R-1, 0-10 }\end{array}$ & $\begin{array}{c}-63 \\
63-420 \\
+420 \\
+420 \\
1\end{array}$ & $\begin{array}{r}0.00 \\
0.00 \\
0.00 \\
0.00 \\
19.90\end{array}$ & & $\begin{array}{l}58 \\
78 \\
62 \\
61 \\
18\end{array}$ & $\begin{array}{l}0.67 \\
0.37 \\
0.37 \\
0.40 \\
0.27\end{array}$ & $\begin{array}{r}0.03 \\
<0.02 \\
0.02 \\
0.02 \\
<0.02\end{array}$ & $\begin{array}{l}0.57 \\
0.26 \\
0.20 \\
0.21 \\
3.20\end{array}$ & $\begin{array}{r}0.2 \\
<0.2 \\
0.3 \\
<0.2 \\
0.2\end{array}$ & $\begin{array}{c}12 \\
5.9 \\
3.8 \\
4.0 \\
<100\end{array}$ & $\begin{array}{l}0.21 \\
0.11 \\
0.06 \\
0.06 \\
3.40\end{array}$ & $\begin{array}{c}30 \\
17 \\
13 \\
12 \\
8.5\end{array}$ & $\begin{array}{l}0.32 \\
0.14 \\
0.10 \\
0.11 \\
0.20\end{array}$ \\
\hline $\begin{array}{l}\text { 158-957C- } \\
\text { 5N-1, 15-48 } \\
5 \mathrm{~N}-1,15-48 \\
7 \mathrm{~N}-1,52-56 \\
7 \mathrm{~N}-2,63-69 \\
10 \mathrm{~N}-1,21-22 \\
11 \mathrm{~N}-1,58-61 \\
11 \mathrm{~N}-3,90-94 \\
12 \mathrm{~N}-1,53-59 \\
12 \mathrm{~N}-2,68-75 \\
12 \mathrm{~N}-3,86-100 \\
13 \mathrm{~N}-1,12-20 \\
14 \mathrm{~N}-1,33-41 \\
14 \mathrm{~N}-2,76.5-88 \\
15 \mathrm{~N}-1,36-40 \\
15 \mathrm{~N}-2,12-15 \\
15 \mathrm{~N}-3,132-150 \\
15 \mathrm{~N}-4,8-10 \\
16 \mathrm{~N}-1,118-125 \\
16 \mathrm{~N}-2,14-23 \\
16 \mathrm{~N}-2,94-95 \\
16 \mathrm{~N}-2,94-95\end{array}$ & $\begin{array}{c}3,5,6 \\
3,5,6 \\
6 \mathrm{~F} \\
1 \mathrm{E} \\
4 \\
3 \mathrm{D} \\
8 \mathrm{C} \\
6 \mathrm{~A} \\
9 \\
9 \\
4 \\
5 \\
6 \\
5 \\
1 \mathrm{~B} \\
11 \\
1 \\
15 \\
2 \\
9 \mathrm{~B} \\
9 \mathrm{~B}\end{array}$ & $\begin{array}{l}15.25 \\
15.25 \\
20.04 \\
21.45 \\
28.86 \\
31.18 \\
34.50 \\
35.45 \\
36.16 \\
36.94 \\
37.31 \\
40.53 \\
41.67 \\
42.50 \\
43.28 \\
45.54 \\
45.70 \\
47.39 \\
47.75 \\
48.56 \\
48.56\end{array}$ & & $\begin{array}{l}1.6 \\
1.5 \\
1.6 \\
3.0 \\
1.7 \\
2.5 \\
4.1 \\
1.7 \\
2.7 \\
2.8 \\
1.5 \\
1.1 \\
0.8 \\
1.2 \\
0.7 \\
0.7 \\
1.1 \\
3.1 \\
2.3 \\
0.6 \\
0.7\end{array}$ & $\begin{array}{r}<0.05 \\
0.06 \\
<0.05 \\
0.05 \\
0.11 \\
0.06 \\
<0.05 \\
<0.05 \\
<0.05 \\
0.06 \\
<0.05 \\
0.08 \\
<0.05 \\
0.06 \\
0.13 \\
0.06 \\
0.07 \\
0.06 \\
0.05 \\
0.07 \\
<0.05\end{array}$ & $\begin{array}{l}<0.02 \\
<0.02 \\
<0.02 \\
<0.02 \\
<0.02 \\
<0.02 \\
<0.02 \\
<0.02 \\
<0.02 \\
<0.02 \\
<0.02 \\
<0.02 \\
<0.02 \\
<0.02 \\
<0.02 \\
<0.02 \\
<0.02 \\
<0.02 \\
<0.02 \\
<0.02 \\
<0.02\end{array}$ & $\begin{array}{r}0.06 \\
0.06 \\
<0.05 \\
0.06 \\
0.06 \\
<0.05 \\
<0.05 \\
0.10 \\
<0.05 \\
0.09 \\
0.05 \\
<0.05 \\
<0.05 \\
0.06 \\
0.15 \\
<0.05 \\
0.07 \\
<0.05 \\
0.10 \\
<0.05 \\
0.05\end{array}$ & $\begin{array}{r}0.7 \\
0.5 \\
0.4 \\
0.2 \\
0.2 \\
0.3 \\
0.3 \\
<0.2 \\
<0.2 \\
<0.2 \\
<0.2 \\
<0.2 \\
<0.2 \\
<0.2 \\
<0.2 \\
0.2 \\
<0.2 \\
<0.2 \\
1.9 \\
<0.2 \\
<0.2\end{array}$ & $\begin{array}{r}1.2 \\
1.4 \\
0.6 \\
0.8 \\
0.9 \\
0.7 \\
0.7 \\
2.0 \\
<0.5 \\
2.2 \\
0.7 \\
<0.5 \\
0.7 \\
1.2 \\
4.4 \\
0.8 \\
1.9 \\
<0.5 \\
4.8 \\
<0.5 \\
1.2\end{array}$ & $\begin{array}{r}<0.05 \\
<0.05 \\
<0.05 \\
<0.05 \\
<0.05 \\
<0.05 \\
<0.05 \\
0.05 \\
<0.05 \\
0.05 \\
<0.05 \\
<0.05 \\
<0.05 \\
<0.05 \\
0.12 \\
<0.05 \\
0.05 \\
<0.05 \\
0.15 \\
<0.05 \\
<0.05\end{array}$ & $\begin{array}{c}16 \\
16 \\
9.5 \\
8.6 \\
12 \\
10 \\
6.2 \\
2.8 \\
4.5 \\
9.9 \\
2.5 \\
2.0 \\
4.5 \\
6.5 \\
2.4 \\
2.3 \\
2.0 \\
5.6 \\
13 \\
2.3 \\
2.2\end{array}$ & $\begin{array}{l}<0.02 \\
<0.02 \\
<0.02 \\
<0.02 \\
<0.02 \\
<0.02 \\
<0.02 \\
<0.02 \\
<0.02 \\
<0.02 \\
<0.02 \\
<0.02 \\
<0.02 \\
<0.02 \\
<0.02 \\
<0.02 \\
<0.02 \\
<0.02 \\
<0.02 \\
<0.02 \\
<0.02\end{array}$ \\
\hline $\begin{array}{c}158-957 \mathrm{E}- \\
1 \mathrm{R}-1,23-26 \\
9 \mathrm{R}-1,16-19 \\
12 \mathrm{R}-1,16-20 \\
15 \mathrm{R}-1,27-30\end{array}$ & $\begin{array}{l}4 \\
4 \\
3 \\
6\end{array}$ & $\begin{array}{r}31.70 \\
78.16 \\
91.95 \\
106.77\end{array}$ & & $\begin{array}{l}0.5 \\
0.8 \\
2.0 \\
0.4\end{array}$ & $\begin{array}{r}0.06 \\
0.06 \\
0.06 \\
<0.05\end{array}$ & $\begin{array}{l}<0.02 \\
<0.02 \\
<0.02 \\
<0.02\end{array}$ & $\begin{array}{r}<0.05 \\
0.07 \\
0.06 \\
<0.05\end{array}$ & $\begin{array}{r}<0.2 \\
<0.2 \\
<0.2 \\
0.2\end{array}$ & $\begin{array}{r}<0.5 \\
1.4 \\
1.5 \\
1.3\end{array}$ & $\begin{array}{l}<0.05 \\
<0.05 \\
<0.05 \\
<0.05\end{array}$ & $\begin{array}{l}2.0 \\
2.8 \\
5.2 \\
3.6\end{array}$ & $\begin{array}{l}<0.02 \\
<0.02 \\
<0.02 \\
<0.02\end{array}$ \\
\hline $\begin{array}{l}158-957 \mathrm{~F}- \\
1 \mathrm{~N}-1,32-35 \\
2 \mathrm{~N}-1,11-19\end{array}$ & $\begin{array}{l}8 \\
3\end{array}$ & $\begin{array}{l}1.41 \\
5.60\end{array}$ & & $\begin{array}{l}2.4 \\
2.9\end{array}$ & $\begin{array}{r}0.06 \\
<0.05\end{array}$ & $\begin{array}{l}<0.02 \\
<0.02\end{array}$ & $\begin{array}{l}<0.05 \\
<0.05\end{array}$ & $\begin{array}{r}1.7 \\
<0.2\end{array}$ & $\begin{array}{r}1.2 \\
<0.5\end{array}$ & $\begin{array}{l}<0.05 \\
<0.05\end{array}$ & $\begin{array}{l}7.1 \\
20\end{array}$ & $\begin{array}{l}<0.02 \\
<0.02\end{array}$ \\
\hline $\begin{array}{l}158-957 \mathrm{H}- \\
1 \mathrm{~N}-1,60-73 \\
5 \mathrm{~N}-1,61-64 \\
6 \mathrm{~N}-1,14-19\end{array}$ & $\begin{array}{c}13 \\
6 \\
3\end{array}$ & $\begin{array}{r}9.30 \\
27.30 \\
31.34\end{array}$ & & $\begin{array}{l}3.3 \\
1.7 \\
2.8\end{array}$ & $\begin{array}{l}0.06 \\
0.09 \\
0.08\end{array}$ & $\begin{array}{l}<0.02 \\
<0.02 \\
<0.02\end{array}$ & $\begin{array}{r}<0.05 \\
0.10 \\
0.06\end{array}$ & $\begin{array}{r}<0.2 \\
0.3 \\
<0.2\end{array}$ & $\begin{array}{r}<0.5 \\
2.1 \\
2.5\end{array}$ & $\begin{array}{r}<0.05 \\
<0.05 \\
0.05\end{array}$ & $\begin{array}{l}5.7 \\
3.7 \\
7.7\end{array}$ & $\begin{array}{l}<0.02 \\
<0.02 \\
<0.02\end{array}$ \\
\hline $\begin{array}{l}158-957 \mathrm{I}- \\
1 \mathrm{~N}-1,33-41\end{array}$ & 6 & 9.33 & & 16 & 0.17 & 0.05 & $<0.05$ & $<0.2$ & $<0.5$ & $<0.05$ & 1.5 & $<0.02$ \\
\hline $\begin{array}{l}158-957 \mathrm{~K}- \\
1 \mathrm{X}-1,24-29 \\
1 \mathrm{~N}-1,37-41 \\
3 \mathrm{X}-1,36-38\end{array}$ & $\begin{array}{l}4 \\
9 \\
7\end{array}$ & $\begin{array}{r}0.21 \\
10.37 \\
14.82\end{array}$ & & $\begin{array}{l}23 \\
15 \\
95\end{array}$ & $\begin{array}{l}0.10 \\
0.05 \\
0.06\end{array}$ & $\begin{array}{l}<0.02 \\
<0.02 \\
<0.02\end{array}$ & $\begin{array}{r}0.05 \\
<0.05 \\
0.06\end{array}$ & $\begin{array}{l}<0.2 \\
<0.2 \\
<0.2\end{array}$ & $\begin{array}{l}<0.5 \\
<0.5 \\
<0.5\end{array}$ & $\begin{array}{l}<0.05 \\
<0.05 \\
<0.05\end{array}$ & $\begin{array}{c}1.9 \\
12 \\
9.6\end{array}$ & $\begin{array}{l}<0.02 \\
<0.02 \\
<0.02\end{array}$ \\
\hline $\begin{array}{l}\text { 158-957M- } \\
1 \mathrm{R}-1,16-47 \\
1 \mathrm{R}-1,16-47 \\
1 \mathrm{R}-1,16-47 \\
1 \mathrm{R}-2,8-12 \\
\text { 2R-1, }-5 \\
\text { 3R-1, 57-60 } \\
\text { 3R-1, 123-125 } \\
\text { 3R-2, 1-4 } \\
\text { 5R-1, 90-92 } \\
\text { 7R-1, } 0-4 \\
\text { 8R-1, }-4\end{array}$ & $\begin{array}{c}-63 \\
420-63 \\
+420 \\
3 \\
1 \\
14 \\
28 \\
1 \\
15 \\
1 \\
1\end{array}$ & $\begin{array}{r}0.00 \\
0.00 \\
0.00 \\
0.63 \\
9.30 \\
14.87 \\
15.49 \\
15.80 \\
25.18 \\
34.30 \\
38.30\end{array}$ & & $\begin{array}{c}4.5 \\
3.0 \\
2.3 \\
0.5 \\
22 \\
8.8 \\
9.4 \\
3.7 \\
3.9 \\
0.9 \\
3.2\end{array}$ & $\begin{array}{c}0.86 \\
0.76 \\
0.74 \\
0.10 \\
0.06 \\
<0.05 \\
0.05 \\
0.06 \\
0.08 \\
0.07 \\
0.06\end{array}$ & $\begin{array}{r}0.03 \\
0.02 \\
<0.02 \\
<0.02 \\
<0.02 \\
<0.02 \\
<0.02 \\
<0.02 \\
<0.02 \\
<0.02 \\
<0.02\end{array}$ & $\begin{array}{l}0.26 \\
0.16 \\
0.12 \\
0.09 \\
0.13 \\
0.06 \\
0.07 \\
0.06 \\
0.12 \\
0.22 \\
0.06\end{array}$ & $\begin{array}{r}0.2 \\
0.2 \\
<0.2 \\
<0.2 \\
<0.2 \\
<0.2 \\
<0.2 \\
<0.2 \\
<0.2 \\
<0.2 \\
<0.2\end{array}$ & $\begin{array}{c}16 \\
11 \\
13 \\
0.8 \\
0.3 \\
<0.5 \\
0.6 \\
<0.5 \\
2.2 \\
0.6 \\
0.6\end{array}$ & $\begin{array}{r}0.32 \\
0.20 \\
0.27 \\
<0.02 \\
<0.02 \\
<0.05 \\
<0.05 \\
<0.05 \\
0.05 \\
<0.02 \\
<0.05\end{array}$ & $\begin{array}{c}11 \\
11 \\
10 \\
2.5 \\
11 \\
9.0 \\
13 \\
12 \\
12 \\
2.6 \\
39\end{array}$ & $\begin{array}{l}0.12 \\
0.05 \\
0.03 \\
<0.02 \\
<0.02 \\
<0.02 \\
<0.02 \\
<0.02 \\
<0.02 \\
<0.02 \\
<0.02\end{array}$ \\
\hline $\begin{array}{l}158-957 \mathrm{O}- \\
2 \mathrm{R}-1,22-24 \\
4 \mathrm{R}-1,45-50\end{array}$ & $\begin{array}{l}5 \\
9\end{array}$ & $\begin{array}{r}8.10 \\
16.35\end{array}$ & & $\begin{array}{l}2.1 \\
6.0\end{array}$ & $\begin{array}{r}<0.05 \\
0.06\end{array}$ & $\begin{array}{l}<0.02 \\
<0.02\end{array}$ & $\begin{array}{l}<0.05 \\
<0.05\end{array}$ & $\begin{array}{r}<0.2 \\
0.2\end{array}$ & $\begin{array}{l}<0.5 \\
<0.5\end{array}$ & $\begin{array}{l}<0.05 \\
<0.05\end{array}$ & $\begin{array}{c}6.0 \\
12\end{array}$ & $\begin{array}{l}<0.02 \\
<0.02\end{array}$ \\
\hline $\begin{array}{l}\text { 158-957P- } \\
1 \mathrm{R}-1,44-46 \\
5 \mathrm{R}-1,9-13 \\
5 \mathrm{R}-1,9-13 \\
\text { 9R-1, } 11-13 \\
10 \mathrm{R}-1,1-3 \\
12 \mathrm{R}-1,15-100 \\
12 \mathrm{R}-1,15-100 \\
12 \mathrm{R}-1,15-100 \\
12 \mathrm{R}-2,25-28 \\
12 \mathrm{R}-4,53-57 \\
13 \mathrm{~W}-1,50-58 \\
13 \mathrm{~W}-1+2,0-150\end{array}$ & $\begin{array}{c}8 \\
2 \\
2 \\
2 \\
1 \\
-63 \\
420-63 \\
+420 \\
6 \\
5 \\
- \\
-\end{array}$ & $\begin{array}{r}0.43 \\
21.57 \\
21.57 \\
40.19 \\
45.10 \\
54.40 \\
54.40 \\
54.40 \\
55.89 \\
57.61 \\
15.00 \\
15.00\end{array}$ & & $\begin{array}{c}1.5 \\
0.5 \\
0.6 \\
1.1 \\
1.5 \\
17 \\
12 \\
9.8 \\
1.2 \\
3.0 \\
6.7 \\
8.9\end{array}$ & $\begin{array}{r}0.08 \\
<0.05 \\
<0.05 \\
<0.05 \\
<0.05 \\
0.14 \\
0.14 \\
0.11 \\
0.10 \\
0.08 \\
0.10 \\
0.09\end{array}$ & $\begin{array}{l}<0.02 \\
<0.02 \\
<0.02 \\
<0.02 \\
<0.02 \\
<0.02 \\
<0.02 \\
<0.02 \\
<0.02 \\
<0.02 \\
<0.02 \\
<0.02\end{array}$ & $\begin{array}{r}<0.05 \\
<0.05 \\
0.05 \\
<0.05 \\
<0.05 \\
0.07 \\
0.06 \\
0.08 \\
0.19 \\
0.06 \\
0.16 \\
0.14\end{array}$ & $\begin{array}{r}<0.2 \\
<0.2 \\
<0.2 \\
<0.2 \\
0.2 \\
<0.2 \\
<0.2 \\
<0.2 \\
<0.2 \\
<0.2 \\
0.4 \\
<0.2\end{array}$ & $\begin{array}{l}14 \\
<0.5 \\
<0.5 \\
<0.5 \\
<0.5 \\
1.3 \\
0.7 \\
1.7 \\
7.9 \\
3.9 \\
0.5 \\
1.0\end{array}$ & $\begin{array}{r}0.27 \\
<0.05 \\
<0.05 \\
<0.05 \\
<0.05 \\
<0.05 \\
<0.05 \\
<0.05 \\
0.21 \\
0.08 \\
<0.05 \\
0.02\end{array}$ & $\begin{array}{c}2.4 \\
2.9 \\
2.8 \\
14 \\
21 \\
6.1 \\
5.5 \\
5.9 \\
3.9 \\
24 \\
5.6 \\
5.0\end{array}$ & $\begin{array}{r}<0.02 \\
<0.02 \\
<0.02 \\
<0.02 \\
<0.02 \\
0.03 \\
0.02 \\
<0.02 \\
<0.02 \\
<0.02 \\
0.02 \\
<0.02\end{array}$ \\
\hline $\begin{array}{l}\text { 158-957Q- } \\
\text { 1R-1, 13-18 } \\
\text { 1R-3, 70-100 } \\
\text { 1R-3, 119-121 } \\
\text { 2R-1, 0-4 }\end{array}$ & $\bar{z}$ & $\begin{array}{l}0.13 \\
3.75 \\
4.10 \\
9.50\end{array}$ & & $\begin{array}{c}21 \\
7.7 \\
5.4 \\
14\end{array}$ & $\begin{array}{l}0.22 \\
0.10 \\
0.12 \\
0.08\end{array}$ & $\begin{array}{l}<0.02 \\
<0.02 \\
<0.02 \\
<0.02\end{array}$ & $\begin{array}{l}0.12 \\
0.06 \\
0.07 \\
0.14\end{array}$ & $\begin{array}{l}<0.2 \\
<0.2 \\
<0.2 \\
<0.2\end{array}$ & $\begin{array}{r}1.8 \\
<0.5 \\
1.0 \\
0.6\end{array}$ & $\begin{array}{l}0.03 \\
<0.05 \\
<0.02 \\
<0.02\end{array}$ & $\begin{array}{l}7.1 \\
7.3 \\
7.2 \\
2.5\end{array}$ & $\begin{array}{l}0.03 \\
<0.02 \\
<0.02 \\
<0.02\end{array}$ \\
\hline
\end{tabular}


Table 4. Trace element composition of samples from Site 957.

\begin{tabular}{|c|c|c|c|c|c|c|c|c|c|c|c|c|c|c|c|c|c|c|}
\hline $\begin{array}{l}\text { Core, section, } \\
\text { interval }(\mathrm{cm})\end{array}$ & Piece & $\begin{array}{l}\text { Depth } \\
\text { (mbsf) }\end{array}$ & $\begin{array}{l}\text { Element: } \\
\text { Method: } \\
\text { Detection } \\
\text { limit: }\end{array}$ & $\begin{array}{c}\mathrm{Au} \\
\text { INAA }\end{array}$ & $\begin{array}{c}\mathrm{Ag} \\
\text { INAA }\end{array}$ & $\begin{array}{c}\text { As } \\
\text { INAA }\end{array}$ & $\begin{array}{c}\mathrm{Sb} \\
\text { INAA }\end{array}$ & $\begin{array}{c}\text { Mo } \\
\text { INAA } \\
5 \mathrm{ppm}\end{array}$ & $\begin{array}{c}\text { Co } \\
\text { INAA }\end{array}$ & $\begin{array}{c}\mathrm{Se} \\
\text { INAA }\end{array}$ & $\begin{array}{c}\mathrm{Fe} \\
\text { INAA }\end{array}$ & $\begin{array}{c}\mathrm{Zn} \\
\text { INAA } \\
50 \mathrm{ppm}\end{array}$ & $\begin{array}{c}\mathrm{Hg} \\
\text { INAA }\end{array}$ & $\begin{array}{c}\mathrm{Sn} \\
\text { INAA } \\
0.01 \%\end{array}$ & $\begin{array}{c}\text { W } \\
\text { INAA }\end{array}$ & $\begin{array}{c}\mathrm{Br} \\
\text { INAA } \\
1 \mathrm{ppm}\end{array}$ & $\begin{array}{c}\mathrm{Ir} \\
\text { INAA } \\
5 \mathrm{ppm}\end{array}$ & $\begin{array}{c}\mathrm{U} \\
\text { INAA } \\
0.5 \mathrm{ppm}\end{array}$ \\
\hline $\begin{array}{r}158-957 \mathrm{~A}- \\
3 \mathrm{X}-1,4-8\end{array}$ & 1 & 10.00 & & 3200 & 66 & 55 & 12 & 40 & 13 & 5 & 20.5 & 30000 & 12 & $<0.04$ & $<4$ & 35 & $<7$ & 8.7 \\
\hline $\begin{array}{l}\text { 158-957B- } \\
\text { 1R-1, 3-95 } \\
1 \mathrm{R}-1,3-95 \\
1 \mathrm{R}-1,3-95 \\
1 \mathrm{R}-1,3-95 \\
4 \mathrm{R}-1,0-10\end{array}$ & $\begin{array}{c}-63 \\
420-63 \\
+420 \\
+420 \\
1\end{array}$ & $\begin{array}{r}0.00 \\
0.00 \\
0.00 \\
0.00 \\
19.90\end{array}$ & & $\begin{array}{l}3200 \\
\text { ND } \\
\text { ND } \\
\text { ND } \\
9\end{array}$ & $\begin{array}{l}170 \\
\text { ND } \\
\text { ND } \\
\text { ND } \\
<5\end{array}$ & $\begin{array}{l}60 \\
\text { ND } \\
\text { ND } \\
\text { ND } \\
<2\end{array}$ & $\begin{array}{l}16 \\
\text { ND } \\
\text { ND } \\
\text { ND } \\
<0.2\end{array}$ & $\begin{array}{r}79 \\
\text { ND } \\
\text { ND } \\
\text { ND } \\
5\end{array}$ & $\begin{array}{r}51 \\
\text { ND } \\
\text { ND } \\
\text { ND } \\
49\end{array}$ & $\begin{array}{l}<5 \\
\text { ND } \\
\text { ND } \\
\text { ND } \\
<51\end{array}$ & $\begin{array}{l}29.4 \\
\text { ND } \\
\text { ND } \\
\text { ND } \\
2.2\end{array}$ & $\begin{array}{r}24000 \\
\text { ND } \\
\text { ND } \\
\text { ND } \\
260\end{array}$ & $\begin{array}{l}<1 \\
\text { ND } \\
\text { ND } \\
\text { ND } \\
<1\end{array}$ & $\begin{array}{l}<0.03 \\
\text { ND } \\
\text { ND } \\
\text { ND } \\
<0.03\end{array}$ & $\begin{array}{l}<4 \\
\text { ND } \\
\text { ND } \\
\text { ND } \\
<4\end{array}$ & $\begin{array}{r}32 \\
\text { ND } \\
\text { ND } \\
\text { ND } \\
14\end{array}$ & $\begin{array}{l}<6 \\
\text { ND } \\
\text { ND } \\
\text { ND } \\
<5\end{array}$ & $\begin{array}{l}12 \\
\text { ND } \\
\text { ND } \\
\text { ND } \\
10\end{array}$ \\
\hline $\begin{array}{l}158-957 \mathrm{C}- \\
5 \mathrm{~N}-1,15-48 \\
5 \mathrm{~N}-1,15-48 \\
7 \mathrm{~N}-1,52-56 \\
7 \mathrm{~N}-2,63-69 \\
10 \mathrm{~N}-1,21-22 \\
11 \mathrm{~N}-1,58-61 \\
11 \mathrm{~N}-3,90-94 \\
12 \mathrm{~N}-1,53-59 \\
12 \mathrm{~N}-2,68-75 \\
12 \mathrm{~N}-3,86-100 \\
13 \mathrm{~N}-1,12-20 \\
14 \mathrm{~N}-1,33-41 \\
14 \mathrm{~N}-2,76.5-88 \\
15 \mathrm{~N}-1,36-40 \\
15 \mathrm{~N}-2,12-15 \\
15 \mathrm{~N}-3,132-150 \\
15 \mathrm{~N}-4,8-10 \\
16 \mathrm{~N}-1,118-125 \\
16 \mathrm{~N}-2,14-23 \\
16 \mathrm{~N}-2,94-95 \\
16 \mathrm{~N}-2,94-95\end{array}$ & $\begin{array}{c}3,5,6 \\
3,5,6 \\
6 \mathrm{~F} \\
1 \mathrm{E} \\
4 \\
3 \mathrm{D} \\
8 \mathrm{C} \\
6 \mathrm{~A} \\
9 \\
9 \\
4 \\
5 \\
6 \\
5 \\
1 \mathrm{~B} \\
11 \\
1 \\
15 \\
2 \\
9 \mathrm{~B} \\
9 \mathrm{~B}\end{array}$ & $\begin{array}{l}15.25 \\
15.25 \\
20.04 \\
21.45 \\
28.86 \\
31.18 \\
34.50 \\
35.45 \\
36.16 \\
36.94 \\
37.31 \\
40.53 \\
41.67 \\
42.50 \\
43.28 \\
45.54 \\
45.70 \\
47.39 \\
47.75 \\
48.56 \\
48.56\end{array}$ & & $\begin{array}{c}445 \\
\text { ND } \\
195 \\
93 \\
138 \\
131 \\
235 \\
119 \\
234 \\
<5 \\
191 \\
66 \\
157 \\
179 \\
23 \\
29 \\
17 \\
161 \\
114 \\
81 \\
\text { ND }\end{array}$ & $\begin{array}{r}<5 \\
\text { ND } \\
<5 \\
<5 \\
<5 \\
<5 \\
<5 \\
<5 \\
<5 \\
<5 \\
<5 \\
<5 \\
<5 \\
<5 \\
<5 \\
<5 \\
<5 \\
<5 \\
<5 \\
<5 \\
<5 \\
<5 \\
\text { ND }\end{array}$ & $\begin{array}{r}51 \\
\text { ND } \\
37 \\
33 \\
36 \\
27 \\
46 \\
36 \\
57 \\
26 \\
46 \\
33 \\
48 \\
44 \\
9 \\
15 \\
11 \\
35 \\
29 \\
21 \\
\text { ND }\end{array}$ & $\begin{array}{r}0.6 \\
\text { ND } \\
0.9 \\
0.6 \\
<0.2 \\
0.7 \\
1.1 \\
0.4 \\
0.9 \\
<0.2 \\
0.9 \\
0.3 \\
0.7 \\
0.6 \\
<0.2 \\
<0.2 \\
<0.2 \\
0.6 \\
0.7 \\
0.3 \\
\text { ND }\end{array}$ & $\begin{array}{r}100 \\
\text { ND } \\
110 \\
86 \\
90 \\
67 \\
140 \\
110 \\
74 \\
40 \\
69 \\
71 \\
55 \\
71 \\
17 \\
34 \\
58 \\
50 \\
42 \\
46 \\
\text { ND }\end{array}$ & $\begin{array}{l}430 \\
\text { ND } \\
300 \\
140 \\
190 \\
130 \\
180 \\
230 \\
190 \\
270 \\
160 \\
160 \\
250 \\
240 \\
380 \\
310 \\
170 \\
150 \\
370 \\
260 \\
\text { ND }\end{array}$ & $\begin{array}{l}<5 \\
\text { ND } \\
<5 \\
<5 \\
<5 \\
<5 \\
<5 \\
17 \\
<5 \\
<5 \\
<5 \\
<5 \\
19 \\
23 \\
42 \\
27 \\
16 \\
<5 \\
18 \\
12 \\
\text { ND }\end{array}$ & $\begin{array}{l}43.9 \\
\text { ND } \\
34.9 \\
24.8 \\
35.6 \\
31.1 \\
37.8 \\
34.6 \\
33.8 \\
21.6 \\
35.1 \\
32.8 \\
33.4 \\
31.7 \\
18.9 \\
25.5 \\
18.8 \\
32.7 \\
32.2 \\
27.9 \\
\text { ND }\end{array}$ & $\begin{array}{r}650 \\
\text { ND } \\
370 \\
360 \\
300 \\
150 \\
710 \\
710 \\
1900 \\
89 \\
860 \\
300 \\
520 \\
460 \\
<50 \\
<50 \\
68 \\
300 \\
240 \\
100 \\
\text { ND }\end{array}$ & $\begin{array}{l}<1 \\
\text { ND } \\
<1 \\
<1 \\
<1 \\
<1 \\
<1 \\
<1 \\
<1 \\
<1 \\
<1 \\
<1 \\
<1 \\
<1 \\
<1 \\
<1 \\
<1 \\
<1 \\
<1 \\
<1 \\
\text { ND }\end{array}$ & $\begin{array}{l}<0.02 \\
\mathrm{ND} \\
<0.02 \\
<0.02 \\
<0.01 \\
<0.02 \\
<0.02 \\
<0.02 \\
<0.02 \\
<0.02 \\
<0.02 \\
<0.02 \\
<0.02 \\
<0.02 \\
<0.02 \\
<0.02 \\
<0.01 \\
<0.02 \\
<0.02 \\
<0.01 \\
\mathrm{ND}\end{array}$ & $\begin{array}{c}<4 \\
\text { ND } \\
<4 \\
<4 \\
<4 \\
<4 \\
<4 \\
<4 \\
<4 \\
<4 \\
<4 \\
<4 \\
<4 \\
<4 \\
<4 \\
<4 \\
<4 \\
<4 \\
<4 \\
<4 \\
\text { ND }\end{array}$ & $\begin{array}{r}<1 \\
\text { ND } \\
4 \\
2 \\
5 \\
3 \\
<1 \\
4 \\
<1 \\
9 \\
3 \\
4 \\
<1 \\
3 \\
<1 \\
4 \\
6 \\
4 \\
5 \\
3 \\
\text { ND }\end{array}$ & $\begin{array}{l}<5 \\
\text { ND } \\
<5 \\
<5 \\
<5 \\
<5 \\
<5 \\
<5 \\
<5 \\
<5 \\
<5 \\
<5 \\
<5 \\
<5 \\
<5 \\
<5 \\
<5 \\
<5 \\
<5 \\
<5 \\
<5 \\
<5 \\
<5 \\
\text { ND }\end{array}$ & $\begin{array}{r}12 \\
\text { ND } \\
9.2 \\
5.4 \\
9.3 \\
6.9 \\
4.2 \\
<0.5 \\
3.7 \\
8.1 \\
2.7 \\
<0.5 \\
4.3 \\
4.8 \\
<0.5 \\
<0.5 \\
1.7 \\
3.9 \\
9.4 \\
2.7 \\
\text { ND }\end{array}$ \\
\hline $\begin{array}{c}158-957 \mathrm{E}- \\
1 \mathrm{R}-1,23-26 \\
9 \mathrm{R}-1,16-19 \\
12 \mathrm{R}-1,16-20 \\
15 \mathrm{R}-1,27-30\end{array}$ & $\begin{array}{l}4 \\
4 \\
3 \\
6\end{array}$ & $\begin{array}{r}31.70 \\
78.16 \\
91.95 \\
106.77\end{array}$ & & $\begin{array}{r}55 \\
182 \\
87 \\
58\end{array}$ & $\begin{array}{r}<5 \\
<5 \\
7 \\
<5\end{array}$ & $\begin{array}{l}14 \\
32 \\
26 \\
43\end{array}$ & $\begin{array}{r}0.2 \\
<0.2 \\
0.8 \\
0.5\end{array}$ & $\begin{array}{r}31 \\
160 \\
130 \\
62\end{array}$ & $\begin{array}{l}250 \\
360 \\
330 \\
420\end{array}$ & $\begin{array}{l}15 \\
<5 \\
86 \\
29\end{array}$ & $\begin{array}{l}21 \\
33.9 \\
45.8 \\
30.9\end{array}$ & $\begin{array}{r}69 \\
180 \\
210 \\
140\end{array}$ & $\begin{array}{l}<1 \\
<1 \\
<1 \\
<1\end{array}$ & $\begin{array}{l}<0.01 \\
<0.02 \\
<0.01 \\
<0.01\end{array}$ & $\begin{array}{l}<4 \\
<4 \\
<4 \\
<4\end{array}$ & $\begin{array}{l}3 \\
6 \\
6 \\
2\end{array}$ & $\begin{array}{l}<5 \\
<5 \\
<5 \\
<5\end{array}$ & $\begin{array}{r}1.5 \\
<0.7 \\
4.3 \\
3.7\end{array}$ \\
\hline $\begin{array}{l}158-957 \mathrm{~F}- \\
1 \mathrm{~N}-1,32-35 \\
2 \mathrm{~N}-1,11-19\end{array}$ & $\begin{array}{l}8 \\
3\end{array}$ & $\begin{array}{l}1.41 \\
5.60\end{array}$ & & $\begin{array}{l}269 \\
329\end{array}$ & $\begin{array}{l}<5 \\
<5\end{array}$ & $\begin{array}{l}49 \\
57\end{array}$ & $\begin{array}{l}1.0 \\
1.1\end{array}$ & $\begin{array}{l}82 \\
97\end{array}$ & $\begin{array}{l}390 \\
240\end{array}$ & $\begin{array}{l}42 \\
13\end{array}$ & $\begin{array}{l}30.3 \\
38.7\end{array}$ & $\begin{array}{l}280 \\
300\end{array}$ & $\begin{array}{l}<1 \\
<1\end{array}$ & $\begin{array}{l}<0.02 \\
<0.01\end{array}$ & $\begin{array}{l}<4 \\
<4\end{array}$ & $\begin{array}{r}4 \\
<1\end{array}$ & $\begin{array}{l}<5 \\
<5\end{array}$ & $\begin{array}{l}4.4 \\
15\end{array}$ \\
\hline $\begin{array}{l}158-957 \mathrm{H}- \\
1 \mathrm{~N}-1,60-73 \\
5 \mathrm{~N}-1,61-64 \\
6 \mathrm{~N}-1,14-19\end{array}$ & $\begin{array}{c}13 \\
6 \\
3\end{array}$ & $\begin{array}{r}9.30 \\
27.30 \\
31.34\end{array}$ & & $\begin{array}{l}809 \\
128 \\
350\end{array}$ & $\begin{array}{r}7 \\
<5 \\
<5\end{array}$ & $\begin{array}{l}27 \\
20 \\
32\end{array}$ & $\begin{array}{l}0.6 \\
1.2 \\
0.8\end{array}$ & $\begin{array}{r}73 \\
110 \\
72\end{array}$ & $\begin{array}{l}200 \\
390 \\
260\end{array}$ & $\begin{array}{l}16 \\
43 \\
15\end{array}$ & $\begin{array}{l}33 \\
36.1 \\
30.5\end{array}$ & $\begin{array}{r}170 \\
230 \\
75\end{array}$ & $\begin{array}{l}<1 \\
<1 \\
<1\end{array}$ & $\begin{array}{l}<0.01 \\
<0.01 \\
<0.01\end{array}$ & $\begin{array}{l}<4 \\
<4 \\
<4\end{array}$ & $\begin{array}{l}4 \\
5 \\
7\end{array}$ & $\begin{array}{l}<5 \\
<5 \\
<5\end{array}$ & $\begin{array}{l}4.3 \\
3.5 \\
4.9\end{array}$ \\
\hline $\begin{array}{l}\text { 158-957I- } \\
1 \mathrm{~N}-1,33-41\end{array}$ & 6 & 9.33 & & 431 & $<5$ & 52 & 4.8 & 220 & 33 & $<54$ & 4.5 & 35000 & $<1$ & $<0.02$ & $<4$ & 7 & $<5$ & $<0.8$ \\
\hline $\begin{array}{l}158-957 \mathrm{~K}- \\
1 \mathrm{X}-1,24-29 \\
1 \mathrm{~N}-1,37-41 \\
3 \mathrm{X}-1,36-38\end{array}$ & $\begin{array}{l}4 \\
9 \\
7\end{array}$ & $\begin{array}{r}0.21 \\
10.37 \\
14.82\end{array}$ & & $\begin{array}{r}409 \\
830 \\
1500\end{array}$ & $\begin{array}{l}<5 \\
<5 \\
<5\end{array}$ & $\begin{array}{l}45 \\
55 \\
78\end{array}$ & $\begin{array}{l}3.4 \\
3.0 \\
7.6\end{array}$ & $\begin{array}{l}160 \\
120 \\
110\end{array}$ & $\begin{array}{l}15 \\
56 \\
61\end{array}$ & $\begin{array}{l}<54 \\
<5 \\
<5\end{array}$ & $\begin{array}{r}0.9 \\
46.7 \\
43.6\end{array}$ & $\begin{array}{r}30000 \\
8400 \\
39000\end{array}$ & $\begin{array}{l}<1 \\
<1 \\
<1\end{array}$ & $\begin{array}{l}<0.02 \\
<0.01 \\
<0.02\end{array}$ & $\begin{array}{l}<4 \\
<4 \\
<4\end{array}$ & $\begin{array}{l}8 \\
4 \\
6\end{array}$ & $\begin{array}{l}<5 \\
<5 \\
<5\end{array}$ & $\begin{array}{l}<0.9 \\
11 \\
9.8\end{array}$ \\
\hline $\begin{array}{l}\text { 158-957M- } \\
\text { 1R-1, 16-47 } \\
\text { 1R-1, 16-47 } \\
\text { 1R-1, 16-47 } \\
\text { 1R-2, 8-12 } \\
\text { 2R-1, 2-5 } \\
\text { 3R-1, 57-60 } \\
\text { 3R-1, 123-125 } \\
\text { 3R-2, 1-4 } \\
\text { 5R-1, 90-92 } \\
\text { 7R-1, 0-4 } \\
\text { 8R-1, 0-4 }\end{array}$ & $\begin{array}{c}-63 \\
420-63 \\
+420 \\
3 \\
1 \\
14 \\
28 \\
1 \\
15 \\
1 \\
1\end{array}$ & $\begin{array}{r}0.00 \\
0.00 \\
0.00 \\
0.63 \\
9.30 \\
14.87 \\
15.49 \\
15.80 \\
25.18 \\
34.30 \\
38.30\end{array}$ & & $\begin{array}{l}183 \\
\text { ND } \\
\text { ND } \\
135 \\
1770 \\
1050 \\
367 \\
504 \\
263 \\
199 \\
893\end{array}$ & $\begin{array}{r}<5 \\
\text { ND } \\
\text { ND } \\
<5 \\
19 \\
<5 \\
<5 \\
<5 \\
11 \\
<5 \\
<5\end{array}$ & $\begin{array}{r}140 \\
\text { ND } \\
\text { ND } \\
18 \\
140 \\
65 \\
49 \\
35 \\
35 \\
8 \\
65\end{array}$ & $\begin{array}{r}6.9 \\
\text { ND } \\
\text { ND } \\
1.0 \\
6.5 \\
3.5 \\
1.7 \\
1.3 \\
1.3 \\
<0.2 \\
3.7\end{array}$ & $\begin{array}{r}82 \\
\text { ND } \\
\text { ND } \\
17 \\
86 \\
150 \\
130 \\
76 \\
65 \\
9 \\
180\end{array}$ & $\begin{array}{r}14 \\
\text { ND } \\
\text { ND } \\
<5 \\
180 \\
83 \\
220 \\
410 \\
270 \\
100 \\
230\end{array}$ & $\begin{array}{l}<55 \\
\text { ND } \\
\text { ND } \\
<5 \\
<5 \\
<5 \\
<5 \\
15 \\
<5 \\
<5 \\
<5\end{array}$ & \begin{tabular}{l}
\multicolumn{1}{c}{4.0} \\
ND \\
ND \\
12.4 \\
48.9 \\
43.3 \\
34.9 \\
28.8 \\
25.1 \\
13.0 \\
38.2
\end{tabular} & $\begin{array}{r}2600 \\
\text { ND } \\
\text { ND } \\
230 \\
640 \\
43000 \\
8600 \\
340 \\
490 \\
670 \\
7100\end{array}$ & $\begin{array}{l}<1 \\
\text { ND } \\
\text { ND } \\
<1 \\
<1 \\
<1 \\
<1 \\
<1 \\
<1 \\
<1 \\
<1\end{array}$ & $\begin{array}{l}<0.02 \\
\text { ND } \\
\text { ND } \\
<0.01 \\
<0.03 \\
<0.01 \\
<0.01 \\
<0.01 \\
<0.02 \\
<0.02 \\
<0.01\end{array}$ & $\begin{array}{l}<4 \\
\text { ND } \\
\mathrm{ND} \\
<4 \\
<4 \\
<4 \\
<4 \\
<4 \\
<4 \\
<4 \\
<4\end{array}$ & $\begin{array}{r}86 \\
\text { ND } \\
\text { ND } \\
9 \\
<1 \\
<2 \\
4 \\
3 \\
4 \\
9 \\
3\end{array}$ & $\begin{array}{l}<5 \\
\text { ND } \\
\text { ND } \\
<5 \\
<5 \\
<5 \\
<5 \\
<5 \\
<5 \\
<5 \\
<5 \\
<5\end{array}$ & $\begin{array}{c}8.5 \\
\text { ND } \\
\text { ND } \\
2.3 \\
16 \\
7.2 \\
10 \\
12 \\
12 \\
3.6 \\
30\end{array}$ \\
\hline $\begin{array}{l}158-957 \mathrm{O}- \\
2 \mathrm{R}-1,22-24 \\
4 \mathrm{R}-1,45-50\end{array}$ & $\begin{array}{l}5 \\
9\end{array}$ & $\begin{array}{r}8.10 \\
16.35\end{array}$ & & $\begin{array}{l}150 \\
274\end{array}$ & $\begin{array}{l}<5 \\
<5\end{array}$ & $\begin{array}{l}34 \\
61\end{array}$ & $\begin{array}{l}0.8 \\
1.6\end{array}$ & $\begin{array}{l}110 \\
130\end{array}$ & $\begin{array}{l}220 \\
270\end{array}$ & $\begin{array}{l}42 \\
45\end{array}$ & $\begin{array}{l}32.1 \\
43.7\end{array}$ & $\begin{array}{l}340 \\
390\end{array}$ & $\begin{array}{l}<1 \\
<1\end{array}$ & $\begin{array}{l}<0.01 \\
<0.01\end{array}$ & $\begin{array}{l}<4 \\
<4\end{array}$ & $\begin{array}{r}4 \\
<1\end{array}$ & $\begin{array}{l}<5 \\
<5\end{array}$ & $\begin{array}{l}3.6 \\
8.5\end{array}$ \\
\hline $\begin{array}{l}\text { 158-957P- } \\
\text { 1R-1, 44-46 } \\
5 \mathrm{R}-1,9-13 \\
5 \mathrm{R}-1,9-13 \\
\text { 9R-1, 11-13 } \\
\text { 10R-1, 1-3 } \\
\text { 12R-1, 15-100 } \\
\text { 12R-1, 15-100 } \\
\text { 12R-1, 15-100 } \\
\text { 12R-2, 25-28 } \\
\text { 12R-4, 53-57 } \\
\text { 13W-1, 50-58 } \\
13 \mathrm{~W}-1+2,0-150\end{array}$ & $\begin{array}{c}8 \\
2 \\
2 \\
2 \\
1 \\
-63 \\
420-63 \\
+420 \\
6 \\
5 \\
- \\
-\end{array}$ & $\begin{array}{r}0.43 \\
21.57 \\
21.57 \\
40.19 \\
45.10 \\
54.40 \\
54.40 \\
54.40 \\
55.89 \\
57.61 \\
15.00 \\
15.00\end{array}$ & & $\begin{array}{c}130 \\
213 \\
\text { ND } \\
299 \\
193 \\
739 \\
\text { ND } \\
\text { ND } \\
51 \\
241 \\
523 \\
720\end{array}$ & $\begin{array}{r}<5 \\
<5 \\
\mathrm{ND} \\
<5 \\
<5 \\
<5 \\
\mathrm{ND} \\
\mathrm{ND} \\
<5 \\
<5 \\
<5 \\
9\end{array}$ & $\begin{array}{r}30 \\
70 \\
\text { ND } \\
46 \\
34 \\
74 \\
\text { ND } \\
\text { ND } \\
11 \\
51 \\
63 \\
69\end{array}$ & $\begin{array}{r}0.6 \\
0.8 \\
\text { ND } \\
1.3 \\
0.5 \\
2.5 \\
\text { ND } \\
\text { ND } \\
<0.2 \\
1.0 \\
1.3 \\
2.1\end{array}$ & $\begin{array}{r}57 \\
99 \\
\text { ND } \\
99 \\
87 \\
130 \\
\text { ND } \\
\text { ND } \\
33 \\
210 \\
130 \\
88\end{array}$ & $\begin{array}{l}220 \\
350 \\
\text { ND } \\
310 \\
400 \\
230 \\
\text { ND } \\
\text { ND } \\
550 \\
570 \\
380 \\
410\end{array}$ & $\begin{array}{c}30 \\
<54 \\
\text { ND } \\
14 \\
41 \\
34 \\
\text { ND } \\
\text { ND } \\
33 \\
43 \\
50 \\
41\end{array}$ & $\begin{array}{l}25.5 \\
2.6 \\
\text { ND } \\
37.1 \\
43 \\
37.6 \\
\text { ND } \\
\text { ND } \\
24 \\
43.6 \\
42.5 \\
46.8\end{array}$ & $\begin{array}{r}91 \\
750 \\
\text { ND } \\
370 \\
340 \\
5500 \\
\text { ND } \\
\text { ND } \\
160 \\
190 \\
1600 \\
3000\end{array}$ & $\begin{array}{c}<1 \\
<1 \\
\text { ND } \\
<1 \\
<1 \\
<1 \\
\text { ND } \\
\text { ND } \\
1 \\
<1 \\
<1 \\
6\end{array}$ & $\begin{array}{c}<0.01 \\
<0.01 \\
\text { ND } \\
<0.01 \\
<0.02 \\
<0.02 \\
\text { ND } \\
\text { ND } \\
<0.01 \\
<0.02 \\
<0.01 \\
<0.03\end{array}$ & $\begin{array}{l}<4 \\
<4 \\
N D \\
<4 \\
<4 \\
<4 \\
\text { ND } \\
\text { ND } \\
<4 \\
<4 \\
<4 \\
<4\end{array}$ & $\begin{array}{r}5 \\
3 \\
\mathrm{ND} \\
3 \\
<1 \\
20 \\
\mathrm{ND} \\
\mathrm{ND} \\
2 \\
<1 \\
15 \\
22\end{array}$ & $\begin{array}{l}<5 \\
<5 \\
\text { ND } \\
<5 \\
<5 \\
<5 \\
\text { ND } \\
\text { ND } \\
<5 \\
<5 \\
<5 \\
<5\end{array}$ & $\begin{array}{c}2.5 \\
2.8 \\
\text { ND } \\
13 \\
18 \\
4.5 \\
\text { ND } \\
\text { ND } \\
4.1 \\
22 \\
3.7 \\
5.7\end{array}$ \\
\hline $\begin{array}{l}\text { 158-957Q- } \\
\text { 1R-1, 13-18 } \\
\text { 1R-3, 70-100 } \\
\text { 1R-3, 119-121 } \\
\text { 2R-1, 0-4 }\end{array}$ & $\frac{-}{1}$ & $\begin{array}{l}0.13 \\
3.75 \\
4.10 \\
9.50\end{array}$ & & $\begin{array}{r}1940 \\
1110 \\
1400 \\
885\end{array}$ & $\begin{array}{r}8 \\
<5 \\
<5 \\
27\end{array}$ & $\begin{array}{l}56 \\
45 \\
13 \\
10\end{array}$ & $\begin{array}{l}4.2 \\
1.9 \\
1.3 \\
1.7\end{array}$ & $\begin{array}{r}37 \\
75 \\
7 \\
6\end{array}$ & $\begin{array}{r}25 \\
130 \\
10 \\
<5\end{array}$ & $\begin{array}{l}<51 \\
12 \\
<51 \\
<5\end{array}$ & $\begin{array}{c}8.0 \\
36.8 \\
0.8 \\
4.21\end{array}$ & $\begin{array}{l}9800 \\
4400 \\
4600 \\
1200\end{array}$ & $\begin{array}{l}<1 \\
<1 \\
<1 \\
<1\end{array}$ & $\begin{array}{l}<0.03 \\
<0.01 \\
<0.02 \\
<0.01\end{array}$ & $\begin{array}{l}<4 \\
<4 \\
<4 \\
<4\end{array}$ & $\begin{array}{l}26 \\
15 \\
40 \\
10\end{array}$ & $\begin{array}{l}<5 \\
<5 \\
<5 \\
<5\end{array}$ & $\begin{array}{l}5.2 \\
5.4 \\
6.4 \\
2.3\end{array}$ \\
\hline
\end{tabular}


Table 5. Rare earth element composition of samples from Site 957.

\begin{tabular}{|c|c|c|c|c|c|c|c|c|c|c|c|c|c|c|c|c|c|c|}
\hline $\begin{array}{l}\text { Core, section, } \\
\text { interval }(\mathrm{cm})\end{array}$ & Piece & $\begin{array}{l}\text { Depth } \\
\text { (mbsf) }\end{array}$ & $\begin{array}{l}\text { Element: } \\
\text { Method: } \\
\text { Detection } \\
\text { limit: }\end{array}$ & $\begin{array}{c}\mathrm{La} \\
\text { ICP-MS }\end{array}$ & $\begin{array}{c}\mathrm{Ce} \\
\text { ICP-MS }\end{array}$ & $\begin{array}{c}\text { Pr } \\
\text { ICP-MS }\end{array}$ & $\begin{array}{c}\mathrm{Nd} \\
\text { ICP-MS }\end{array}$ & $\begin{array}{c}\mathrm{Sm} \\
\text { ICP-MS }\end{array}$ & $\begin{array}{c}\text { Eu } \\
\text { ICP-MS }\end{array}$ & $\begin{array}{c}\mathrm{Gd} \\
\text { ICP-MS }\end{array}$ & $\begin{array}{c}\mathrm{Tb} \\
\text { ICP-MS }\end{array}$ & $\begin{array}{c}\text { Dy } \\
\text { ICP-MS }\end{array}$ & $\begin{array}{c}\text { Ho } \\
\text { ICP-MS }\end{array}$ & $\begin{array}{c}\text { Er } \\
\text { ICP-MS }\end{array}$ & $\begin{array}{c}\mathrm{Tm} \\
\text { ICP-MS }\end{array}$ & $\begin{array}{c}\mathrm{Yb} \\
\text { ICP-MS } \\
0.05 \mathrm{ppm}\end{array}$ & $\begin{array}{c}\mathrm{Lu} \\
\text { ICP-MS }\end{array}$ & $\begin{array}{c}\mathrm{Y} \\
\text { ICP-MS }\end{array}$ \\
\hline $\begin{array}{c}\text { 158-957A- } \\
3 \mathrm{X}-1,4-8\end{array}$ & 1 & 10.00 & & ND & ND & ND & ND & ND & ND & ND & ND & ND & ND & ND & ND & ND & ND & ND \\
\hline $\begin{array}{c}\text { 158-957B- } \\
1 \mathrm{R}-1,3-95 \\
1 \mathrm{R}-1,3-95 \\
1 \mathrm{R}-1,3-95 \\
1 \mathrm{R}-1,3-95 \\
4 \mathrm{R}-1,0-10\end{array}$ & $\begin{array}{c}-63 \\
420-63 \\
+420 \\
+420 \\
1\end{array}$ & $\begin{array}{r}0.00 \\
0.00 \\
0.00 \\
0.00 \\
19.90\end{array}$ & & $\begin{array}{l}1.8 \\
1.0 \\
0.7 \\
0.7 \\
\text { ND }\end{array}$ & $\begin{array}{l}4.7 \\
2.6 \\
1.6 \\
1.6 \\
\text { ND }\end{array}$ & $\begin{array}{l}0.86 \\
0.47 \\
0.28 \\
0.28 \\
\mathrm{ND}\end{array}$ & $\begin{array}{l}4.1 \\
2.3 \\
1.4 \\
1.5 \\
\text { ND }\end{array}$ & $\begin{array}{l}1.30 \\
0.81 \\
0.43 \\
0.43 \\
\mathrm{ND}\end{array}$ & $\begin{array}{l}4.0 \\
2.2 \\
1.4 \\
1.2 \\
\text { ND }\end{array}$ & $\begin{array}{l}1.4 \\
0.81 \\
0.42 \\
0.40 \\
\mathrm{ND}\end{array}$ & $\begin{array}{l}0.21 \\
0.11 \\
0.07 \\
0.06 \\
\text { ND }\end{array}$ & $\begin{array}{l}1.0 \\
0.57 \\
0.30 \\
0.30 \\
\text { ND }\end{array}$ & $\begin{array}{l}0.18 \\
0.09 \\
0.05 \\
0.05 \\
\text { ND }\end{array}$ & $\begin{array}{l}0.42 \\
0.23 \\
0.14 \\
0.13 \\
\mathrm{ND}\end{array}$ & $\begin{array}{c}0.07 \\
0.04 \\
<0.02 \\
0.02 \\
\text { ND }\end{array}$ & $\begin{array}{l}0.39 \\
0.21 \\
0.13 \\
0.12 \\
\mathrm{ND}\end{array}$ & $\begin{array}{c}0.06 \\
0.03 \\
<0.02 \\
<0.02 \\
\text { ND }\end{array}$ & $\begin{array}{l}4.2 \\
2.3 \\
1.4 \\
1.4 \\
\text { ND }\end{array}$ \\
\hline $\begin{array}{l}158-957 \mathrm{C}- \\
5 \mathrm{~N}-1,15-48 \\
5 \mathrm{~N}-1,15-48 \\
7 \mathrm{~N}-1,52-56 \\
7 \mathrm{~N}-2,63-69 \\
10 \mathrm{~N}-1,21-22 \\
11 \mathrm{~N}-1,58-61 \\
11 \mathrm{~N}-3,90-94 \\
12 \mathrm{~N}-1,53-59 \\
12 \mathrm{~N}-2,68-75 \\
12 \mathrm{~N}-3,86-100 \\
13 \mathrm{~N}-1,12-20 \\
14 \mathrm{~N}-1,33-41 \\
14 \mathrm{~N}-2,76.5-88 \\
15 \mathrm{~N}-1,36-40 \\
15 \mathrm{~N}-2,12-15 \\
15 \mathrm{~N}-3,132-150 \\
15 \mathrm{~N}-4,8-10 \\
16 \mathrm{~N}-1,118-125 \\
16 \mathrm{~N}-2,14-123 \\
16 \mathrm{~N}-2,94-95 \\
16 \mathrm{~N}-2,94-95\end{array}$ & $\begin{array}{c}3,5,6 \\
3,5,6 \\
6 \mathrm{~F} \\
1 \mathrm{E} \\
4 \\
3 \mathrm{D} \\
8 \mathrm{C} \\
6 \mathrm{~A} \\
9 \\
9 \\
4 \\
5 \\
6 \\
5 \\
1 \mathrm{~B} \\
11 \\
1 \\
15 \\
2 \\
9 \mathrm{~B} \\
9 \mathrm{~B}\end{array}$ & $\begin{array}{l}15.25 \\
15.25 \\
20.04 \\
21.45 \\
28.86 \\
31.18 \\
34.50 \\
35.45 \\
36.16 \\
36.94 \\
37.31 \\
40.53 \\
41.67 \\
42.50 \\
43.28 \\
45.54 \\
45.70 \\
47.39 \\
47.75 \\
48.56 \\
48.56\end{array}$ & & $\begin{array}{r}<0.1 \\
\text { ND } \\
0.2 \\
0.2 \\
0.1 \\
0.1 \\
<0.1 \\
0.1 \\
0.1 \\
0.2 \\
<0.1 \\
<0.1 \\
0.1 \\
0.2 \\
0.4 \\
0.2 \\
0.3 \\
0.1 \\
<0.1 \\
0.3 \\
0.3\end{array}$ & $\begin{array}{l}0.2 \\
\text { ND } \\
0.2 \\
0.6 \\
0.3 \\
0.3 \\
0.1 \\
0.3 \\
0.3 \\
0.4 \\
0.1 \\
0.3 \\
0.4 \\
0.5 \\
1.2 \\
0.6 \\
0.9 \\
0.4 \\
0.3 \\
1.1 \\
1.1\end{array}$ & $\begin{array}{l}0.04 \\
\text { ND } \\
0.03 \\
0.10 \\
0.04 \\
0.05 \\
0.02 \\
0.04 \\
0.05 \\
0.07 \\
0.02 \\
0.05 \\
0.06 \\
0.08 \\
0.21 \\
0.10 \\
0.14 \\
0.09 \\
0.05 \\
0.21 \\
0.20\end{array}$ & $\begin{array}{r}0.2 \\
\text { ND } \\
0.2 \\
0.5 \\
0.2 \\
0.2 \\
0.1 \\
0.2 \\
0.2 \\
0.4 \\
<0.1 \\
0.2 \\
0.3 \\
0.4 \\
1.1 \\
0.5 \\
0.7 \\
0.5 \\
0.2 \\
1.1 \\
1.1\end{array}$ & $\begin{array}{c}0.08 \\
\text { ND } \\
0.04 \\
0.10 \\
0.04 \\
0.05 \\
0.02 \\
0.05 \\
0.05 \\
0.10 \\
<0.02 \\
0.05 \\
0.08 \\
0.11 \\
0.29 \\
0.15 \\
0.19 \\
0.12 \\
0.07 \\
0.30 \\
0.29\end{array}$ & $\begin{array}{l}0.10 \\
\text { ND } \\
0.08 \\
0.26 \\
0.09 \\
0.11 \\
0.04 \\
0.07 \\
0.12 \\
0.14 \\
0.05 \\
0.09 \\
0.10 \\
0.13 \\
0.24 \\
0.14 \\
0.30 \\
0.19 \\
0.06 \\
0.20 \\
0.19\end{array}$ & $\begin{array}{c}0.06 \\
\text { ND } \\
0.04 \\
0.07 \\
0.04 \\
0.06 \\
0.03 \\
0.06 \\
0.04 \\
0.10 \\
<0.02 \\
0.04 \\
0.06 \\
0.10 \\
0.27 \\
0.12 \\
0.18 \\
0.08 \\
0.07 \\
0.17 \\
0.17\end{array}$ & $\begin{array}{c}<0.02 \\
N D \\
<<0.02 \\
<0.02 \\
<0.02 \\
<0.02 \\
<0.02 \\
<0.02 \\
<0.02 \\
<0.02 \\
<0.02 \\
<0.02 \\
<0.02 \\
<0.02 \\
0.04 \\
<0.02 \\
0.03 \\
<0.02 \\
<<.02 \\
<0.02 \\
<0.02\end{array}$ & $\begin{array}{c}0.06 \\
\mathrm{ND} \\
0.04 \\
0.06 \\
0.04 \\
0.05 \\
0.02 \\
0.06 \\
0.02 \\
0.11 \\
<0.02 \\
0.03 \\
0.04 \\
0.06 \\
0.21 \\
0.06 \\
0.13 \\
0.05 \\
0.07 \\
0.07 \\
0.06\end{array}$ & $\begin{array}{c}<0.02 \\
N D \\
<0.02 \\
<0.02 \\
<0.02 \\
<0.02 \\
<0.02 \\
<0.02 \\
<0.02 \\
<0.02 \\
<0.02 \\
<0.02 \\
<0.02 \\
<0.02 \\
0.04 \\
<0.02 \\
0.03 \\
00.02 \\
<0.02 \\
<0.02 \\
<0.02\end{array}$ & $\begin{array}{r}<0.02 \\
\text { ND } \\
<0.02 \\
0.02 \\
<0.02 \\
<0.02 \\
<0.02 \\
0.04 \\
<0.02 \\
0.05 \\
<0.02 \\
<0.02 \\
<0.02 \\
0.03 \\
0.11 \\
<0.02 \\
0.06 \\
<0.02 \\
0.05 \\
<0.02 \\
<0.02\end{array}$ & $\begin{array}{l}<0.02 \\
\mathrm{ND} \\
<<0.02 \\
<0.02 \\
<0.02 \\
<0.02 \\
<0.02 \\
<0.02 \\
<0.02 \\
<0.02 \\
<0.02 \\
<0.02 \\
<0.02 \\
<0.02 \\
<0.02 \\
<0.02 \\
<0.02 \\
<0.02 \\
<0.02 \\
<0.02 \\
<0.02\end{array}$ & $\begin{array}{c}<0.05 \\
\mathrm{ND} \\
<0.05 \\
<0.05 \\
<0.05 \\
<0.05 \\
<0.05 \\
0.05 \\
<0.05 \\
0.05 \\
<0.05 \\
<0.05 \\
<0.05 \\
<0.05 \\
0.11 \\
<0.05 \\
0.07 \\
<0.05 \\
0.06 \\
<0.05 \\
<0.05\end{array}$ & $\begin{array}{l}<0.02 \\
\text { ND } \\
<<.02 \\
<0.02 \\
<0.02 \\
<0.02 \\
<0.02 \\
<0.02 \\
<0.02 \\
<0.02 \\
<0.02 \\
<0.02 \\
<0.02 \\
<0.02 \\
<0.02 \\
<0.02 \\
<0.02 \\
<0.02 \\
<0.02 \\
<0.02 \\
<0.02\end{array}$ & $\begin{array}{l}0.18 \\
\text { ND } \\
0.13 \\
0.23 \\
0.19 \\
0.18 \\
0.08 \\
0.39 \\
0.12 \\
0.52 \\
0.06 \\
0.11 \\
0.14 \\
0.31 \\
1.0 \\
0.20 \\
0.72 \\
0.16 \\
0.38 \\
0.16 \\
0.19\end{array}$ \\
\hline $\begin{array}{l}\text { 158-957E- } \\
\text { 1R-1, 23-26 } \\
9 \mathrm{R}-1,16-19 \\
\text { 12R-1,16-20 } \\
\text { 15R-1, 27-30 }\end{array}$ & $\begin{array}{l}4 \\
4 \\
3 \\
6\end{array}$ & $\begin{array}{r}31.70 \\
78.16 \\
91.95 \\
106.77\end{array}$ & & $\begin{array}{r}0.2 \\
0.2 \\
<0.1 \\
<0.1\end{array}$ & $\begin{array}{l}0.9 \\
0.6 \\
0.2 \\
0.3\end{array}$ & $\begin{array}{l}0.16 \\
0.11 \\
0.03 \\
0.05\end{array}$ & $\begin{array}{l}0.9 \\
0.6 \\
0.2 \\
0.3\end{array}$ & $\begin{array}{l}0.22 \\
0.17 \\
0.06 \\
0.11\end{array}$ & $\begin{array}{l}0.13 \\
0.07 \\
0.03 \\
0.06\end{array}$ & $\begin{array}{l}0.12 \\
0.11 \\
0.06 \\
0.09\end{array}$ & $\begin{array}{l}<0.02 \\
<0.02 \\
<0.02 \\
<0.02\end{array}$ & $\begin{array}{l}0.04 \\
0.07 \\
0.06 \\
0.07\end{array}$ & $\begin{array}{l}<0.02 \\
<0.02 \\
<0.02 \\
<0.02\end{array}$ & $\begin{array}{r}<0.02 \\
0.03 \\
0.04 \\
0.04\end{array}$ & $\begin{array}{l}<0.02 \\
<0.02 \\
<0.02 \\
<0.02\end{array}$ & $\begin{array}{l}<0.05 \\
<0.05 \\
<0.05 \\
<0.05\end{array}$ & $\begin{array}{l}<0.02 \\
<0.02 \\
<0.02 \\
<0.02\end{array}$ & $\begin{array}{l}0.11 \\
0.28 \\
0.33 \\
0.36\end{array}$ \\
\hline $\begin{array}{l}158-957 \mathrm{~F}- \\
1 \mathrm{~N}-1,32-35 \\
2 \mathrm{~N}-1,11-19\end{array}$ & $\begin{array}{l}8 \\
3\end{array}$ & $\begin{array}{l}1.41 \\
5.60\end{array}$ & & $\begin{array}{l}<0.1 \\
<0.1\end{array}$ & $\begin{array}{l}0.1 \\
0.3\end{array}$ & $\begin{array}{r}<0.02 \\
0.06\end{array}$ & $\begin{array}{r}<0.1 \\
0.2\end{array}$ & $\begin{array}{l}0.02 \\
0.07\end{array}$ & $\begin{array}{l}0.04 \\
0.14\end{array}$ & $\begin{array}{r}<0.02 \\
0.06\end{array}$ & $\begin{array}{l}<0.02 \\
<0.02\end{array}$ & $\begin{array}{r}<0.02 \\
0.05\end{array}$ & $\begin{array}{l}<0.02 \\
<0.02\end{array}$ & $\begin{array}{r}<0.02 \\
0.02\end{array}$ & $\begin{array}{l}<0.02 \\
<0.02\end{array}$ & $\begin{array}{l}<0.05 \\
<0.05\end{array}$ & $\begin{array}{l}<0.02 \\
<0.02\end{array}$ & $\begin{array}{l}0.08 \\
0.18\end{array}$ \\
\hline $\begin{array}{l}158-957 \mathrm{H}- \\
1 \mathrm{~N}-1,60-73 \\
5 \mathrm{~N}-1,61-64 \\
6 \mathrm{~N}-1,14-19\end{array}$ & $\begin{array}{c}13 \\
6 \\
3\end{array}$ & $\begin{array}{r}9.30 \\
27.30 \\
31.34\end{array}$ & & $\begin{array}{l}0.1 \\
0.2 \\
0.6\end{array}$ & $\begin{array}{l}0.3 \\
0.6 \\
1.8\end{array}$ & $\begin{array}{l}0.04 \\
0.10 \\
0.28\end{array}$ & $\begin{array}{l}0.2 \\
0.5 \\
1.3\end{array}$ & $\begin{array}{l}0.03 \\
0.14 \\
0.28\end{array}$ & $\begin{array}{l}0.13 \\
0.12 \\
0.31\end{array}$ & $\begin{array}{l}0.02 \\
0.10 \\
0.23\end{array}$ & $\begin{array}{r}<0.02 \\
<0.02 \\
0.03\end{array}$ & $\begin{array}{r}<0.02 \\
0.09 \\
0.10\end{array}$ & $\begin{array}{l}<0.02 \\
<0.02 \\
<0.02\end{array}$ & $\begin{array}{r}<0.02 \\
0.04 \\
0.03\end{array}$ & $\begin{array}{l}<0.02 \\
<0.02 \\
<0.02\end{array}$ & $\begin{array}{l}<0.05 \\
<0.05 \\
<0.05\end{array}$ & $\begin{array}{l}<0.02 \\
<0.02 \\
<0.02\end{array}$ & $\begin{array}{l}0.07 \\
0.39 \\
0.29\end{array}$ \\
\hline $\begin{array}{l}158-957 \mathrm{I}- \\
1 \mathrm{~N}-1,33-41\end{array}$ & 6 & 9.33 & & $<0.1$ & $<0.1$ & $<0.02$ & $<0.1$ & $<0.02$ & $<0.02$ & $<0.02$ & $<0.02$ & $<0.02$ & $<0.02$ & $<0.02$ & $<0.02$ & $<0.05$ & $<0.02$ & 0.03 \\
\hline $\begin{array}{l}158-957 \mathrm{~K}- \\
1 \mathrm{X}-1,24-29 \\
1 \mathrm{~N}-1,37-41 \\
3 \mathrm{X}-1,36-38\end{array}$ & $\begin{array}{l}4 \\
9 \\
7\end{array}$ & $\begin{array}{r}0.21 \\
10.37 \\
14.82\end{array}$ & & $\begin{array}{l}<0.1 \\
<0.1 \\
<0.1\end{array}$ & $\begin{array}{r}<0.1 \\
0.1 \\
<0.1\end{array}$ & $\begin{array}{r}<0.02 \\
0.02 \\
0.02\end{array}$ & $\begin{array}{r}<0.1 \\
0.2 \\
0.2\end{array}$ & $\begin{array}{r}<0.02 \\
0.10 \\
0.15\end{array}$ & $\begin{array}{l}0.03 \\
0.27 \\
0.36\end{array}$ & $\begin{array}{r}<0.02 \\
0.12 \\
0.14\end{array}$ & $\begin{array}{r}<0.02 \\
<0.02 \\
0.02\end{array}$ & $\begin{array}{r}<0.02 \\
0.09 \\
0.10\end{array}$ & $\begin{array}{l}<0.02 \\
<0.02 \\
<0.02\end{array}$ & $\begin{array}{r}<0.02 \\
0.03 \\
0.03\end{array}$ & $\begin{array}{l}<0.02 \\
<0.02 \\
<0.02\end{array}$ & $\begin{array}{l}<0.05 \\
<0.05 \\
<0.05\end{array}$ & $\begin{array}{l}<0.02 \\
<0.02 \\
<0.02\end{array}$ & $\begin{array}{l}0.04 \\
0.31 \\
0.32\end{array}$ \\
\hline $\begin{array}{c}\text { 158-957M- } \\
1 \mathrm{R}-1,16-47 \\
1 \mathrm{R}-1,16-47 \\
\text { 1R-1, 16-47 } \\
\text { 1R-2, 8-12 } \\
\text { 2R-1, 2-5 } \\
\text { 3R-1, 57-60 }\end{array}$ & $\begin{array}{c}-63 \\
420-63 \\
+420 \\
3 \\
1 \\
14\end{array}$ & $\begin{array}{r}0.00 \\
0.00 \\
0.00 \\
0.63 \\
9.30 \\
14.87\end{array}$ & & $\begin{array}{r}1.3 \\
1.1 \\
1.1 \\
\text { ND } \\
\text { ND } \\
<0.1\end{array}$ & $\begin{array}{r}3.8 \\
3.5 \\
3.5 \\
\text { ND } \\
\text { ND } \\
<0.1\end{array}$ & $\begin{array}{c}0.64 \\
0.61 \\
0.62 \\
\text { ND } \\
\text { ND } \\
<0.02\end{array}$ & $\begin{array}{l}3.1 \\
2.9 \\
2.9 \\
\text { ND } \\
\text { ND } \\
0.1\end{array}$ & $\begin{array}{l}0.79 \\
0.80 \\
0.77 \\
\text { ND } \\
\text { ND } \\
0.07\end{array}$ & $\begin{array}{l}2.3 \\
2.4 \\
2.4 \\
\text { ND } \\
\text { ND } \\
0.13\end{array}$ & $\begin{array}{l}0.56 \\
0.56 \\
0.57 \\
\text { ND } \\
\text { ND } \\
0.07\end{array}$ & $\begin{array}{l}0.09 \\
0.09 \\
0.09 \\
\text { ND } \\
\text { ND } \\
<0.02\end{array}$ & $\begin{array}{l}0.48 \\
0.48 \\
0.45 \\
\text { ND } \\
\text { ND } \\
0.07\end{array}$ & $\begin{array}{c}0.09 \\
0.09 \\
0.09 \\
\text { ND } \\
\text { ND } \\
<0.02\end{array}$ & $\begin{array}{l}0.21 \\
0.22 \\
0.20 \\
\text { ND } \\
\text { ND } \\
0.03\end{array}$ & $\begin{array}{l}0.04 \\
0.04 \\
0.04 \\
\text { ND } \\
\text { ND } \\
<0.02\end{array}$ & $\begin{array}{l}0.25 \\
0.23 \\
0.23 \\
\text { ND } \\
\text { ND } \\
<0.05\end{array}$ & $\begin{array}{c}0.04 \\
0.03 \\
0.03 \\
\text { ND } \\
\text { ND } \\
<0.02\end{array}$ & $\begin{array}{l}1.8 \\
1.5 \\
1.6 \\
\text { ND } \\
\text { ND } \\
0.26\end{array}$ \\
\hline
\end{tabular}


Table 5 (continued).

\begin{tabular}{|c|c|c|c|c|c|c|c|c|c|c|c|c|c|c|c|c|c|c|}
\hline & & & $\begin{array}{l}\text { Element: } \\
\text { Method: }\end{array}$ & $\begin{array}{c}\mathrm{La} \\
\text { ICP-MS }\end{array}$ & $\begin{array}{c}\mathrm{Ce} \\
\text { ICP-MS }\end{array}$ & $\begin{array}{c}\text { Pr } \\
\text { ICP-MS }\end{array}$ & $\begin{array}{c}\mathrm{Nd} \\
\text { ICP-MS }\end{array}$ & $\begin{array}{c}\mathrm{Sm} \\
\text { ICP-MS }\end{array}$ & $\begin{array}{c}\text { Eu } \\
\text { ICP-MS }\end{array}$ & $\begin{array}{c}\mathrm{Gd} \\
\text { ICP-MS }\end{array}$ & $\begin{array}{c}\mathrm{Tb} \\
\text { ICP-MS }\end{array}$ & $\begin{array}{c}\text { Dy } \\
\text { ICP-MS }\end{array}$ & $\begin{array}{c}\text { Ho } \\
\text { ICP-MS }\end{array}$ & $\begin{array}{c}\text { Er } \\
\text { ICP-MS }\end{array}$ & $\begin{array}{c}\mathrm{Tm} \\
\text { ICP-MS }\end{array}$ & $\begin{array}{c}\mathrm{Yb} \\
\mathrm{ICP}-\mathrm{MS}\end{array}$ & $\begin{array}{c}\mathrm{Lu} \\
\text { ICP-MS }\end{array}$ & $\begin{array}{c}\mathrm{Y} \\
\text { ICP-MS }\end{array}$ \\
\hline $\begin{array}{l}\text { Core, section, } \\
\text { interval }(\mathrm{cm})\end{array}$ & Piece & $\begin{array}{l}\text { Depth } \\
(\mathrm{mbsf})\end{array}$ & $\begin{array}{l}\text { Detection } \\
\text { limit: }\end{array}$ & $0.1 \mathrm{ppm}$ & $0.1 \mathrm{ppm}$ & $0.02 \mathrm{ppm}$ & $0.1 \mathrm{ppm}$ & $0.02 \mathrm{ppm}$ & $0.02 \mathrm{ppm}$ & $0.02 \mathrm{ppm}$ & $0.02 \mathrm{ppm}$ & $0.02 \mathrm{ppm}$ & $0.02 \mathrm{ppm}$ & $0.02 \mathrm{ppm}$ & $0.02 \mathrm{ppm}$ & $0.05 \mathrm{ppm}$ & $0.02 \mathrm{ppm}$ & $0.02 \mathrm{ppm}$ \\
\hline $3 R-1,123-125$ & 28 & 15.49 & & $<0.1$ & 0.3 & 0.06 & 0.3 & 0.19 & 0.35 & 0.22 & 0.04 & 0.21 & 0.03 & 0.07 & $<0.02$ & 0.07 & $<0.02$ & 0.70 \\
\hline $3 R-2,1-4$ & 1 & 15.80 & & 0.3 & 1.4 & 0.27 & 1.6 & 0.47 & 0.45 & 0.32 & 0.04 & 0.17 & 0.02 & 0.04 & $<0.02$ & $<0.05$ & $<0.02$ & 0.43 \\
\hline $5 \mathrm{R}-1,90-92$ & 15 & 25.18 & & 0.1 & 0.4 & 0.06 & 0.3 & 0.10 & 0.15 & 0.09 & $<0.02$ & 0.11 & 0.02 & 0.06 & $<0.02$ & 0.07 & $<0.02$ & 0.48 \\
\hline $7 \mathrm{R}-1,0-4$ & 1 & 34.30 & & ND & ND & ND & ND & ND & ND & ND & ND & ND & ND & ND & ND & ND & ND & ND \\
\hline $8 \mathrm{R}-1,0-4$ & 1 & 38.30 & & $<0.1$ & 0.4 & 0.10 & 0.8 & 0.51 & 0.90 & 0.68 & 0.12 & 0.64 & 0.11 & 0.26 & 0.04 & 0.20 & 0.02 & 2.1 \\
\hline \multicolumn{19}{|l|}{ 158-957O- } \\
\hline $2 \mathrm{R}-1,22-24$ & 5 & 8.10 & & 0.1 & 0.4 & 0.06 & 0.4 & 0.09 & 0.10 & 0.08 & $<0.02$ & 0.06 & $<0.02$ & 0.02 & $<0.02$ & $<0.05$ & $<0.02$ & 0.23 \\
\hline $4 \mathrm{R}-1,45-50$ & 9 & 16.35 & & $<0.1$ & 0.2 & 0.04 & 0.2 & 0.04 & 0.06 & 0.05 & $<0.02$ & 0.04 & $<0.02$ & $<0.02$ & $<0.02$ & $<0.05$ & $<0.02$ & 0.15 \\
\hline \multicolumn{19}{|l|}{ 158-957P- } \\
\hline $1 \mathrm{R}-1,44-46$ & 8 & 0.43 & & 0.2 & 0.7 & 0.12 & 0.7 & 0.15 & 0.13 & 0.13 & $<0.02$ & 0.08 & $<0.02$ & 0.03 & $<0.02$ & $<0.05$ & $<0.02$ & 0.31 \\
\hline 5R-1, 9-13 & 2 & 21.57 & & 0.1 & 0.4 & 0.07 & 0.4 & 0.12 & 0.08 & 0.12 & $<0.02$ & 0.09 & $<0.02$ & 0.03 & $<0.02$ & $<0.05$ & $<0.02$ & 0.35 \\
\hline $5 \mathrm{R}-1,9-13$ & 2 & 21.57 & & 0.1 & 0.4 & 0.08 & 0.5 & 0.12 & 0.07 & 0.12 & $<0.02$ & 0.08 & $<0.02$ & 0.03 & $<0.02$ & $<0.05$ & $<0.02$ & 0.36 \\
\hline 9R-1, 11-13 & 2 & 40.19 & & $<0.1$ & 0.1 & 0.02 & 0.2 & 0.05 & 0.08 & 0.05 & $<0.02$ & 0.05 & $<0.02$ & 0.03 & $<0.02$ & $<0.05$ & $<0.02$ & 0.21 \\
\hline 10R-1, 1-3 & 1 & $\begin{array}{l}40.19 \\
45.10\end{array}$ & & $<0.1$ & $<0.1$ & $<0.02$ & $<0.1$ & 0.02 & $\begin{array}{l}0.02 \\
0.02\end{array}$ & 0.02 & $<0.02$ & 0.03 & $<0.02$ & $<0.02$ & $<0.02$ & $<0.05$ & $<0.02$ & 0.11 \\
\hline $12 \mathrm{R}-1,15-100$ & -63 & 54.40 & & $<0.1$ & 0.3 & 0.04 & 0.2 & 0.05 & 0.04 & 0.05 & $<0.02$ & 0.04 & $<0.02$ & 0.03 & $<0.02$ & $<0.05$ & $<0.02$ & 0.22 \\
\hline $12 \mathrm{R}-0,15-100$ & $420-63$ & 54.40 & & $<0.1$ & 0.3 & 0.04 & 0.2 & 0.06 & 0.0 & 0.06 & $<0.02$ & 0.05 & $<0.02$ & 0.03 & $<0.02$ & $<0.05$ & $<0.02$ & 0.27 \\
\hline $12 \mathrm{R}-1,15-100$ & +420 & 54.40 & & 0.1 & 0.4 & 0.06 & 0.3 & 0.09 & 0.07 & 0.10 & $<0$. & 0. & $<0.02$ & 0. & $<0$. & 0. & $<0.02$ & 0.54 \\
\hline $12 \mathrm{R}-2,25-28$ & 6 & 55.89 & & 0.3 & 0.9 & 0.17 & 1.0 & 0.34 & 0.2 & 0.3 & 0.05 & 0.32 & 0.06 & 0.18 & 0.03 & 0.19 & 0.03 & 1.9 \\
\hline $12 \mathrm{R}-4,53-5$ & 5 & 57.61 & & $<0.1$ & 0.1 & & 0.1 & & 0. & 0. & $<0.02$ & 0.05 & $<0.02$ & 0.02 & $<0.02$ & $<0.05$ & $<0.02$ & 0.18 \\
\hline $13 \mathrm{~W}-1,50-58$ & - & 15.00 & & $<0.1$ & 0.1 & 0.03 & 0.1 & 0.03 & 0.04 & 0.03 & $<0.02$ & 0.04 & $<0.02$ & $<0.02$ & $<0.02$ & $<0.05$ & $<0.02$ & 0.17 \\
\hline $13 \mathrm{~W}-1+2,0-150$ & - & 15.00 & & ND & ND & ND & ND & ND & ND & ND & ND & ND & ND & ND & ND & ND & ND & ND \\
\hline \multicolumn{19}{|l|}{ 158-95 } \\
\hline $1 \mathrm{R}-1,13-18$ & - & 0.13 & & ND & ND & ND & ND & ND & ND & ND & ND & ND & ND & ND & ND & ND & ND & ND \\
\hline $1 \mathrm{R}-3,70-100$ & - & $\begin{array}{l}3.75 \\
4.10\end{array}$ & & 0.3 & 0.8 & 0.11 & 0.6 & 0.10 & 0.23 & 0.08 & $<0.02$ & 0.06 & $<0.02$ & 0.03 & $<0.02$ & $<0.05$ & $<0.02$ & 0.31 \\
\hline $\begin{array}{l}1 \mathrm{R}-3,119-121 \\
2 \mathrm{R}-1,0-4\end{array}$ & $\overline{1}$ & $\begin{array}{l}4.10 \\
9.50\end{array}$ & & $\begin{array}{l}\mathrm{ND} \\
\mathrm{ND}\end{array}$ & $\begin{array}{l}\text { ND } \\
\text { ND }\end{array}$ & $\begin{array}{l}\mathrm{ND} \\
\mathrm{ND}\end{array}$ & $\begin{array}{l}\text { ND } \\
\text { ND }\end{array}$ & $\begin{array}{l}\mathrm{ND} \\
\mathrm{ND}\end{array}$ & $\begin{array}{l}\mathrm{ND} \\
\mathrm{ND}\end{array}$ & $\begin{array}{l}\mathrm{ND} \\
\mathrm{ND}\end{array}$ & $\begin{array}{l}\text { ND } \\
\text { ND }\end{array}$ & $\begin{array}{l}\text { ND } \\
\text { ND }\end{array}$ & $\begin{array}{l}\text { ND } \\
\text { ND }\end{array}$ & $\begin{array}{l}\text { ND } \\
\text { ND }\end{array}$ & $\begin{array}{l}\mathrm{ND} \\
\mathrm{ND}\end{array}$ & $\begin{array}{l}\mathrm{ND} \\
\mathrm{ND}\end{array}$ & $\begin{array}{l}\text { ND } \\
\text { ND }\end{array}$ & $\begin{array}{l}\text { ND } \\
\text { ND }\end{array}$ \\
\hline & & & & & & & & & & & & & & & & & & \\
\hline
\end{tabular}

Note: $\mathrm{ND}=$ element not determined; $<=$ values below reported detection. 
Table 6. Selected major and trace element compositions of pyrite and chalcopyrite in samples from Site 957.

\begin{tabular}{|c|c|c|c|c|c|c|c|c|c|c|c|}
\hline & & & & & & & $\begin{array}{l}\text { Element: } \\
\text { Method: }\end{array}$ & $\begin{array}{c}\mathrm{Fe} \\
\text { INAA }\end{array}$ & $\begin{array}{c}\mathrm{Zn} \\
\text { INAA }\end{array}$ & $\begin{array}{c}\mathrm{Au} \\
\text { INAA }\end{array}$ & $\begin{array}{c}\text { Ag } \\
\text { INAA }\end{array}$ \\
\hline $\begin{array}{l}\text { Core, section, } \\
\text { interval }(\mathrm{cm})\end{array}$ & Piece & $\begin{array}{l}\text { Depth } \\
\text { (mbsf) }\end{array}$ & Area & Lithology & $\begin{array}{c}\text { Grain } \\
\text { Size }\end{array}$ & Description & $\begin{array}{l}\text { Detection } \\
\text { limit: }\end{array}$ & $0.02 \%$ & $\begin{array}{c}50 \\
\mathrm{ppm}\end{array}$ & $\begin{array}{c}5 \\
\mathrm{ppb}\end{array}$ & $\begin{array}{c}5 \\
\mathrm{ppm}\end{array}$ \\
\hline Chalco & & & & & & & & & & & \\
\hline $\begin{array}{c}158-957 \mathrm{C}- \\
7 \mathrm{~N}-1,73-133 \\
7 \mathrm{~N}-2,0-75 \\
7 \mathrm{~N}-2,75-100 \\
11 \mathrm{~N}-1,30-97 \\
11 \mathrm{~N}-1,30-97 \\
11 \mathrm{~N}-2,0-30 \\
11 \mathrm{~N}-2,60-75 \\
11 \mathrm{~N}-3,75-134 \\
11 \mathrm{~N}-3,75-134 \\
12 \mathrm{~N}-2,0-67 \\
12 \mathrm{~N}-2,67-140 \\
12 \mathrm{~N}-3,0-100 \\
13 \mathrm{~N}-1,0-58 \\
13 \mathrm{~N}-2,0-62 \\
14 \mathrm{~N}-1,40-74 \\
14 \mathrm{~N}-2,0-63 \\
15 \mathrm{~N}-2,0-34 \\
15 \mathrm{~N}-2,34-150 \\
16 \mathrm{~N}-1,66-141\end{array}$ & $\begin{array}{c}7-8 \\
1 \mathrm{~A}-1 \mathrm{E} \\
1 \mathrm{~F}-1 \mathrm{~K} \\
3 \mathrm{~A}-3 \mathrm{H} \\
3 \mathrm{~A}-3 \mathrm{H} \\
1 \mathrm{~A}-1 \mathrm{D} \\
1 \mathrm{~J}-1 \mathrm{~K} \\
8-11 \\
8-11 \\
1-8 \\
9-17 \\
1-9 \\
1-10 \\
1-4 \\
6-10 \\
1-3 \\
1 \mathrm{~A}-1 \mathrm{G} \\
2-8 \\
9-17\end{array}$ & $\begin{array}{l}20.22 \\
21.00 \\
22.00 \\
31.10 \\
31.10 \\
32.20 \\
32.75 \\
34.50 \\
34.50 \\
36.00 \\
36.30 \\
36.75 \\
37.50 \\
38.75 \\
40.75 \\
41.20 \\
43.25 \\
44.00 \\
47.10\end{array}$ & $\begin{array}{l}\text { TAG-1 } \\
\text { TAG-1 } \\
\text { TAG-1 } \\
\text { TAG-1 } \\
\text { TAG-1 } \\
\text { TAG-1 } \\
\text { TAG-1 } \\
\text { TAG-1 } \\
\text { TAG-1 } \\
\text { TAG-1 } \\
\text { TAG-1 } \\
\text { TAG-1 } \\
\text { TAG-1 } \\
\text { TAG-1 } \\
\text { TAG-1 } \\
\text { TAG-1 } \\
\text { TAG-1 } \\
\text { TAG-1 } \\
\text { TAG-1 }\end{array}$ & $\begin{array}{l}\text { Pyrite-anhydrite breccia } \\
\text { Pyrite-anhydrite breccia } \\
\text { Pyrite-anhydrite breccia } \\
\text { Pyrite-silica breccia } \\
\text { Pyrite-silica breccia } \\
\text { Pyrite-silica-anhydrite breccia } \\
\text { Pyrite-silica-anhydrite breccia } \\
\text { Pyrite-silica breccia } \\
\text { Pyrite-silica breccia } \\
\text { Pyrite-silica-anhydrite breccia } \\
\text { Pyrite-silica-anhydrite breccia } \\
\text { Pyrite-silica-anhydrite breccia } \\
\text { Pyrite-silica breccia } \\
\text { Pyrite-silica-anhydrite breccia } \\
\text { Pyrite-silica breccia } \\
\text { Pyrite-silica breccia } \\
\text { Silicified wallrock breccia } \\
\text { Silicified wallrock breccia } \\
\text { Silicified wallrock breccia }\end{array}$ & $\begin{array}{l}\text { Fine } \\
\text { Fine } \\
\text { Fine } \\
\text { Fine } \\
\text { Fine } \\
\text { Fine } \\
\text { Fine } \\
\text { Fine } \\
\text { Fine } \\
\text { Fine } \\
\text { Fine } \\
\text { Fine } \\
\text { Fine } \\
\text { Fine } \\
\text { Fine } \\
\text { Fine } \\
\text { Fine } \\
\text { Fine } \\
\text { Fine }\end{array}$ & $\begin{array}{l}\text { Fragment adjacent to anhydrite vein } \\
\text { Intergrown with pyrite fragments } \\
\text { Adjacent to anhydrite vein } \\
\text { In massive anhydrite } \\
\text { Pyrite-chalcopyrite vein selvage } \\
\text { Replacing nodular pyrite } \\
\text { Vein-related, replacing pyrite } \\
\text { Chalcopyrite-pyrite vein-related } \\
\text { Massive chalcopyrite } \\
\text { In anhydrite vein } \\
\text { In massive anhydrite } \\
\text { In massive anhydrite } \\
\text { In anhydrite vein } \\
\text { Selvage of anhydrite vein } \\
\text { In anhydrite vein } \\
\text { In anhydrite vein } \\
\text { Pyrite-anhydrite vein in silica } \\
\text { Pyrite-anhydrite vein in silica } \\
\text { In anhydrite vein and selvage }\end{array}$ & & $\begin{array}{l}32.7 \\
37.1 \\
33.4 \\
28.0 \\
35.7 \\
37.3 \\
37.3 \\
42.0 \\
34.5 \\
33.7 \\
31.1 \\
35.1 \\
38.9 \\
34.4 \\
31.3 \\
33.0 \\
30.2 \\
29.3 \\
42.1\end{array}$ & $\begin{array}{r}540 \\
<50 \\
320 \\
85 \\
200 \\
240 \\
270 \\
1000 \\
<50 \\
<50 \\
210 \\
220 \\
440 \\
60 \\
<50 \\
85 \\
<50 \\
95 \\
<50\end{array}$ & $\begin{array}{r}69 \\
82 \\
55 \\
52 \\
77 \\
126 \\
109 \\
263 \\
64 \\
97 \\
66 \\
74 \\
41 \\
44 \\
55 \\
57 \\
<5 \\
42 \\
85\end{array}$ & $\begin{array}{l}<5 \\
<5 \\
<5 \\
<5 \\
<5 \\
<5 \\
<5 \\
<5 \\
<5 \\
<5 \\
<5 \\
<5 \\
<5 \\
<5 \\
<5 \\
<5 \\
<5 \\
<5 \\
<5\end{array}$ \\
\hline $\begin{array}{l}\text { 158-957E- } \\
\text { 1R-1, 0-20 } \\
\text { 3R-1,0-21 } \\
6 \mathrm{R}-1,0-40 \\
\text { 9R-1,0-27 }\end{array}$ & $\begin{array}{l}1-3 \\
1-4 \\
1-8 \\
1-5\end{array}$ & $\begin{array}{l}31.60 \\
41.75 \\
63.50 \\
78.00\end{array}$ & $\begin{array}{l}\text { TAG-1 } \\
\text { TAG-1 } \\
\text { TAG-1 } \\
\text { TAG-1 }\end{array}$ & $\begin{array}{l}\text { Pyrite-silica breccia } \\
\text { Pyrite-silica breccia } \\
\text { Pyrite-silica breccia } \\
\text { Silicified wallrock breccia }\end{array}$ & $\begin{array}{l}\text { Fine } \\
\text { Fine } \\
\text { Fine } \\
\text { Fine }\end{array}$ & $\begin{array}{l}\text { Selvage of anhydrite vein } \\
\text { Chalcopyrite-pyrite, vein-related } \\
\text { Massive pyrite-chalcopyrite } \\
\text { Massive pyrite-chalcopyrite }\end{array}$ & & $\begin{array}{l}41.0 \\
39.6 \\
49.9 \\
49.7\end{array}$ & $\begin{array}{l}<50 \\
130 \\
400 \\
310\end{array}$ & $\begin{array}{r}65 \\
138 \\
472 \\
238\end{array}$ & $\begin{array}{l}<5 \\
<5 \\
<5 \\
<5\end{array}$ \\
\hline $\begin{array}{l}158-957 \mathrm{~F}- \\
1 \mathrm{~N}-1,0-30 \\
1 \mathrm{~N}-1,31-74\end{array}$ & $\begin{array}{c}1-7 \\
8-10\end{array}$ & $\begin{array}{l}0.75 \\
1.35\end{array}$ & $\begin{array}{l}\text { TAG-1 } \\
\text { TAG-1 }\end{array}$ & $\begin{array}{l}\text { Massive pyrite breccia } \\
\text { Massive pyrite breccia }\end{array}$ & $\begin{array}{l}\text { Fine } \\
\text { Fine }\end{array}$ & $\begin{array}{l}\text { Massive chalcopyrite } \\
\text { Fragment in sandy pyrite }\end{array}$ & & $\begin{array}{l}32.2 \\
40.5\end{array}$ & $\begin{array}{r}1200 \\
170\end{array}$ & $\begin{array}{l}335 \\
299\end{array}$ & $\begin{array}{l}<5 \\
<5\end{array}$ \\
\hline $\begin{array}{l}\text { Pyrite: } \\
\text { 158-957B- } \\
\text { 1R-2, 0-10 }\end{array}$ & 1 & 1.03 & TAG-2 & Massive pyrite breccia & Medium & Massive, granular pyrite & & 4.56 & 150 & 71 & 5 \\
\hline $\begin{array}{l}158-957 \mathrm{C}- \\
4 \mathrm{~W}-1,3-8 \\
5 \mathrm{~N}-1,0-25 \\
7 \mathrm{~N}-1,0-69 \\
7 \mathrm{~N}-1,0-69 \\
7 \mathrm{~N}-1,73-133 \\
7 \mathrm{~N}-2,0-75 \\
7 \mathrm{~N}-2,75-100 \\
7 \mathrm{~N}-3,30-66 \\
8 \mathrm{~W}-1,0-5 \\
9 \mathrm{X}-1,4-10 \\
10 \mathrm{~N}-1,17-27 \\
11 \mathrm{~N}-1,0-30 \\
11 \mathrm{~N}-1,30-97 \\
11 \mathrm{~N}-2,0-30 \\
11 \mathrm{~N}-2,30-60 \\
11 \mathrm{~N}-2,60-75 \\
11 \mathrm{~N}-3,0-57 \\
12 \mathrm{~N}-1,0-68 \\
12 \mathrm{~N}-2,0-67 \\
12 \mathrm{~N}-2,67-140 \\
12 \mathrm{~N}-3,0-100 \\
13 \mathrm{~N}-1,0-58 \\
13 \mathrm{~N}-1,58-140 \\
13 \mathrm{~N}-2,0-62 \\
14 \mathrm{~N}-1,40-74 \\
14 \mathrm{~N}-2,0-63 \\
15 \mathrm{~N}-1,0-55 \\
15 \mathrm{~N}-1,55-120 \\
15 \mathrm{~N}-2,0-34 \\
15 \mathrm{~N}-2,34-150 \\
15 \mathrm{~N}-3,0-120 \\
15 \mathrm{~N}-3,120-150 \\
15 \mathrm{~N}-4,0-51 \\
16 \mathrm{~N}-1,4-59 \\
16 \mathrm{~N}-1,66-141 \\
16 \mathrm{~N}-2,0-88\end{array}$ & $\begin{array}{c}2 \\
1-4 \\
1-6 \mathrm{G} \\
1-6 \mathrm{G} \\
7-8 \\
1 \mathrm{~A}-1 \mathrm{E} \\
1 \mathrm{~F}-1 \mathrm{~K} \\
4 \mathrm{~A}-4 \mathrm{D} \\
1 \\
2 \\
4 \mathrm{~A}-4 \mathrm{~B} \\
1-3 \mathrm{~A} \\
3 \mathrm{~A}-3 \mathrm{H} \\
1 \mathrm{~A}-1 \mathrm{D} \\
1 \mathrm{E}-1 \mathrm{I} \\
1 \mathrm{~J}-1 \mathrm{~K} \\
1-5 \mathrm{C} \\
1-6 \\
1-8 \\
9-17 \\
1-9 \\
1-10 \\
11-22 \\
1-4 \\
6-10 \\
1-3 \\
1-7 \\
8-11 \\
1 \mathrm{~A}-1 \mathrm{G} \\
2-8 \\
1-9 \\
10-11 \\
1-4 \\
2-7 \\
9-17 \\
1-8\end{array}$ & $\begin{array}{l}10.52 \\
15.00 \\
19.55 \\
19.55 \\
20.22 \\
21.00 \\
22.00 \\
22.85 \\
19.50 \\
24.40 \\
28.86 \\
30.80 \\
31.10 \\
32.20 \\
32.50 \\
32.75 \\
33.75 \\
35.35 \\
36.00 \\
36.30 \\
36.75 \\
37.50 \\
38.00 \\
38.75 \\
40.75 \\
41.20 \\
42.50 \\
42.75 \\
43.25 \\
44.00 \\
44.75 \\
45.50 \\
45.90 \\
46.50 \\
47.10 \\
48.00\end{array}$ & $\begin{array}{l}\text { TAG-1 } \\
\text { TAG-1 } \\
\text { TAG-1 } \\
\text { TAG-1 } \\
\text { TAG-1 } \\
\text { TAG-1 } \\
\text { TAG-1 } \\
\text { TAG-1 } \\
\text { TAG-1 } \\
\text { TAG-1 } \\
\text { TAG-1 } \\
\text { TAG-1 } \\
\text { TAG-1 } \\
\text { TAG-1 } \\
\text { TAG-1 } \\
\text { TAG-1 } \\
\text { TAG-1 } \\
\text { TAG-1 } \\
\text { TAG-1 } \\
\text { TAG-1 } \\
\text { TAG-1 } \\
\text { TAG-1 } \\
\text { TAG-1 } \\
\text { TAG-1 } \\
\text { TAG-1 } \\
\text { TAG-1 } \\
\text { TAG-1 } \\
\text { TAG-1 } \\
\text { TAG-1 } \\
\text { TAG-1 } \\
\text { TAG-1 } \\
\text { TAG-1 } \\
\text { TAG-1 } \\
\text { TAG-1 } \\
\text { TAG-1 } \\
\text { TAG-1 }\end{array}$ & $\begin{array}{l}\text { Porous nodular pyrite breccia } \\
\text { Pyrite-anhydrite breccia } \\
\text { Pyrite-anhydrite breccia } \\
\text { Pyrite-anhydrite breccia } \\
\text { Pyrite-anhydrite breccia } \\
\text { Pyrite-anhydrite breccia } \\
\text { Pyrite-anhydrite breccia } \\
\text { Pyrite-anhydrite breccia } \\
\text { Pyrite-anhydrite breccia } \\
\text { Pyrite-anhydrite breccia } \\
\text { Pyrite-anhydrite breccia } \\
\text { Pyrite-silica-anhydrite breccia } \\
\text { Pyrite-silica breccia } \\
\text { Pyrite-silica-anhydrite breccia } \\
\text { Pyrite-silica-anhydrite breccia } \\
\text { Pyrite-silica-anhydrite breccia } \\
\text { Pyrite-silica-anhydrite breccia } \\
\text { Pyrite-silica-anhydrite breccia } \\
\text { Pyrite-silica-anhydrite breccia } \\
\text { Pyrite-silica breccia } \\
\text { Pyrite-silica-anhydrite breccia } \\
\text { Pyrite-silica breccia } \\
\text { Pyrite-silica-anhydrite breccia } \\
\text { Pyrite-silica-anhydrite breccia } \\
\text { Pyrite-silica breccia } \\
\text { Pyrite-silica breccia } \\
\text { Pyrite-silica breccia } \\
\text { Silicified wallrock breccia } \\
\text { Silicified wallrock breccia } \\
\text { Silicified wallrock breccia } \\
\text { Silicified wallrock breccia } \\
\text { Silicified wallrock breccia } \\
\text { Silicified wallrock breccia } \\
\text { Silicified wallrock breccia } \\
\text { Silicified wallrock breccia } \\
\text { Silicified wallrock breccia }\end{array}$ & $\begin{array}{l}\text { Medium } \\
\text { Medium } \\
\text { Medium } \\
\text { Medium } \\
\text { Medium } \\
\text { Coarse } \\
\text { Medium } \\
\text { Coarse } \\
\text { Medium } \\
\text { Medium } \\
\text { Coarse } \\
\text { Medium } \\
\text { Medium } \\
\text { Coarse } \\
\text { Medium } \\
\text { Medium } \\
\text { Medium } \\
\text { Medium } \\
\text { Medium } \\
\text { Medium } \\
\text { Medium } \\
\text { Fine } \\
\text { Medium } \\
\text { Medium } \\
\text { Medium } \\
\text { Medium } \\
\text { Medium } \\
\text { Medium } \\
\text { Medium } \\
\text { Medium } \\
\text { Medium } \\
\text { Medium } \\
\text { Coarse } \\
\text { Coarse } \\
\text { Medium } \\
\text { Medium }\end{array}$ & $\begin{array}{l}\text { Nodular pyrite } \\
\text { Nodular pyrite } \\
\text { Halo of anhydrite vein } \\
\text { Nodular pyrite } \\
\text { Nodular pyrite } \\
\text { clast or fragment } \\
\text { Nodular pyrite } \\
\text { Nodular pyrite } \\
\text { Nodular pyrite } \\
\text { Nodular pyrite } \\
\text { Granular, euhedral aggregates } \\
\text { Nodular pyrite } \\
\text { Nodular pyrite } \\
\text { Nodular pyrite } \\
\text { Granular, euhedral aggregates } \\
\text { Granular, euhedral, vein-related } \\
\text { Nodular pyrite, vein-related } \\
\text { Granular, euhedral aggregates } \\
\text { Nodular pyrite } \\
\text { Granular, euhedral aggregates } \\
\text { Granular, euhedral aggregates } \\
\text { Halo of anhydrite vein } \\
\text { Granular, euhedral aggregates } \\
\text { Granular, euhedral aggregates } \\
\text { Brecciated, pyrite, silica, anhydrite } \\
\text { Brecciated, pyrite, silica, anhydrite } \\
\text { Brecciated, massive pyrite } \\
\text { Adjacent to thin anhydrite vein } \\
\text { Nodular pyrite, silica, anhydrite } \\
\text { Nodular pyrite, silica, anhydrite } \\
\text { Halo of anhydrite vein } \\
\text { Brecciated, pyrite-anhydrite } \\
\text { Nodular pyrite } \\
\text { Nodular pyrite } \\
\text { Brecciated halo of anhydrite vein } \\
\text { Brecciated halo of anhydrite vein }\end{array}$ & & $\begin{array}{l}43.4 \\
45.2 \\
33.8 \\
43.7 \\
42.1 \\
44.1 \\
43.5 \\
41.3 \\
41.2 \\
41.1 \\
40.2 \\
43.2 \\
39.3 \\
39.1 \\
40.2 \\
43.3 \\
45.2 \\
44.2 \\
44.1 \\
43.9 \\
39.3 \\
43.7 \\
40.4 \\
39.0 \\
39.3 \\
42.3 \\
39.1 \\
42.7 \\
37.7 \\
48.1 \\
45.3 \\
49.4 \\
49.2 \\
53.6 \\
51.2 \\
51.5\end{array}$ & $\begin{array}{r}750 \\
310 \\
660 \\
300 \\
1000 \\
240 \\
880 \\
290 \\
370 \\
630 \\
290 \\
390 \\
490 \\
480 \\
330 \\
700 \\
940 \\
100 \\
510 \\
190 \\
360 \\
640 \\
560 \\
190 \\
670 \\
1100 \\
1100 \\
830 \\
180 \\
620 \\
400 \\
630 \\
130 \\
<50 \\
790 \\
150\end{array}$ & $\begin{array}{r}300 \\
342 \\
80 \\
153 \\
177 \\
168 \\
229 \\
187 \\
152 \\
125 \\
138 \\
84 \\
97 \\
139 \\
183 \\
164 \\
168 \\
131 \\
225 \\
117 \\
143 \\
160 \\
171 \\
150 \\
144 \\
151 \\
176 \\
350 \\
48 \\
149 \\
92 \\
146 \\
23 \\
58 \\
231 \\
110\end{array}$ & $\begin{array}{l}5 \\
<5 \\
<5 \\
<5 \\
<5 \\
<5 \\
<5 \\
<5 \\
<5 \\
<5 \\
<5 \\
<5 \\
<5 \\
<5 \\
<5 \\
<5 \\
<5 \\
<5 \\
<5 \\
<5 \\
<5 \\
<5 \\
<5 \\
<5 \\
<5 \\
<5 \\
<5 \\
<5 \\
<5 \\
<5 \\
<5 \\
<5 \\
<5 \\
<5 \\
<5 \\
<5\end{array}$ \\
\hline $\begin{array}{l}1 \mathrm{R}-1,0-20 \\
2 \mathrm{R}-1,0-42 \\
4 \mathrm{R}-1,0-23 \\
5 \mathrm{R}-1,0-44 \\
5 \mathrm{R}-1,0-44 \\
7 \mathrm{R}-1,0-33 \\
10 \mathrm{R}-1,0-16 \\
11 \mathrm{R}-1,0-54 \\
12 \mathrm{R}-1,0-46 \\
14 \mathrm{R}-1,0-54 \\
15 \mathrm{R}-1,0-50\end{array}$ & $\begin{array}{c}1-3 \\
1-7 \\
1-4 \\
1-8 \\
1-8 \\
1-6 \\
1-2 \\
1-10 \\
1-7 \\
1-11 \\
1-9\end{array}$ & $\begin{array}{r}31.60 \\
37.25 \\
49.10 \\
58.75 \\
58.75 \\
68.45 \\
82.15 \\
87.30 \\
92.00 \\
101.75 \\
106.75\end{array}$ & $\begin{array}{l}\text { TAG-1 } \\
\text { TAG-1 } \\
\text { TAG-1 } \\
\text { TAG-1 } \\
\text { TAG-1 } \\
\text { TAG-1 } \\
\text { TAG-1 } \\
\text { TAG-1 } \\
\text { TAG-1 } \\
\text { TAG-1 } \\
\text { TAG-1 }\end{array}$ & $\begin{array}{l}\text { Pyrite-silica breccia } \\
\text { Pyrite-silica breccia } \\
\text { Pyrite-silica breccia } \\
\text { Pyrite-silica breccia } \\
\text { Pyrite-silica breccia } \\
\text { Pyrite-silica breccia } \\
\text { Silicified wallrock breccia } \\
\text { Pyrite-silica breccia } \\
\text { Silicified wallrock breccia } \\
\text { Pyrite-silica breccia } \\
\text { Chloritized basalt breccia }\end{array}$ & $\begin{array}{l}\text { Coarse } \\
\text { Coarse } \\
\text { Coarse } \\
\text { Medium } \\
\text { Fine } \\
\text { Coarse } \\
\text { Medium } \\
\text { Medium } \\
\text { Medium } \\
\text { Fine } \\
\text { Medium }\end{array}$ & $\begin{array}{l}\text { Granular, euhedral aggregates } \\
\text { Granular, euhedral aggregates } \\
\text { Granular, euhedral aggregates } \\
\text { Granular, euhedral aggregates } \\
\text { Clasts or fragments } \\
\text { Nodular pyrite } \\
\text { Massive pyrite+chalcopyrite } \\
\text { Halo of anhydrite vein } \\
\text { Halo of anhydrite vein } \\
\text { Disseminated matrix pyrite } \\
\text { Clasts or fragments }\end{array}$ & & $\begin{array}{l}47.2 \\
52.6 \\
49.2 \\
53.1 \\
46.6 \\
50.4 \\
51.5 \\
46.2 \\
49.9 \\
47.1 \\
50.2\end{array}$ & $\begin{array}{r}280 \\
220 \\
300 \\
210 \\
120 \\
25 \\
110 \\
410 \\
470 \\
160 \\
210\end{array}$ & $\begin{array}{r}234 \\
113 \\
340 \\
139 \\
387 \\
51 \\
203 \\
332 \\
258 \\
149 \\
238\end{array}$ & $\begin{array}{l}<5 \\
<5 \\
<5 \\
<5 \\
<5 \\
<5 \\
<5 \\
<5 \\
<5 \\
<5 \\
<5 \\
<5\end{array}$ \\
\hline
\end{tabular}


Table 6 (continued).

\begin{tabular}{|c|c|c|c|c|c|c|c|c|c|c|c|c|c|c|}
\hline & $\begin{array}{l}\text { Element: } \\
\text { Method: }\end{array}$ & $\begin{array}{c}\text { As } \\
\text { INAA }\end{array}$ & $\begin{array}{c}\mathrm{Sb} \\
\text { INAA }\end{array}$ & $\begin{array}{c}\text { Mo } \\
\text { INAA }\end{array}$ & $\begin{array}{c}\text { Co } \\
\text { INAA }\end{array}$ & $\begin{array}{c}\mathrm{Se} \\
\text { INAA }\end{array}$ & $\begin{array}{c}\mathrm{Ni} \\
\text { INAA }\end{array}$ & $\begin{array}{c}\mathrm{Ca} \\
\text { INAA }\end{array}$ & $\begin{array}{c}\mathrm{Sr} \\
\text { INAA }\end{array}$ & $\begin{array}{c}\mathrm{Rb} \\
\text { INAA }\end{array}$ & $\begin{array}{c}\mathrm{Na} \\
\text { INAA }\end{array}$ & $\begin{array}{c}\mathrm{Br} \\
\text { INAA }\end{array}$ & $\begin{array}{c}\text { U } \\
\text { INAA }\end{array}$ & $\begin{array}{c}\mathrm{Zn} \\
\text { INAA }\end{array}$ \\
\hline $\begin{array}{l}\text { Core, section, } \\
\text { interval }(\mathrm{cm})\end{array}$ & $\begin{array}{l}\text { Detection } \\
\text { limit: }\end{array}$ & $\begin{array}{c}2 \\
\mathrm{ppm}\end{array}$ & $\begin{array}{c}0.2 \\
\mathrm{ppm}\end{array}$ & $\begin{array}{c}5 \\
\mathrm{ppm}\end{array}$ & $\begin{array}{c}5 \\
\mathrm{ppm}\end{array}$ & $\begin{array}{c}5 \\
\mathrm{ppm}\end{array}$ & $\begin{array}{c}5 \\
\mathrm{ppm}\end{array}$ & $\begin{array}{c}50 \\
\mathrm{ppm}\end{array}$ & $1 \%$ & $\begin{array}{l}500 \\
\mathrm{ppm}\end{array}$ & $\begin{array}{c}30 \\
\mathrm{ppm}\end{array}$ & $\begin{array}{l}500 \\
\text { ppm }\end{array}$ & $\begin{array}{c}1 \\
\mathrm{ppm}\end{array}$ & $\begin{array}{c}0.5 \\
\mathrm{ppm}\end{array}$ \\
\hline Chalcopyrite: & & & & & & & & & & & & & & \\
\hline $\begin{array}{c}158-957 \mathrm{C}- \\
7 \mathrm{~N}-1,73-133 \\
7 \mathrm{~N}-2,0-75 \\
7 \mathrm{~N}-2,75-100 \\
11 \mathrm{~N}-1,30-97 \\
11 \mathrm{~N}-1,30-97 \\
11 \mathrm{~N}-2,0-30 \\
11 \mathrm{~N}-2,60-75 \\
11 \mathrm{~N}-3,75-134 \\
11 \mathrm{~N}-3,75-134 \\
12 \mathrm{~N}-2,0-67 \\
12 \mathrm{~N}-2,67-140 \\
12 \mathrm{~N}-3,0-100 \\
13 \mathrm{~N}-1,0-58 \\
13 \mathrm{~N}-2,0-62 \\
14 \mathrm{~N}-1,40-74 \\
14 \mathrm{~N}-2,0-63 \\
15 \mathrm{~N}-2,0-34 \\
15 \mathrm{~N}-2,34-150 \\
16 \mathrm{~N}-1,66-141\end{array}$ & & $\begin{array}{r}10 \\
25 \\
12 \\
<2 \\
21 \\
21 \\
20 \\
44 \\
10 \\
8 \\
8 \\
16 \\
18 \\
8 \\
4 \\
5 \\
<2 \\
3 \\
14\end{array}$ & $\begin{array}{r}<0.2 \\
<0.2 \\
<0.2 \\
<0.2 \\
0.4 \\
0.5 \\
<0.2 \\
1.3 \\
0.5 \\
<0.2 \\
<0.2 \\
0.4 \\
0.9 \\
0.3 \\
<0.2 \\
<0.2 \\
<0.2 \\
<0.2 \\
<0.2\end{array}$ & $\begin{array}{r}16 \\
56 \\
26 \\
38 \\
120 \\
95 \\
110 \\
180 \\
79 \\
140 \\
60 \\
71 \\
150 \\
110 \\
67 \\
46 \\
<5 \\
31 \\
49\end{array}$ & $\begin{array}{r}67 \\
190 \\
150 \\
120 \\
140 \\
170 \\
190 \\
190 \\
150 \\
210 \\
200 \\
170 \\
220 \\
260 \\
240 \\
160 \\
160 \\
140 \\
250\end{array}$ & $\begin{array}{c}24 \\
<5 \\
37 \\
140 \\
16 \\
17 \\
24 \\
13 \\
2.5 \\
31 \\
68 \\
25 \\
30 \\
38 \\
48 \\
45 \\
29 \\
30 \\
35\end{array}$ & $\begin{array}{r}80 \\
120 \\
70 \\
50 \\
<50 \\
<50 \\
<50 \\
<50 \\
70 \\
70 \\
<50 \\
<50 \\
70 \\
<50 \\
<50 \\
<50 \\
90 \\
60 \\
70\end{array}$ & $\begin{array}{l}2 \\
3 \\
1.5 \\
1 \\
1 \\
1 \\
2 \\
1 \\
1.5 \\
1.5 \\
3 \\
1 \\
1.5 \\
1 \\
1 \\
1 \\
1 \\
1 \\
3\end{array}$ & $\begin{array}{l}<500 \\
<500 \\
<500 \\
<500 \\
<500 \\
<500 \\
<500 \\
<500 \\
<500 \\
<500 \\
<500 \\
<500 \\
<500 \\
<500 \\
<500 \\
<500 \\
<500 \\
450 \\
650\end{array}$ & $\begin{array}{l}<30 \\
<30 \\
<30 \\
<30 \\
<30 \\
<30 \\
<30 \\
<30 \\
<30 \\
<30 \\
<30 \\
<30 \\
90 \\
<30 \\
<30 \\
<30 \\
<30 \\
<30 \\
<30\end{array}$ & $\begin{array}{l}230 \\
660 \\
410 \\
380 \\
210 \\
290 \\
200 \\
180 \\
350 \\
690 \\
320 \\
410 \\
370 \\
230 \\
660 \\
180 \\
180 \\
570 \\
440\end{array}$ & $\begin{array}{r}6 \\
12 \\
5 \\
5 \\
<1 \\
3 \\
2 \\
<1 \\
5 \\
7 \\
4 \\
<1 \\
7 \\
<1 \\
<1 \\
5 \\
<1 \\
<1 \\
5 \\
7\end{array}$ & $\begin{array}{r}5.0 \\
0.7 \\
6.4 \\
<0.5 \\
<0.5 \\
3.7 \\
<0.5 \\
4.0 \\
<0.5 \\
0.4 \\
<0.5 \\
2.5 \\
<0.5 \\
<0.5 \\
<0.5 \\
<0.5 \\
2.7 \\
<0.5 \\
<0.5\end{array}$ & $\begin{array}{l}<0.5 \\
<0.5 \\
<0.5 \\
<0.5 \\
<0.5 \\
<0.5 \\
<0.5 \\
<0.5 \\
<0.5 \\
<0.5 \\
<0.5 \\
<0.5 \\
<0.5 \\
<0.5 \\
<0.5 \\
<0.5 \\
<0.5 \\
<0.5 \\
<0.5\end{array}$ \\
\hline $\begin{array}{l}\text { 158-957E- } \\
\text { 1R-1, 0-20 } \\
\text { 3R-1, 0-21 } \\
\text { 6R-1, 0-40 } \\
\text { 9R-1, 0-27 }\end{array}$ & & $\begin{array}{l}10 \\
18 \\
64 \\
46\end{array}$ & $\begin{array}{r}<0.2 \\
0.9 \\
0.4 \\
1.1\end{array}$ & $\begin{array}{r}200 \\
90 \\
96 \\
120\end{array}$ & $\begin{array}{l}230 \\
150 \\
160 \\
190\end{array}$ & $\begin{array}{l}<5 \\
28 \\
<5 \\
<5\end{array}$ & $\begin{array}{l}80 \\
80 \\
80 \\
60\end{array}$ & $\begin{array}{l}1.5 \\
1.5 \\
1.5 \\
1\end{array}$ & $\begin{array}{r}700 \\
700 \\
<500 \\
<500\end{array}$ & $\begin{array}{l}<30 \\
<30 \\
<30 \\
<30\end{array}$ & $\begin{array}{r}1580 \\
680 \\
1150 \\
780\end{array}$ & $\begin{array}{r}10 \\
11 \\
9 \\
8\end{array}$ & $\begin{array}{l}<0.5 \\
9.3 \\
12 \\
<0.5\end{array}$ & $\begin{array}{l}<0.5 \\
<0.5 \\
<0.5 \\
<0.5\end{array}$ \\
\hline $\begin{array}{l}158-957 \mathrm{~F}- \\
1 \mathrm{~N}-1,0-30 \\
1 \mathrm{~N}-1,31-74\end{array}$ & & $\begin{array}{r}8 \\
19\end{array}$ & $\begin{array}{l}1.1 \\
0.5\end{array}$ & $\begin{array}{l}210 \\
120\end{array}$ & $\begin{array}{l}180 \\
320\end{array}$ & $\begin{array}{r}1000 \\
900\end{array}$ & $\begin{array}{r}40 \\
110\end{array}$ & $\begin{array}{l}<1 \\
1.5\end{array}$ & $\begin{array}{r}500 \\
1200\end{array}$ & $\begin{array}{l}<30 \\
<30\end{array}$ & $\begin{array}{r}1110 \\
670\end{array}$ & $\begin{array}{r}7 \\
17\end{array}$ & $\begin{array}{r}<0.5 \\
0.6\end{array}$ & $\begin{array}{r}1.6 \\
<0.5\end{array}$ \\
\hline Pyrite: & & & & & & & & & & & & & & \\
\hline $\begin{array}{l}\text { 158-957B- } \\
1 \mathrm{R}-2,0-10\end{array}$ & & 17 & $<0.2$ & 92 & 240 & $<5$ & 80 & 2 & $<500$ & $<30$ & 260 & $<1$ & $<0.5$ & $<0.5$ \\
\hline $\begin{array}{l}158-957 \mathrm{C}- \\
4 \mathrm{~W}-1,3-8 \\
5 \mathrm{~N}-1,0-25 \\
7 \mathrm{~N}-1,0-69 \\
7 \mathrm{~N}-1,0-69 \\
7 \mathrm{~N}-1,73-133 \\
7 \mathrm{~N}-2,0-75 \\
7 \mathrm{~N}-2,75-100 \\
7 \mathrm{~N}-3,30-66 \\
8 \mathrm{~W}-1,0-5 \\
9 \mathrm{X}-1,4-10 \\
10 \mathrm{~N}-1,17-27 \\
11 \mathrm{~N}-1,0-30 \\
11 \mathrm{~N}-1,30-97 \\
11 \mathrm{~N}-2,0-30 \\
11 \mathrm{~N}-2,30-60 \\
11 \mathrm{~N}-2,60-75 \\
11 \mathrm{~N}-3,0-57 \\
12 \mathrm{~N}-1,0-68 \\
12 \mathrm{~N}-2,0-67 \\
12 \mathrm{~N}-2,67-140 \\
12 \mathrm{~N}-3,0-100 \\
13 \mathrm{~N}-1,0-58 \\
13 \mathrm{~N}-1,58-140 \\
13 \mathrm{~N}-2,0-62 \\
14 \mathrm{~N}-1,40-74 \\
14 \mathrm{~N}-2,0-63 \\
15 \mathrm{~N}-1,0-55 \\
15 \mathrm{~N}-1,55-120 \\
15 \mathrm{~N}-2,0-34 \\
15 \mathrm{~N}-2,34-150 \\
15 \mathrm{~N}-3,0-120 \\
15 \mathrm{~N}-3,120-150 \\
15 \mathrm{~N}-4,0-51 \\
16 \mathrm{~N}-1,4-59 \\
16 \mathrm{~N}-1,66-141 \\
16 \mathrm{~N}-2,0-88\end{array}$ & & $\begin{array}{l}51 \\
57 \\
13 \\
40 \\
54 \\
44 \\
38 \\
42 \\
36 \\
36 \\
36 \\
29 \\
29 \\
34 \\
42 \\
30 \\
44 \\
24 \\
33 \\
43 \\
40 \\
34 \\
52 \\
41 \\
34 \\
47 \\
59 \\
52 \\
16 \\
53 \\
35 \\
38 \\
19 \\
36 \\
58 \\
43\end{array}$ & $\begin{array}{r}1.8 \\
0.4 \\
<0.2 \\
0.8 \\
1.0 \\
0.8 \\
0.7 \\
0.9 \\
<0.2 \\
<0.2 \\
0.4 \\
<0.2 \\
0.5 \\
0.8 \\
0.7 \\
0.7 \\
<0.2 \\
0.4 \\
0.6 \\
0.6 \\
0.6 \\
1.1 \\
1.0 \\
0.7 \\
0.6 \\
1.0 \\
0.9 \\
0.9 \\
0.7 \\
1.0 \\
0.6 \\
0.6 \\
<0.2 \\
<0.2 \\
1.7 \\
<0.2\end{array}$ & $\begin{array}{r}120 \\
67 \\
70 \\
93 \\
190 \\
82 \\
140 \\
85 \\
110 \\
110 \\
71 \\
68 \\
150 \\
110 \\
98 \\
150 \\
150 \\
140 \\
120 \\
95 \\
76 \\
200 \\
120 \\
84 \\
83 \\
80 \\
130 \\
120 \\
56 \\
97 \\
80 \\
93 \\
58 \\
35 \\
100 \\
41\end{array}$ & $\begin{array}{r}400 \\
280 \\
94 \\
420 \\
170 \\
300 \\
210 \\
400 \\
310 \\
240 \\
520 \\
450 \\
210 \\
150 \\
310 \\
300 \\
330 \\
410 \\
450 \\
260 \\
250 \\
160 \\
210 \\
210 \\
160 \\
180 \\
230 \\
200 \\
770 \\
270 \\
310 \\
240 \\
800 \\
830 \\
190 \\
1200\end{array}$ & $\begin{array}{l}28 \\
22 \\
53 \\
21 \\
<5 \\
17 \\
17 \\
20 \\
25 \\
20 \\
20 \\
31 \\
36 \\
18 \\
185 \\
25 \\
22 \\
23 \\
23 \\
36 \\
37 \\
17 \\
26 \\
10 \\
11 \\
11 \\
<5 \\
13 \\
<5 \\
16 \\
<5\end{array}$ & $\begin{array}{r}70 \\
50 \\
80 \\
60 \\
50 \\
50 \\
60 \\
<50 \\
<50 \\
60 \\
<50 \\
90 \\
<50 \\
50 \\
50 \\
<50 \\
50 \\
50 \\
50 \\
330 \\
<50 \\
<50 \\
<50 \\
<50 \\
<50 \\
440 \\
400 \\
510 \\
80 \\
<50 \\
50 \\
50 \\
60 \\
90 \\
60 \\
70\end{array}$ & $\begin{array}{l}2 \\
1 \\
2 \\
1.5 \\
1 \\
1 \\
1.5 \\
1 \\
1 \\
2 \\
1 \\
2 \\
1 \\
1.5 \\
1 \\
1 \\
2 \\
1 \\
1 \\
2 \\
2 \\
1 \\
<1 \\
1 \\
1 \\
1.5 \\
<1 \\
1 \\
1 \\
<1 \\
1 \\
1 \\
1 \\
1.5 \\
1 \\
1\end{array}$ & $\begin{array}{l}<500 \\
<500 \\
<500 \\
<500 \\
<500 \\
<500 \\
<500 \\
<500 \\
<500 \\
<500 \\
<500 \\
<500 \\
<500 \\
<500 \\
<500 \\
<500 \\
<500 \\
<500 \\
<500 \\
<500 \\
<500 \\
<500 \\
<500 \\
<500 \\
<500 \\
<500 \\
<500 \\
<500 \\
<500 \\
<500 \\
<500 \\
<500 \\
<500 \\
<500 \\
<500 \\
<500\end{array}$ & $\begin{array}{r}<30 \\
<30 \\
<30 \\
<30 \\
<30 \\
<30 \\
<30 \\
<30 \\
50 \\
<30 \\
<30 \\
<30 \\
<30 \\
<30 \\
<30 \\
<30 \\
<30 \\
<30 \\
<30 \\
<30 \\
<30 \\
<30 \\
<30 \\
<30 \\
<30 \\
<30 \\
50 \\
<30 \\
<30 \\
<30 \\
50 \\
<30 \\
<30 \\
<30 \\
<30 \\
<30\end{array}$ & $\begin{array}{l}570 \\
460 \\
790 \\
490 \\
650 \\
540 \\
330 \\
290 \\
430 \\
470 \\
280 \\
470 \\
420 \\
350 \\
300 \\
260 \\
400 \\
460 \\
760 \\
180 \\
470 \\
220 \\
230 \\
150 \\
390 \\
290 \\
290 \\
280 \\
280 \\
600 \\
880 \\
510 \\
490 \\
290 \\
200 \\
350\end{array}$ & $\begin{array}{r}4 \\
<1 \\
11 \\
4 \\
5 \\
4 \\
<1 \\
4 \\
<1 \\
<1 \\
<1 \\
<1 \\
3 \\
4 \\
3 \\
3 \\
4 \\
5 \\
4 \\
7 \\
<1 \\
5 \\
<1 \\
2 \\
<1 \\
3 \\
<1 \\
<1 \\
<1 \\
<1 \\
4 \\
5 \\
5 \\
5 \\
4 \\
7 \\
4 \\
5\end{array}$ & $\begin{array}{c}10 \\
11 \\
0.5 \\
5.7 \\
11 \\
13 \\
9.2 \\
7.9 \\
5.7 \\
0.5 \\
5.6 \\
0.5 \\
15 \\
2.7 \\
4.5 \\
1.9 \\
4.5 \\
4.0 \\
4.3 \\
<0.5 \\
3.2 \\
2.9 \\
2.8 \\
3.5 \\
3.9 \\
3.6 \\
4.6 \\
6.2 \\
<0.5 \\
4.9 \\
3.2 \\
3.1 \\
11 \\
<0.5 \\
7.6 \\
<0.5\end{array}$ & $\begin{array}{l}<0.5 \\
<0.5 \\
<0.5 \\
<0.5 \\
<0.5 \\
<0.5 \\
<0.5 \\
<0.5 \\
<0.5 \\
<0.5 \\
<0.5 \\
<0.5 \\
<0.5 \\
<0.5 \\
<0.5 \\
<0.5 \\
<0.5 \\
<0.5 \\
<0.5 \\
<0.5 \\
<0.5 \\
<0.5 \\
<0.5 \\
<0.5 \\
<0.5 \\
<0.5 \\
<0.5 \\
<0.5 \\
<0.5 \\
<0.5 \\
<0.5 \\
<0.5 \\
<0.5 \\
<0.5 \\
<0.5 \\
<0.5\end{array}$ \\
\hline $\begin{array}{c}\text { 158-957E- } \\
1 \mathrm{R}-1,0-20 \\
2 \mathrm{R}-1,0-42 \\
4 \mathrm{R}-1,0-23 \\
5 \mathrm{R}-1,0-44 \\
5 \mathrm{R}-1,0-44 \\
7 \mathrm{R}-1,0-33 \\
10 \mathrm{R}-1,0-16 \\
11 \mathrm{R}-1,0-54 \\
12 \mathrm{R}-1,0-46 \\
14 \mathrm{R}-1,0-54 \\
15 \mathrm{R}-1,0-50\end{array}$ & & $\begin{array}{l}53 \\
38 \\
63 \\
37 \\
46 \\
26 \\
21 \\
41 \\
45 \\
66 \\
29\end{array}$ & $\begin{array}{c}1.0 \\
0.7 \\
1.2 \\
0.8 \\
0.8 \\
0.7 \\
<0.2 \\
0.7 \\
0.7 \\
0.8 \\
0.7\end{array}$ & $\begin{array}{r}75 \\
120 \\
110 \\
61 \\
95 \\
42 \\
61 \\
120 \\
130 \\
130 \\
120\end{array}$ & $\begin{array}{r}260 \\
660 \\
470 \\
770 \\
450 \\
900 \\
67 \\
410 \\
300 \\
540 \\
710\end{array}$ & $\begin{array}{r}23 \\
77 \\
26 \\
46 \\
26 \\
65 \\
<5 \\
28 \\
25 \\
53 \\
130\end{array}$ & $\begin{array}{r}50 \\
80 \\
70 \\
100 \\
70 \\
80 \\
80 \\
60 \\
60 \\
60 \\
60\end{array}$ & $\begin{array}{l}3 \\
1 \\
1 \\
1.5 \\
1 \\
1 \\
1.5 \\
1 \\
2 \\
1 \\
1\end{array}$ & $\begin{array}{l}<500 \\
<500 \\
<500 \\
<500 \\
<500 \\
1200 \\
<500 \\
<500 \\
<500 \\
<500 \\
<500\end{array}$ & $\begin{array}{l}<30 \\
<30 \\
<30 \\
<30 \\
<30 \\
<30 \\
<30 \\
<30 \\
<30 \\
<30 \\
<30\end{array}$ & $\begin{array}{l}410 \\
780 \\
310 \\
420 \\
450 \\
810 \\
470 \\
210 \\
680 \\
550 \\
490\end{array}$ & $\begin{array}{r}5 \\
8 \\
6 \\
<1 \\
7 \\
7 \\
7 \\
3 \\
3 \\
< \\
5 \\
5\end{array}$ & $\begin{array}{c}7.3 \\
<0.5 \\
13 \\
0.25 \\
8.8 \\
<0.5 \\
<0.5 \\
<0.5 \\
5.9 \\
<0.5 \\
<0.5\end{array}$ & $\begin{array}{r}<0.5 \\
<0.5 \\
0.9 \\
<0.5 \\
<0.5 \\
<0.5 \\
<0.5 \\
<0.5 \\
<0.5 \\
<0.5 \\
<0.5\end{array}$ \\
\hline
\end{tabular}


Table 6 (continued).

\begin{tabular}{|c|c|c|c|c|c|c|c|c|c|c|c|}
\hline & & & & & & & $\begin{array}{l}\text { Element: } \\
\text { Method: }\end{array}$ & $\begin{array}{c}\mathrm{Fe} \\
\text { INAA }\end{array}$ & $\begin{array}{c}\mathrm{Zn} \\
\text { INAA }\end{array}$ & $\begin{array}{c}\mathrm{Au} \\
\text { INAA }\end{array}$ & $\begin{array}{c}\mathrm{Ag} \\
\text { INAA }\end{array}$ \\
\hline $\begin{array}{l}\text { Core, section, } \\
\text { interval }(\mathrm{cm})\end{array}$ & Piece & $\begin{array}{l}\text { Depth } \\
\text { (mbsf) }\end{array}$ & Area & Lithology & $\begin{array}{l}\text { Grain } \\
\text { Size }\end{array}$ & Description & $\begin{array}{l}\text { Detection } \\
\text { limit: }\end{array}$ & $0.02 \%$ & $\begin{array}{l}50 \\
\mathrm{ppm}\end{array}$ & $\begin{array}{c}5 \\
\mathrm{ppb}\end{array}$ & $\begin{array}{c}5 \\
\mathrm{ppm}\end{array}$ \\
\hline $15 \mathrm{R}-1,0-50$ & $1-9$ & 106.75 & TAG-1 & Chloritized basalt breccia & Medium & Clasts or fragments & & 50.2 & 210 & 238 & $<5$ \\
\hline $16 \mathrm{R}-1,0-20$ & $1-3$ & 111.15 & TAG-1 & Silicified wallrock breccia & Medium & Quartz-pyrite veins & & 41.3 & 460 & 82 & $<5$ \\
\hline $17 \mathrm{R}-1,0-47$ & $1-7$ & 116.20 & TAG-1 & Silicified wallrock breccia & Medium & Quartz-pyrite veins & & 45.7 & 250 & 95 & $<5$ \\
\hline $18 \mathrm{R}-1,0-20$ & $1-3$ & 120.80 & TAG-1 & Chloritized basalt breccia & Medium & Quartz-pyrite veins & & 48.8 & 600 & 202 & $<5$ \\
\hline \multicolumn{12}{|l|}{$158-957 \mathrm{~F}-$} \\
\hline $1 \mathrm{~N}-1,31-74$ & $8-10$ & 1.35 & TAG-1 & Massive pyrite breccia & Medium & Fragment of sandy pyrite & & 48.6 & 400 & 460 & $<5$ \\
\hline $2 \mathrm{~N}-1,0-42$ & $1-7$ & 5.70 & TAG-1 & Massive pyrite breccia & Medium & Massive pyrite & & 45.8 & 1100 & 427 & $<5$ \\
\hline \multicolumn{12}{|l|}{$158-957 \mathrm{G}-$} \\
\hline $1 \mathrm{~N}-1,0-40$ & $1-7$ & 12.20 & TAG-1 & Massive granular pyrite & Fine & Massive, colloform pyrite & & 49.1 & 4500 & 491 & $<5$ \\
\hline $2 \mathrm{~N}-1,0-12$ & $1-2$ & 16.50 & TAG-1 & Massive granular pyrite & Medium & Massive, fragmental pyrite & & 47.3 & 940 & 530 & $<5$ \\
\hline $3 \mathrm{~N}-1,0-30$ & 1 & 21.00 & TAG-1 & Pyrite-anhydrite breccia & Coarse & Clast or fragment & & 49.4 & 280 & 352 & $<5$ \\
\hline $3 \mathrm{~N}-1,30-77$ & $2-6$ & 21.30 & TAG-1 & Pyrite-anhydrite breccia & Fine & Clast or fragment & & 50.7 & 450 & 206 & $<5$ \\
\hline
\end{tabular}


Table 6 (continued).

\begin{tabular}{|c|c|c|c|c|c|c|c|c|c|c|c|c|c|c|}
\hline & $\begin{array}{l}\text { Element: } \\
\text { Method: }\end{array}$ & $\begin{array}{c}\text { As } \\
\text { INAA }\end{array}$ & $\begin{array}{c}\mathrm{Sb} \\
\text { INAA }\end{array}$ & $\begin{array}{c}\text { Mo } \\
\text { INAA }\end{array}$ & $\begin{array}{c}\text { Co } \\
\text { INAA }\end{array}$ & $\begin{array}{c}\mathrm{Se} \\
\text { INAA }\end{array}$ & $\begin{array}{c}\mathrm{Ni} \\
\text { INAA }\end{array}$ & $\begin{array}{c}\mathrm{Ca} \\
\text { INAA }\end{array}$ & $\begin{array}{c}\mathrm{Sr} \\
\text { INAA }\end{array}$ & $\begin{array}{c}\mathrm{Rb} \\
\text { INAA }\end{array}$ & $\begin{array}{c}\mathrm{Na} \\
\text { INAA }\end{array}$ & $\begin{array}{c}\mathrm{Br} \\
\text { INAA }\end{array}$ & $\begin{array}{c}\mathrm{U} \\
\text { INAA }\end{array}$ & $\begin{array}{c}\mathrm{Zn} \\
\text { INAA }\end{array}$ \\
\hline $\begin{array}{l}\text { Core, section, } \\
\text { interval }(\mathrm{cm})\end{array}$ & $\begin{array}{l}\text { Detection } \\
\text { limit: }\end{array}$ & $\begin{array}{c}2 \\
\mathrm{ppm}\end{array}$ & $\begin{array}{c}0.2 \\
\mathrm{ppm}\end{array}$ & $\begin{array}{c}5 \\
\mathrm{ppm}\end{array}$ & $\begin{array}{c}5 \\
\mathrm{ppm}\end{array}$ & $\begin{array}{c}5 \\
\mathrm{ppm}\end{array}$ & $\begin{array}{c}5 \\
\mathrm{ppm}\end{array}$ & $\begin{array}{c}50 \\
\mathrm{ppm}\end{array}$ & $1 \%$ & $\begin{array}{l}500 \\
\mathrm{ppm}\end{array}$ & $\begin{array}{c}30 \\
\mathrm{ppm}\end{array}$ & $\begin{array}{l}500 \\
\text { ppm }\end{array}$ & $\begin{array}{c}1 \\
\mathrm{ppm}\end{array}$ & $\begin{array}{c}0.5 \\
\mathrm{ppm}\end{array}$ \\
\hline $\begin{array}{l}15 \mathrm{R}-1,0-50 \\
16 \mathrm{R}-1,0-20 \\
17 \mathrm{R}-1,0-47 \\
18 \mathrm{R}-1,0-20\end{array}$ & & $\begin{array}{l}29 \\
46 \\
22 \\
52\end{array}$ & $\begin{array}{r}0.7 \\
0.8 \\
<0.2 \\
1.2\end{array}$ & $\begin{array}{r}120 \\
78 \\
35 \\
160\end{array}$ & $\begin{array}{l}710 \\
520 \\
820 \\
470\end{array}$ & $\begin{array}{r}130 \\
42 \\
110 \\
76\end{array}$ & $\begin{array}{r}60 \\
<50 \\
70 \\
70\end{array}$ & $\begin{array}{r}1 \\
<1 \\
<1 \\
3\end{array}$ & $\begin{array}{l}<500 \\
<500 \\
<500 \\
<500\end{array}$ & $\begin{array}{l}<30 \\
<30 \\
<30 \\
<30\end{array}$ & $\begin{array}{r}490 \\
380 \\
1500 \\
570\end{array}$ & $\begin{array}{l}5 \\
2 \\
2 \\
6\end{array}$ & $\begin{array}{r}<0.5 \\
3.3 \\
<0.5 \\
4.0\end{array}$ & $\begin{array}{l}<0.5 \\
<0.5 \\
<0.5 \\
<0.5\end{array}$ \\
\hline $\begin{array}{l}\text { 158-957F- } \\
1 \mathrm{~N}-1,31-74 \\
2 \mathrm{~N}-1,0-42\end{array}$ & & $\begin{array}{l}81 \\
62\end{array}$ & $\begin{array}{l}2.7 \\
2.5\end{array}$ & $\begin{array}{l}130 \\
110\end{array}$ & $\begin{array}{l}300 \\
370\end{array}$ & $\begin{array}{l}<5 \\
<5\end{array}$ & $\begin{array}{l}60 \\
60\end{array}$ & $\begin{array}{l}1 \\
1\end{array}$ & $\begin{array}{l}<500 \\
<500\end{array}$ & $\begin{array}{r}80 \\
<30\end{array}$ & $\begin{array}{l}440 \\
340\end{array}$ & $\begin{array}{l}5 \\
5\end{array}$ & $\begin{array}{l}30 \\
13\end{array}$ & $\begin{array}{l}<0.5 \\
<0.5\end{array}$ \\
\hline $\begin{array}{c}158-957 \mathrm{G}- \\
1 \mathrm{~N}-1,0-40 \\
2 \mathrm{~N}-1,0-12 \\
3 \mathrm{~N}-1,0-30 \\
3 \mathrm{~N}-1,30-77\end{array}$ & & $\begin{array}{l}48 \\
78 \\
72 \\
64\end{array}$ & $\begin{array}{l}2.9 \\
2.1 \\
1.7 \\
1.3\end{array}$ & $\begin{array}{r}110 \\
170 \\
110 \\
80\end{array}$ & $\begin{array}{r}34 \\
110 \\
180 \\
470\end{array}$ & $\begin{array}{l}<5 \\
<5 \\
<5 \\
15\end{array}$ & $\begin{array}{l}70 \\
50 \\
50 \\
60\end{array}$ & $\begin{array}{l}1 \\
1 \\
2 \\
1\end{array}$ & $\begin{array}{l}<500 \\
<500 \\
<500 \\
<500\end{array}$ & $\begin{array}{l}<30 \\
<30 \\
<30 \\
<30\end{array}$ & $\begin{array}{l}380 \\
710 \\
430 \\
460\end{array}$ & $\begin{array}{r}7 \\
6 \\
<1 \\
4\end{array}$ & $\begin{array}{l}<0.5 \\
21 \\
16 \\
4.7\end{array}$ & $\begin{array}{r}0.9 \\
<0.5 \\
<0.5 \\
<0.5\end{array}$ \\
\hline
\end{tabular}

